\title{
Atmospheric and emissivity corrections for ground-based thermography using 3D radiative transfer modelling
}

Article

Accepted Version

Creative Commons: Attribution-Noncommercial-No Derivative Works 4.0

Morrison, W., Yin, T., Lauret, N., Guilleux, J., Kotthaus, S., Gastellu-Etchegorry, J.-P., Norford, L. and Grimmond, S. (2020) Atmospheric and emissivity corrections for groundbased thermography using 3D radiative transfer modelling. Remote Sensing of Environment, 237. 111524. ISSN 00344257 doi: https://doi.org/10.1016/j.rse.2019.111524 Available at https://centaur.reading.ac.uk/88107/

It is advisable to refer to the publisher's version if you intend to cite from the work. See Guidance on citing.

Published version at: http://dx.doi.org/10.1016/j.rse.2019.111524

To link to this article DOI: http://dx.doi.org/10.1016/j.rse.2019.111524

Publisher: Elsevier

All outputs in CentAUR are protected by Intellectual Property Rights law, including copyright law. Copyright and IPR is retained by the creators or other copyright holders. Terms and conditions for use of this material are defined in the End User Agreement. 


\section{CentAUR}

Central Archive at the University of Reading

Reading's research outputs online 


\title{
Atmospheric and emissivity corrections for ground-based thermography using 3D radiative transfer modelling
}

William Morrison ${ }^{\mathrm{a}^{*}}$, Tiangang Yin ${ }^{\mathrm{b}}$, Nicolas Lauret ${ }^{\mathrm{c}}$, Jordan Guilleux ${ }^{\mathrm{c}}$, Simone Kotthaus ${ }^{\mathrm{a}, \mathrm{d}}$, Jean-Philippe Gastellu-Etchegorry ${ }^{\mathrm{c}}$, Leslie Norford ${ }^{\mathrm{e}}$, Sue Grimmond ${ }^{\mathrm{a}}$

\author{
${ }^{\text {a }}$ Department of Meteorology, University of Reading, Earley Gate, Reading, RG6 6BB, UK \\ ${ }^{\mathrm{b}}$ Earth System Science Interdisciplinary Center, University of Maryland, USA \\ ${ }^{\mathrm{c}}$ CESBIO, Toulouse University (CNRS, CNES, IRD, UPS), Toulouse, France \\ ${ }^{\mathrm{d}}$ Institut Pierre Simon Laplace (IPSL), Ecole Polytechnique, France \\ ${ }^{\mathrm{e}}$ Department of Architecture, Massachusetts Institute of Technology, Cambridge, MA, USA \\ *Corresponding authors: william.t.morrison@reading.ac.uk, c.s.grimmond@reading.ac.uk
}

\begin{abstract}
Methods to retrieve urban surface temperature $\left(T_{\mathrm{S}}\right)$ from remote sensing observations with sub-building scale resolution are developed using the Discrete Anisotropic Radiative Transfer (DART, Gastellu-Etchegorry, Grau and Lauret, 2012) model. Corrections account for the emission and absorption of radiation by air between the surface and instrument (atmospheric correction), and for the reflected longwave infrared (LWIR) radiation from non-black-body surfaces ("emissivity" correction) within a single modelling framework. The atmospheric correction a) can use horizontally and vertically variable distributions of atmosphere properties at high resolution $(<5 \mathrm{~m})$; b) is applied here with vertically extrapolated weather observations and MODTRAN atmosphere profiles; and c) is a solution to ray tracing and cross section (e.g. absorption) conflicts (e.g. cross section needs the path length but it is typically unavailable during ray tracing). The emissivity correction resolves the reflection of LWIR radiation as a series of scattering events at high spatial $(<1 \mathrm{~m})$ and angular $(\Delta \Omega \approx 0.02$ sr) resolution using a heterogeneous distribution of radiation leaving the urban surfaces. The method is applied to a novel network of seven ground-based cameras measuring LWIR radiation across a dense urban area (extent: $420 \mathrm{~m}$ x $420 \mathrm{~m}$ ) where a detailed 3-dimensional representation of the surface and vegetation geometry is used. Our unique observation set allows the method to be tested over a range of realistic conditions as there are variations in: path lengths, view angles, brightness temperatures, atmospheric conditions and observed surface geometry. For pixels with $250( \pm 10) \mathrm{m}$ path length the median $\left(5^{\text {th }}\right.$ and $95^{\text {th }}$ percentile) atmospheric correction magnitude is up to 4.5 (3.1 and 8.1) $\mathrm{K}$ at 10:10 on a mainly clear-sky day. The detailed surface geometry resolves camera pixel path lengths accurately, even with complex features such as sloped roofs.
\end{abstract}

The atmospheric correction method evaluation, with simultaneous "near" ( $\sim 15 \mathrm{~m})$ and "far" ( $\sim 155 \mathrm{~m})$ observations, has a mean absolute error of $0.39 \mathrm{~K}$. Using broadband approximations, the emissivity correction has clear diurnal variability, particularly when a cool and shaded surface (e.g. north facing) is irradiated by warmer (up to $17.0 \mathrm{~K}$ ) surfaces (e.g. south facing). Varying the material emissivity with bulk values common for dark building materials $(\varepsilon=0.89 \rightarrow 0.97)$ alters the corrected roof (south facing) surface temperatures by $\sim 3(1.5) \mathrm{K}$, and the corrected cooler north facing surfaces by less than $0.1 \mathrm{~K}$. Corrected observations, assuming a homogeneous radiation distribution from surfaces (analogous to a sky view factor correction), differ from a heterogeneous distribution by up to $0.25 \mathrm{~K}$. Our proposed correction provides more accurate $T_{\mathrm{s}}$ observations with improved uncertainty estimates. Potential applications include ground-truthing airborne or space-borne surface temperatures and evaluation of urban energy balance models.

\section{Introduction}

Development of sustainable cities, informed by weather and climate models, requires a clear understanding of how urban areas modify the surface energy balance (SEB). A key variable in the SEB is the surface temperature $T_{\mathrm{s}}$ (Porson et al., 2010), which is affected by surface morphology, material composition and human activities. $T_{\mathrm{s}}$ observations are hence valuable for the evaluation and improvement of urban SEB models (Grimmond et al., 2010). While longwave infrared (LWIR) remote sensing (RS) from space provides $T_{\mathrm{s}}$ observations for this purpose at increasing resolutions (Chrysoulakis et al., 2018), their biased view of the full three-dimensional (3D) surface (Voogt and Oke, 2003) and low temporal resolution means the complex spatio-temporal variations of $T_{\mathrm{s}}$ related to components of the SEB are not fully captured. Ground-based LWIR thermography, however, allows temporally continuous observations of individual facets (e.g. roof, wall) and sub-facets (e.g. material, shadowing) that make up the 3D urban form (Voogt and Oke, 1997; Morrison et al., 2018). These observations are crucial for understanding uncertainties of satellite derived $T_{\mathrm{s}}$ and have proven valuable as inputs to urban SEB models studies (e.g. Ghent et al., 2010) and for model evaluation (e.g. Krayenhoff and Voogt, 2007; Pigeon et al., 2008; Harshan et al., 2018).

To derive $T_{\mathrm{s}}$ from RS, a range of corrections are required. A LWIR camera may record a radiometrically calibrated brightness temperature $\left(T_{\mathrm{b}}^{\mathrm{cam}}\right)$ that differs from $T_{\mathrm{s}}$ because of radiation emitted or attenuated by the atmosphere between the surface and the sensor (atmospheric effects). Further, emissivity effects arise from LWIR radiation emitted by and reflected between non-blackbody (BB) surfaces. Ground-based LWIR RS in urban areas has unique challenges associated with these corrections. Satellite $T_{\mathrm{s}}$ retrieval procedures (e.g. Wan, 2014) are not directly applicable as urban geometry, materials and radiative exchanges are resolved at sub-building scales (rather than from within a mixed satellite pixel). Depending on the viewing geometry and sensor resolution, a similar issue affects airborne observations (e.g. Voogt and Grimmond, 2000; Lagouarde et al., 2010).

There are few studies with full $T_{\mathrm{s}}$ retrieval from observations at sub-building scales in complex urban areas. Ground-based cameras sensitive to LWIR in the atmosphere window $(\sim 8-14 \mu \mathrm{m})$ can underestimate atmospherically corrected $T_{\mathrm{s}}$ by more than $6 \mathrm{~K}$ for surface-camera path lengths ( $\mathrm{z}^{\text {path }}$ ) of $\sim 300 \mathrm{~m}$ in an urban setting (Meier et al., 2011). Ground-based RS with oblique view angles cause $\mathrm{z}^{\text {path }}$ and atmospheric effects to vary greatly. Corrections have treated $\mathrm{z}^{\text {path }}$ as constant (e.g. Yang and Li, 2009) or spatially variable (e.g. Meier et al., 2011; Hammerle et al., 2017).

While $\mathrm{z}^{\text {path }}$ primarily influences the atmospheric correction, to correct for reflected radiation from non-BB surfaces (hereafter referred to as the emissivity correction, following Adderley, Christen and Voogt, 2015), quantifying the material emissivity and reflected radiance across the observed surfaces is critical. Facet surface materials and emissivity can be highly variable (Kotthaus et al., 2014). Although urban geometry is an important influence on scattered radiation from the sky and canopy elements (Harman, Best and Belcher, 2004), spaceborne or airborne RS emissivity corrections often only consider material effects (e.g. Mitraka et al., 2012; Chrysoulakis et al., 2018). To account for multiple scattering of radiation within street canyons, the emissivity correction has been parameterised using the sky view factors (SVF) for both urban earth observation (EO) (Yang et al., 2015, 2016) and sub-building scale ground-based LWIR RS (Adderley, Christen and Voogt, 2015).

The current methods to retrieve sub-building scale $T_{\mathrm{s}}$ contain limitations. Meier et al.'s (2011) correction procedure considers only the atmospheric effect, with a sensor specific lookup table based on the MODTRAN radiative transfer (RT) model. MODTRAN is based on 1-D analytical computation of atmospheric contributions, i.e. the 3-D environment is unaccounted for. Adderley et al.'s (2015) emissivity correction simplifies the reflected radiation contribution by assuming isothermal radiation emission relative to the SVF of the target surface. No previous 
study has accounted for both the atmospheric and emissivity corrections within a single framework that explicitly resolves the related RT processes with flexibility in both instrument siting and number.

Anisotropic factors determining the LWIR irradiance across urban surfaces may be important for the description (and correction) of emissivity effects in RS observations. LWIR radiance of clear sky varies with zenith angle (Verseghy and Munro, 1989), material and shadow patterns cause variability in surface temperature (Voogt and Oke, 1997; Morrison et al., 2018), and materials may have anisotropic emissivity (Sobrino and Cuenca, 1999). Relatively little is known about the magnitude of the uncertainties associated with these effects. Beyond Adderley et al.'s (2015) emissivity correction procedure, ground-based studies that derive $T_{\mathrm{s}}$ use: (1) bulk approximations for surface emissivity and reflected radiation (Yang and Li, 2009); (2) nocturnal observations (e.g. Ghandehari, Emig and Aghamohamadnia, 2018) when radiation received from sky and buildings are more similar; or (3) in-situ measurements (e.g. thermocouples affixed to surfaces, e.g. Rotach et al., 2005; Offerle et al., 2007) with very limited spatial extent and portability. Given the complexity of within-canopy radiation scattering, many studies avoid obtaining $T_{\mathrm{s}}$ altogether by assuming BB characteristics (Voogt and Oke, 1997; Christen, Meier and Scherer, 2012) meaning observations are brightness temperatures $T_{\mathrm{b}}(\mathrm{K})$ rather than $T_{\mathrm{s}}$. However, the magnitude of the emissivity correction can be substantial (Jiménez-Muñoz and Sobrino, 2006; Chen et al., 2016) with effects on spatial thermal variations (Morrison et al., 2018).

Here the objective is to retrieve high-quality $T_{\mathrm{s}}$ at the sub-building scale from ground-based LWIR RS within a single modelling framework with both atmospheric and emissivity corrections. The approach developed is flexible, uses RT, and is applicable to any high-resolution ground-based thermography. The 3D RT modelling accounts for atmospheric and emissivity effects using recent enhancements of the Discrete Anisotropic Radiative Transfer (DART, Gastellu-Etchegorry et al., 2015) model. Now, DART has an atmosphere around complex terrain features (e.g. urban areas) which is high resolution (here $2.5 \mathrm{~m}$ ), 3D and uses easily modifiable MODTRAN gas and aerosol optical properties. The scattering of LW radiation for the emissivity correction has high angular resolution (here $\approx 0.02 \mathrm{sr}$ ) and, unlike radiosity models, can account for anisotropic scattering effects. No other high-resolution 3D sensor view and RT model (e.g. LESS, Qi et al., 2019) accounts for the atmosphere or LWIR surface emission and multiple scattering effects or describes temperature and optical properties of the surface and atmosphere at similar resolutions. This is the first study to exploit and evaluate these high-resolution RT capabilities of DART which are shown to be highly valuable for complex 3D terrain (e.g. urban areas). Through using these methods, new insights into LWIR radiation exchanges between surfaces at high spatial resolution $(<1 \mathrm{~m})$ are obtainable.

After the theoretical background for the retrieval of $T_{\mathrm{s}}$ is introduced (Section 2), the methods developed using DART (Section 3.1) are outlined, separated into the atmospheric (Section 3.2) and emissivity (Section 3.3) corrections. The developed methods are applied to LWIR cameras in a dense urban canopy characterised at a uniquely high level of detail (LOD) (Section 4). The atmospheric correction is evaluated using observations (Section 5.1), while the emissivity correction results are assessed using a sensitivity analysis (Section 5.2). Alternative and future ways the method can be applied are discussed (Section 6).

\section{Theoretical background to the corrections}

Atmospheric correction of RS observations is undertaken to remove the effects of the emitted and absorbed radiation by the air between the sensor and target (Sobrino, Coll and Caselles, 1991). The spectrally dependent path radiance $\left(L_{\lambda}{ }^{\text {atm }}\right)$ and transmittance of the atmosphere $\left(\Gamma_{\lambda}^{\text {atm }}\right)$ between a target surface and a RS instrument contribute to the at-sensor radiance. For pixel (x, y) of a LWIR camera, the at-camera band radiance $\left(L^{\mathrm{cam}}, \mathrm{W} \mathrm{m}^{-2} \mathrm{sr}^{-1}\right)$ is (Meier et al., 2011):

$$
L^{\mathrm{cam}}(\mathrm{x}, \mathrm{y})=\int_{\lambda_{1}}^{\lambda_{2}} \mathrm{~d} \lambda\left[B_{\lambda}\left(T_{\mathrm{s}}\right)(\mathrm{x}, \mathrm{y}) \cdot \Gamma_{\lambda}^{\mathrm{atm}}(\mathrm{x}, \mathrm{y})+L_{\lambda}^{\mathrm{atm}}(\mathrm{x}, \mathrm{y})\right] \cdot R_{\lambda}(\mathrm{x}, \mathrm{y})
$$

Eqn. 1

where $R_{\lambda}$ is the RS instrument normalised spectral response function and $B_{\lambda}\left(T_{\mathrm{s}}\right)$ is the BB Planck LW radiance $\left(\mathrm{W} \mathrm{m}^{-2} \mathrm{sr}^{-1} \mu \mathrm{m}^{-1}\right)$ that exits the surface.

Eqn. 1 assumes the target surface is a perfect emitter of BB radiation, whereas typically the spectral emissivity $(\varepsilon \lambda)$ is less than unity so that the radiance $L_{\lambda}\left(\mathrm{W} \mathrm{m}^{-2} \mathrm{sr}^{-1} \mu \mathrm{m}^{-1}\right)$ emitted by a body at temperature $T$ is less than the Planck BB radiance at the same temperature (Becker and ZhaoLiang Li, 1995):

$$
\varepsilon_{\lambda}=\frac{L_{\lambda}(T)}{B_{\lambda}(T)}
$$

Eqn. 2

The spectral radiance from an opaque, non-BB surface located on a horizontal plane detected by a theoretical LWIR camera pixel at wavelength $\lambda\left(L_{\lambda}{ }^{\mathrm{cam}}(\mathrm{x}, \mathrm{y}), \mathrm{W} \mathrm{m} \mathrm{m}^{-2} \mathrm{sr}^{-1} \mu \mathrm{m}^{-1}\right)$ is a combination of emitted and reflected radiation from the surface, after correction for any atmospheric effects. Assuming $\varepsilon_{\lambda}$ is isotropic, the surface temperature can be related to $L_{\lambda}{ }^{\mathrm{cam}}$ by:

$$
L_{\lambda}^{\operatorname{cam}}(\mathrm{x}, \mathrm{y})=\varepsilon_{\lambda} B_{\lambda}\left(T_{\mathrm{s}}\right)+\left(1-\varepsilon_{\lambda}\right) \frac{1}{\pi} E_{\lambda}
$$

with $E_{\lambda}\left(\mathrm{W} \mathrm{m}^{-2} \mu \mathrm{m}^{-1}\right)$ the downwelling, isotropic spectral irradiance from the sky. Rearranging Eqn. 3 gives $B_{\lambda}\left(T_{\mathrm{s}}\right)$ :

$$
B_{\lambda}\left(T_{\mathrm{s}}\right)=\frac{L_{\lambda}^{\mathrm{cam}}(\mathrm{x}, \mathrm{y})-\left(1-\varepsilon_{\lambda}\right) \frac{1}{\pi} E_{\lambda}}{\varepsilon_{\lambda}}
$$

which is related to $T_{\mathrm{s}}$ using the inverse of the Planck function $\left(B \lambda^{-1}\right)$ as (Jimenez-Munoz et al., 2009):

$$
T_{\mathrm{s}}=B_{\lambda}^{-1}\left[B_{\lambda}\left(T_{\mathrm{s}}\right)\right]=c_{2} /\left[\lambda \cdot \ln \left(\frac{\mathrm{c}_{1}}{\lambda^{5} \frac{L_{\lambda}^{\mathrm{cam}}(\mathrm{x}, \mathrm{y})-\left(1-\varepsilon_{\lambda}\right) \frac{1}{\pi} E_{\lambda}}{\varepsilon_{\lambda}}}+1\right)\right]
$$

with $\mathrm{c}_{1}=1.191042 \times 10^{9}\left(\mathrm{~W} \mathrm{~m}^{-2} \mathrm{sr}^{-1} \mu \mathrm{m}^{-1}\right)$ and $\mathrm{c}_{2}=1.4387770 \times 10^{4}(\mu \mathrm{m} \mathrm{K})$ the first and second radiation constants.

In urban areas, the 3D surface structure gives rise to LW irradiance contributions from other surfaces and a reduction of sky irradiance. For a given point within the urban canopy, the spectral irradiance $\left(E_{\lambda}, \mathrm{W} \mathrm{m}^{-2}\right)$ can be described as (Nunez, Eliasson and Lindgren, 2000): 


$$
E_{\lambda}=\int_{2 \pi} L_{\lambda}^{\mathrm{sky}}\left(\Omega_{\downarrow}\right) \cos \theta d \Omega+\int_{2 \pi} L_{\lambda}^{\mathrm{can}}\left(\Omega_{\downarrow}\right) \cos \theta d \Omega
$$

with $L \lambda^{\text {sky }}\left(\Omega_{\downarrow}\right)\left[L \lambda^{\text {can }}\left(\Omega_{\downarrow}\right)\right]$ the spectral radiance $\left(\mathrm{W} \mathrm{m}^{-2} \mathrm{sr}^{-1} \mu \mathrm{m}^{-1}\right)$ that originates from the sky [canopy] after any multiple scattering received by the surface from solid angle $d \Omega$ (sr), with $\theta$ the angle of incidence to the surface normal. $L_{\lambda}{ }^{\text {can }}\left(\Omega_{\downarrow}\right)$ varies with surface temperature and emissivity within the given solid angle. For wavelengths in the LWIR atmospheric window, where thermal RS instruments are typically sensitive to absorption, emission, and scattering of LW radiation, the air within the canopy surfaces (i.e. between buildings) can be neglected if the path lengths are short (determined by canyon geometry). $L_{\lambda}^{\text {sky }}\left(\Omega_{\downarrow}\right)$ varies with wavelength, the depth of precipitable water within the atmosphere and the portion of the sky seen (Verseghy and Munro, 1989). Critically, both $L_{\lambda}$ sky and $L_{\lambda}^{\text {can }}$ change due to any prior scattering of both diffuse and specular radiation.

\section{Methods}

The correction of LWIR RS observations for atmospheric (Section 3.2) and emissivity (Section 3.3) effects are outlined then applied to a central urban area (Section 4). The methods suit any ground-based or airborne sensor for atmospheric window $(7-14 \mu \mathrm{m})$ radiation and are applied here to LWIR camera observations. Morrison and Yin (2019) provides further examples and tutorials.

\subsection{DART radiative transfer and sensor view model}

The Discrete Anisotropic Radiative Transfer (DART) model (Gastellu-Etchegorry, Grau and Lauret, 2012) is used to determine $T_{\mathrm{s}}$ from LWIR camera observations. This state-of-the-art 3D RT model has tools to generate and import surface and atmosphere properties of realistic landscapes, as well as to simulate RT processes in the shortwave (e.g. Wu et al., 2018) and longwave (e.g. Wang et al., 2018) IR spectrum. DART has been evaluated for simple terrain (Sobrino et al., 2011). Recent updates (Grau and Gastellu-Etchegorry, 2013; Gastellu-Etchegorry et al., 2017) have good agreement between the atmosphere RT in DART and MODTRAN simulations at the top of the atmosphere. Using a discrete ordinate (DO) ray tracing approach, DART simulates 3D RT processes in both natural and urban landscapes. Individual rays are tracked along discrete directions within angular cones that sample the $4 \pi$ space so the radiation from both the sky and landscape can be accurately estimated (Yin et al., 2013; Yin, Lauret and Gastellu-Etchegorry, 2015a). DART's “forward-tracking” allows emitted radiation from any combination of surface, sun, and atmosphere, which is unavailable in other software (e.g. LESS, Qi et al., 2019). For a full description of the DART model see Gastellu-Etchegorry et al. (2015).

Unlike Meier et al.'s (2011) MODTRAN-based atmospheric correction approach that uses sensor-specific lookup tables (LUT) for each pixel distance, DART's DO and 3D ray-tracing is combined with its recently updated ability to describe both the atmosphere and any number of virtual sensors among the landscape elements. This update is evaluated here for the first time with ground-based observations. Optical and temperature properties of air between the surface and sensor can be described at high horizontal and vertical resolution $(<5 \mathrm{~m})$. An update to DART's sensor view model, first shown here, accepts hemispherical to narrow field of view (FOV) radiometers and frame cameras. The number of sensors used does not significantly alter the computation time of forward ray tracing. The sensor view model now allows the landscape to be viewed from any location and direction with any sensor type rather than only downward directions with orthographic (Sobrino et al., 2011) or frame camera (Yin, Lauret and Gastellu-Etchegorry, 2015b; Morrison et al., 2018) perspectives. The virtual sensors can be set up to exactly reproduce "real-world" observations with any sensor view perspective, geometry and spectral response functions. Modelled output images for the perspective of these sensors include the atmospheric transmittance and the surface thermal emission and scattering. Such a model-based setup allows for very fast adjustments of virtual camera settings without the need to create specific correction factors for a new viewing geometry.

Multiple scattering effects on emissivity corrections are simulated using DART at ground-based LWIR camera spatial resolutions $(<1 \mathrm{~m})$. The DO approach tracks individual rays within the model landscape in many directions to simulate multiple scattering affecting the radiation reflected from the urban canopy surfaces that is then detected by the LWIR cameras.

To correct observations, DART uses a "model world" (MW) extending beyond the observed surface area, with the following components:

1) A vector-based 3D surface model (digital surface model, DSM) with a voxelated vegetation distribution (of e.g. trees and shrubs). The DSM consists of a mesh of triangles.

2) A spatial distribution of surface temperature and materials to apply across the DSM and vegetation geometry.

3) A spatial distribution of atmospheric properties to prescribe to the air around the terrain.

4) The position, view angle, resolution and focal length (if applicable) of the RS observations to be corrected.

DART populates the volume occupied by the MW surface with a $3 \mathrm{D}$ array of voxels with a selected horizontal $(\Delta \mathrm{X}=\Delta \mathrm{Y})$ and vertical $(\Delta \mathrm{Z})$ resolution (Gastellu-Etchegorry, 2008). Each voxel manages the tracking of radiation for the media that occupies its space and stores optical properties (e.g. surface emissivity, extinction coefficient of air), temperatures (surface and air), and land cover properties (e.g. surface orientation and material). Here, the atmospheric correction (Section 3.2) and multiple scattering of LWIR radiation (emissivity correction, Section 3.3) are determined using DART and the MW (Figure 1). DART virtual sensors are chosen as frame (or "pinhole") cameras (hereafter "MW cameras") with any straight line of the MW surface projected as a straight line for the camera perspective (Hartley and Zisserman, 2004). The RT processes are simulated for the perspective of these LWIR RS instruments to facilitate correction of atmospheric and emissivity effects on the observations. 


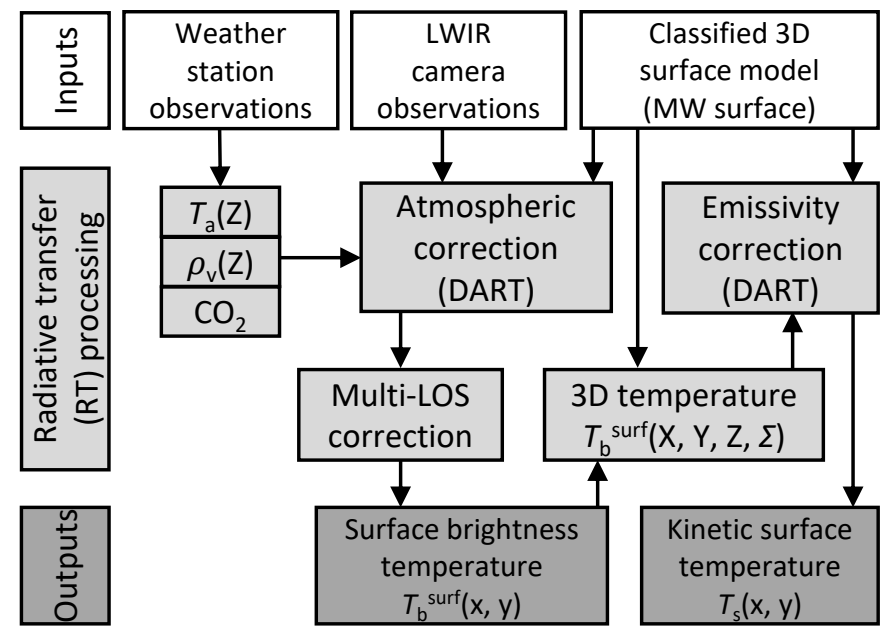

Figure 1. Procedure to correct longwave infrared camera observations for atmospheric and emissivity effects to obtain surface temperature ( $\left.T_{\mathrm{s}}\right)$ for each pixel. See list of symbols and acronyms for definitions.

\subsection{Atmospheric correction procedure \\ 3.2.1 DART simulation}

MW voxels that do not intersect any DSM geometry are "air voxels". Each air voxel contains aerosols and gases, with a respective cross section, density, single scattering albedo and scattering regime (Rayleigh function for gases, double Henyey-Greenstein functions for aerosols), air temperature $\left(T_{\mathrm{a}}, \mathrm{K}\right)$ and water vapour content. Horizontally, the gas and aerosol optical properties are homogeneous. A vertical profile is fitted with weather station observations based on an interpolation from MODTRAN gas and aerosol databases. The choice of voxel dimension needs to consider computational resources, MW surface complexity, sensor spatial resolution and surface-sensor path lengths. Following tests $\left(\mathrm{z}^{\text {path }}>\right.$ $100 \mathrm{~m}$ ) with the applied setup (Section 4) a voxel dimension of $<10 \mathrm{~m}$ was found to be suitable. There are no other studies at this high resolution for reference.

To simulate the atmosphere RT process in DART, all MW surfaces are initially prescribed a homogeneous kinetic surface temperature. Emission of rays $W$ from the surface ( $W^{\text {surf }}$ ) are used by DART to determine the path atmospheric transmittance. Rays that cross a DART camera pixel carry at-sensor spectral radiances for the atmosphere and surface components separately. $W^{\text {atm }}$ carry at-sensor spectral radiance from the atmosphere only $\left[L_{\lambda}^{\text {atm }}(\mathrm{x}, \mathrm{y})\right] . W^{\text {surf }}$ carry at-sensor radiance from the surface $\left[L_{\lambda}^{\text {cam }}\left(\mathrm{x}, \mathrm{y}, W^{\text {surf }}\right)\right]$ with $\Gamma_{\lambda}^{\text {atm }}(\mathrm{x}, \mathrm{y})$ determined by DART using (derived from Eqn. 1):

$$
\Gamma_{\lambda}^{\mathrm{atm}}(\mathrm{x}, \mathrm{y})=L_{\lambda}^{\mathrm{cam}}\left(\mathrm{x}, \mathrm{y}, W^{\text {surf }}\right) / B_{\lambda}\left(\mathrm{x}, \mathrm{y}, W^{\text {surf }}\right)
$$

Eqn. 7

By using BB surfaces and radiation tracked for one iteration, the transmittance is greater than if using non-BB surfaces and multiple scattering iterations. The benefit is shorter computation time and the overestimation is assumed to be negligible.

\subsubsection{Post-processing of DART simulation}

Although $\Gamma_{\lambda}^{\text {atm }}(\mathrm{x}, \mathrm{y})$ and $L_{\lambda}^{\text {atm }}(\mathrm{x}, \mathrm{y})$ can be used in the final atmospheric correction, with highly varying $\mathrm{z}^{\text {path }}$ across camera images a postprocessing step is implemented for the following reasons. Atmospheric transmittance and thermal emission between two points depends on the characteristics of the atmosphere (optical depth, single scattering albedo, temperature) present along that path. For LWIR the most important contribution comes from water $\left(\mathrm{H}_{2} \mathrm{O}\right)$ vapour, and to a lesser extent, carbon dioxide and ozone. The optical depth describes the spatial integral of the product of particle density and cross section. The cross section of $\mathrm{H}_{2} \mathrm{O}$ varies as a function of $\mathrm{z}^{\text {path }}$, relative humidity (RH, \%), $T_{\mathrm{a}}$, pressure and wavelength. With ray tracing, an essential conflict exists between the tracing of a ray and the variation of cross-section with $z^{\text {path }}$, whereby the $z^{\text {path }}$ and cross section calculation can only be determined after the ray tracing and associated RT calculations. Furthermore, within the thermal infrared spectral domain and across the bandwidth used $(0.1 \mu \mathrm{m})$, the ray transmission does not really follow Beer Lambert's law. As the cross section of $\mathrm{H}_{2} \mathrm{O}$ for the DART simulation cannot be calculated during ray tracing, the DART simulations (Section 3.2.1) use a single line of sight (SLOS, Meier et al., 2011) approximation where a single $\mathrm{H}_{2} \mathrm{O}$ cross section is applied to all air voxels. The average $\mathrm{z}^{\text {path }}$ of all camera pixels in the simulation ( $z^{\text {path,SLOS }}$ ) is used to derive the associated SLOS cross section. DART $\Gamma_{\lambda}^{\text {atm }}$ and $L \lambda^{\text {atm }}$ simulation outputs (Section 3.2.1) that use the cross sections based on $z^{\text {path,SLOS }}$ (outputs defined here as $\Gamma_{\lambda}^{\text {atm,SLOS }}$ and $L_{\lambda}{ }^{\text {atm,SLOS }}$ ) are then corrected for the multi-line of sight (MLOS) variation of $\mathrm{H}_{2} \mathrm{O}$ cross section to give $\Gamma_{\lambda}^{\text {atm }}$ and $L_{\lambda}^{\text {atm }}$ using a five-dimensional (5D) LUT, with dimensions: $\mathrm{z}^{\text {path }}(1 \mathrm{~m}-1000 \mathrm{~m}), \mathrm{RH}$ $(30 \%-100 \%), T_{\mathrm{a}}(259 \mathrm{~K}-315 \mathrm{~K})$, pressure $(880 \mathrm{hPa}-1050 \mathrm{hPa})$ and wavelength $(7 \mu \mathrm{m}-14 \mu \mathrm{m})$. The wavelength database has a spectral resolution of $0.1 \mu \mathrm{m}$. Therefore, it can handle any spectral response function of LWIR sensors unlike Meier et al. (2011) being limited to a specific sensor.

The LUT uses $z^{\text {path }}(x, y)$ and values from the same MODTRAN database as used by the SLOS approximation in the DART simulations. To obtain $z^{\text {path }}$, the MW instrumentation and 3D surface is loaded into rendering software (e.g. Blender, 2018) to render images from the MW instruments as $\mathrm{z}^{\text {path }}(\mathrm{x}, \mathrm{y})$ using the $\mathrm{z}$-buffer image channel output. This method is recommended as it requires less configuration compared to determining $\mathrm{z}^{\text {path }}(\mathrm{x}, \mathrm{y})$ by transforming 3D DSM geometry coordinates to the sensor view geometry (e.g. Meier et al., 2011).

The optical depths $(\tau)$ of $\mathrm{H}_{2} \mathrm{O}\left(\tau_{\lambda}{ }^{\mathrm{H} 20}\right)$ obtained from the 5D LUT for $\mathrm{z}^{\text {path,SLOS }}\left[\tau_{\lambda}{ }^{\mathrm{H} 20, \mathrm{SLOS}}(\mathrm{x}, \mathrm{y})\right]$ and $\mathrm{z}^{\text {path }}(\mathrm{x}, \mathrm{y})\left[\tau_{\lambda}^{\mathrm{H} 20, \mathrm{MLOS}}(\mathrm{x}, \mathrm{y})\right]$ are used to convert $\Gamma_{\lambda}^{\text {atm,SLOS }}(\mathrm{x}, \mathrm{y})$ to $\Gamma_{\lambda}^{\mathrm{atm}, \mathrm{MLOS}}(\mathrm{x}, \mathrm{y})$ using:

$$
\Gamma_{\lambda}^{\mathrm{atm}}(\mathrm{x}, \mathrm{y})=\exp \left[\ln \left(\Gamma_{\lambda}^{\mathrm{atm}, \mathrm{SLOS}}(\mathrm{x}, \mathrm{y})\right)+\tau_{\lambda}^{\mathrm{H} 2 \mathrm{O}, \mathrm{SLOS}}(\mathrm{x}, \mathrm{y})-\tau_{\lambda}^{\mathrm{H} 2 \mathrm{O}, \mathrm{MLOS}}(\mathrm{x}, \mathrm{y})\right] .
$$

For $L_{\lambda}^{\text {atm,MLOS }}$, the equivalent emissivity for the SLOS path $\left(\varepsilon_{\lambda}{ }^{\mathrm{SLOS}}\right)$ is estimated:

$$
\varepsilon_{\lambda}^{\mathrm{SLOS}}=L_{\lambda}^{\mathrm{atm}, \mathrm{SLOS}} / B_{\lambda}\left(\overline{T_{a}}\right)
$$


where $\overline{T_{a}}(\mathrm{~K})$ is the equivalent temperature of all air voxels in the MW area and $B_{\lambda}\left(\overline{T_{a}}\right)$ the Planck radiance at $\overline{T_{a}}$. The equivalent emissivity for each MLOS path $\left[\varepsilon_{\lambda}^{\mathrm{MLOS}}(\mathrm{x}, \mathrm{y})\right]$ is estimated with:

$$
\varepsilon_{\lambda}^{\mathrm{MLOS}}(\mathrm{x}, \mathrm{y})=1-\exp \left[\ln \left(1-\varepsilon_{\lambda}^{\mathrm{SLOS}}\right)+\tau_{\lambda}^{\mathrm{SLOS}}-\tau_{\lambda}^{\mathrm{MLOS}}(\mathrm{x}, \mathrm{y})\right]
$$

to calculate $L_{\lambda}^{\text {atm: }}$

$$
L_{\lambda}^{\mathrm{atm}}(\mathrm{x}, \mathrm{y})=\varepsilon_{\lambda}^{\mathrm{MLOS}}(\mathrm{x}, \mathrm{y}) \cdot B_{\lambda}\left(\bar{T}_{\mathrm{a}}\right) .
$$

This efficient (e.g. $<1$ min for six cameras each with 160 x 120 pixels) post-processing uses a Python script (Morrison and Yin, 2019) that can be configured by DART to run automatically after a main DART simulation. The post-processed $L_{\lambda}{ }^{\text {atm }}(\mathrm{x}, \mathrm{y})$ and $\Gamma_{\lambda}{ }^{\mathrm{atm}}(\mathrm{x}, \mathrm{y}) \operatorname{are}$ used to calculate the per-pixel and band-integrated LW radiance from the observed surface $\left[L^{\text {surf }}(\mathrm{x}, \mathrm{y}), \mathrm{W} \mathrm{m^{2 }} \mathrm{sr}^{-1}\right]$ using Eqn. $1 . L^{\text {surf }}$ is related to the surface brightness temperature $\left(T_{\mathrm{b}}{ }^{\text {surf }}\right)$ using a polynomial fit derived from a relation between band radiance and temperature, using band radiance calculated from:

$$
L=\int_{7 \mu \mathrm{m}}^{14 \mu \mathrm{m}} \mathrm{d} \lambda \cdot R_{\lambda}(\lambda) \cdot B_{\lambda}\left(T_{\mathrm{b}}\right)
$$

Eqn. 12

and fitted using a range of brightness temperatures $(250 \mathrm{~K} \rightarrow 350 \mathrm{~K}, \Delta \mathrm{K}=0.1)$.

\subsection{Emissivity correction procedure}

LW emission and scattering processes from surface reflected radiation is determined for the at-sensor radiance using DART multiple scattering simulations of LWIR radiation across the MW surface.

\subsubsection{Surface temperature and optical properties}

Optical properties and LWIR radiation exiting the MW canopy surfaces are assigned. DART voxels that occupy DSM geometry space are surface voxels $\left(\mathrm{Vx}^{S}\right)$ with a specified surface temperature and emissivity. Unlike other RT models with sub-facet resolution, geometry is not limited to planar 3D voxels (e.g. TUF, Krayenhoff and Voogt, 2007) nor are DSM triangles the smallest spatial unit (e.g. SOLENE, Hénon et al., 2012; Ghandehari, Emig and Aghamohamadnia, 2018). Thus, the DART combination of voxels and complex DSM geometry for RT models is both unique and highly flexible for prescribing and simulating surface properties for complex terrain. Here surface temperature and optical properties are assigned to voxels that occupy DSM surfaces classified by type (e.g. roofs, walls of different orientation, ground, grass).

Observed vegetation (e.g. trees or bushes) have leaves with optical properties as a turbid representation with a given angular distribution (Wang, $\mathrm{Li}$ and Su, 2007; Pisek, Ryu and Alikas, 2011). Given the highly heterogeneous urban surface: temperatures, materials, and RT processes; emissivity correction simulations are performed using a higher voxel resolution (i.e. $<2.5 \mathrm{~m}$ ) than the atmospheric correction.

\subsubsection{Simulation and emissivity correction}

DART-tracked rays are emitted across the surface geometry with varying surface temperatures and optical properties for the simulated wavelength(s) across the selected number of discrete directions $(\Omega)$ in the $4 \pi$ space. Each $\mathrm{Vx}^{S}$ face is split into multiple sub-faces to increase accuracy. Any rays tracked along the same discrete direction that cross the same sub-face are aggregated to a single ray.

A specified number of rays are emitted across the top layer of voxels in the MW (bottom of atmosphere (BOA) layer) to simulate the downwelling spectral radiance from the sky $\left(L_{\lambda}{ }^{\text {sky }}\right)$ using a prescribed isotropic sky brightness temperature $\left(T_{\mathrm{b}}{ }^{\text {sky }}\right)$. DART determines an isotropic $L_{\lambda}{ }^{\text {sky }}$ using the Planck function at the simulation wavelength.

After all rays are emitted and tracked to other surfaces or have crossed the BOA layer, some energy is scattered from the rays that intercept surface elements based on the surface reflectance $\left(1-\varepsilon_{\lambda}\right)$ under a state of thermodynamic equilibrium. Scattered rays are re-intercepted by surfaces for a specified maximum number of scattering events. A ray is halted if its energy becomes lower than a set threshold. Rays exiting a MW vertical side re-enter on the opposite side with the same direction but at a height that accounts for differences in topography between the exit and re-entry points.

Rays tracked across MW camera pixels determine the at-sensor spectral radiance from the surfaces as $L_{\lambda}{ }^{\text {DART }}(\mathrm{x}, \mathrm{y})\left(\mathrm{W} \mathrm{m} \mathrm{m}^{-2} \mathrm{sr}^{-1} \mu \mathrm{m}^{-1}\right)$. At each timestep both a BB $\left(\varepsilon_{\lambda}=1\right)$ and a non-BB $\left(\varepsilon_{\lambda}<1\right)$ DART simulation are processed to separate the radiation received by the surfaces within each camera pixel IFOV (instantaneous FOV). Both simulation types use the same voxel resolution (Section 3.3.1). DART allows for spatial variations in surface temperature unlike the SVF emissivity correction method (e.g. Adderley, Christen and Voogt, 2015).

MW camera images for the non-BB simulation $\left[L_{\lambda}{ }^{\mathrm{DART}}\left(\mathrm{x}, \mathrm{y}, \Omega_{\uparrow}, \varepsilon_{\lambda}<1\right)\left(\mathrm{W} \mathrm{m}^{-2} \mathrm{sr}^{-1} \mu \mathrm{m}^{-1}\right)\right]$ have radiance contributions from both emitted and reflected radiation leaving $\left(\Omega_{\uparrow}\right)$ the surfaces, which is analogous to $L_{\lambda}^{\text {cam }}$ (Eqn. 3). MW camera images for the BB simulation $\left[L_{\lambda}{ }^{\text {DART }}\left(\mathrm{x}, \mathrm{y}, \Omega_{\uparrow}, \varepsilon_{\lambda}\right.\right.$ $=1$ )] have radiance contributions from the emission only (analogous to $B_{\lambda}\left(T_{\mathrm{s}}\right)$, Eqn. 3). The BB simulation is computationally cheap as only the rays from surfaces within the FOV of the MW camera(s) are tracked. DART simulation results are used to separate the spectral radiance received $\left(\Omega_{\downarrow}\right)$ by the surfaces within the IFOV of each camera pixel $\left[L_{\lambda}{ }^{\text {DART }}\left(\mathrm{x}, \mathrm{y}, \Omega_{\downarrow}\right)\right]\left(\mathrm{W} \mathrm{m}^{-2} \mathrm{sr}^{-1} \mu \mathrm{m}^{-1}\right)$ by rearrangement of Eqn. 3 :

$$
L_{\lambda}^{\mathrm{DART}}\left(\mathrm{x}, \mathrm{y}, \Omega_{\downarrow}\right)=\frac{L_{\lambda}^{\mathrm{DART}}\left(\mathrm{x}, \mathrm{y}, \Omega_{\uparrow}, \varepsilon_{\lambda}<1\right)-\varepsilon_{\lambda}(\mathrm{x}, \mathrm{y}) L_{\lambda}^{\mathrm{DART}}\left(\mathrm{x}, \mathrm{y}, \Omega_{\uparrow}, \varepsilon_{\lambda}=1\right)}{1-\varepsilon_{\lambda}(\mathrm{x}, \mathrm{y})}
$$

with $\varepsilon_{\lambda}(\mathrm{x}, \mathrm{y})$ the per-pixel surface emissivity.

$\varepsilon_{\lambda}(\mathrm{x}, \mathrm{y})$ for Eqn. 13 can be created in two ways: a) the optical properties across the MW surface may have a simple or homogeneous distribution in the applied correction (Section 4) and have an isotropic scattering phase function (i.e. creating an $\varepsilon(\mathrm{x}, \mathrm{y})$ image mask); b) for scenes with more complex emissivity distributions including anisotropic scattering phase functions, the view angle dependent emissivity across an image can be determined using DART (Appendix A).

The final conversion of $T_{\mathrm{b}}{ }^{\text {surf }}(\mathrm{x}, \mathrm{y})$ to surface temperature $T_{\mathrm{s}}(\mathrm{x}, \mathrm{y})$ is performed using the inverse of the Planck function on the emissivity corrected spectral radiance:

$$
T_{\mathrm{s}}(\mathrm{x}, \mathrm{y})=B_{\lambda}^{-1}\left\{\frac{B_{\lambda}\left[T_{\mathrm{b}}^{\text {surf }}(\mathrm{x}, \mathrm{y})\right]-\left[1-\varepsilon_{\lambda}(\mathrm{x}, \mathrm{y})\right] L_{\lambda}^{\mathrm{DART}}\left(\mathrm{x}, \mathrm{y}, \Omega_{\downarrow}\right)}{\varepsilon_{\lambda}(\mathrm{x}, \mathrm{y})}\right\}
$$


using $L_{\lambda}{ }^{\mathrm{DART}}\left(\mathrm{x}, \mathrm{y}, \Omega_{\downarrow}\right)$ from Eqn. 13 to inform the at-sensor radiance contribution from scattered radiation. By simulation of RT processes at a narrow waveband, this approach assumes the surface and sky are grey bodies. Not explored in this study is the possibility of integrating over a broader range of wavelengths with spectral variance in surface emissivity.

\section{Application and Evaluation of Methods}

\subsection{Study area and observation sites}

The study area (Figure 2), in the Borough of Islington, London, UK (51³1'35” N, 006’19” W), has two primary observation sites on two highrise residential tower blocks: IMU at $74 \mathrm{~m}$ AGL (above ground level) and WCT at $36 \mathrm{~m}$ AGL. A third rooftop (CUB, $26 \mathrm{~m}$ AGL) is used for observational evaluation of the atmospheric correction. The area has an irregular arrangement of streets often lined with deciduous trees, with four- to six-storey residential and commercial buildings either as terraces or as large single units, parks with green space and asphalt, and three additional high-rise residential tower blocks (i.e. five high-rise buildings including IMU and WCT).
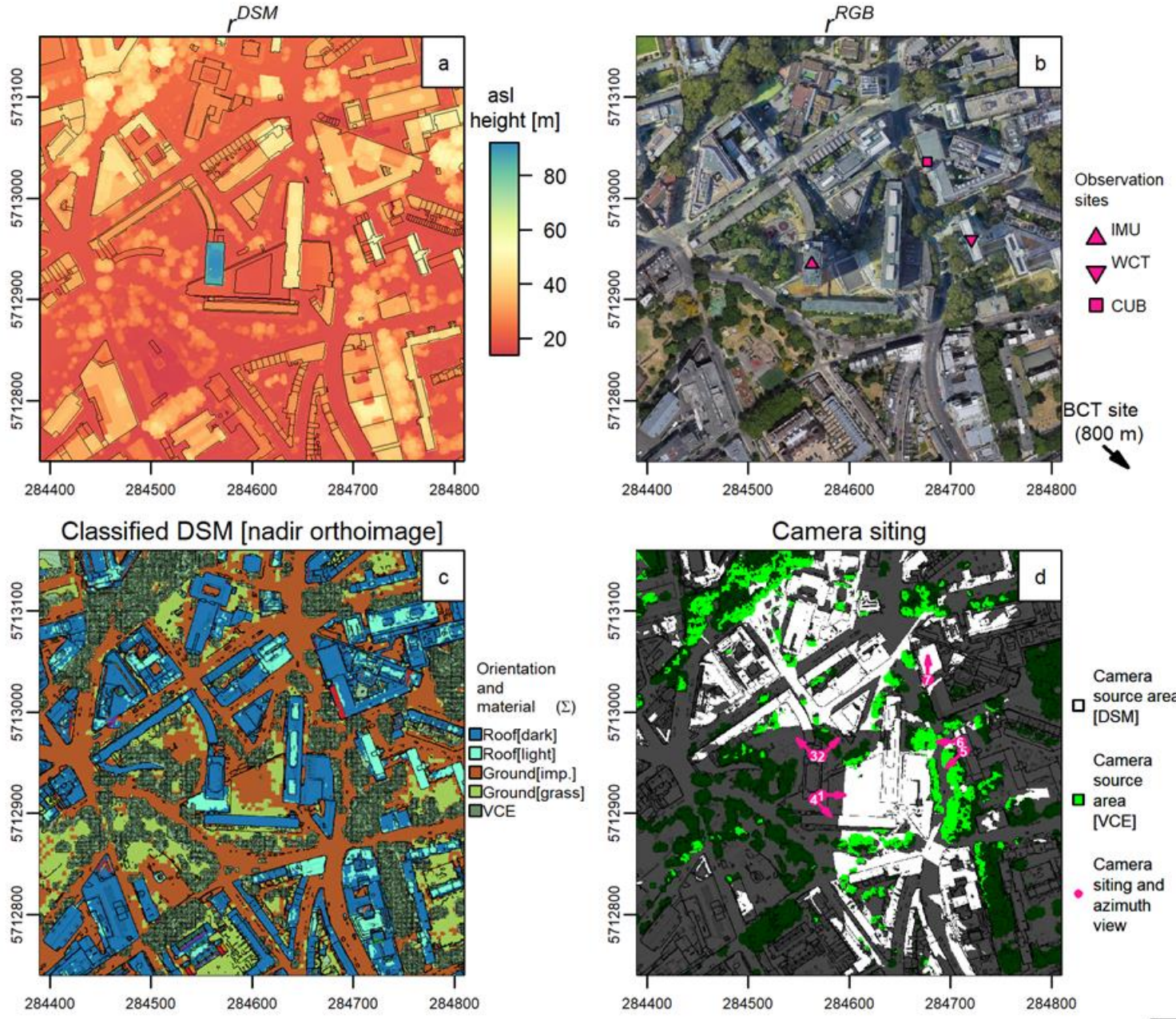

MW surface with MW camera perspective
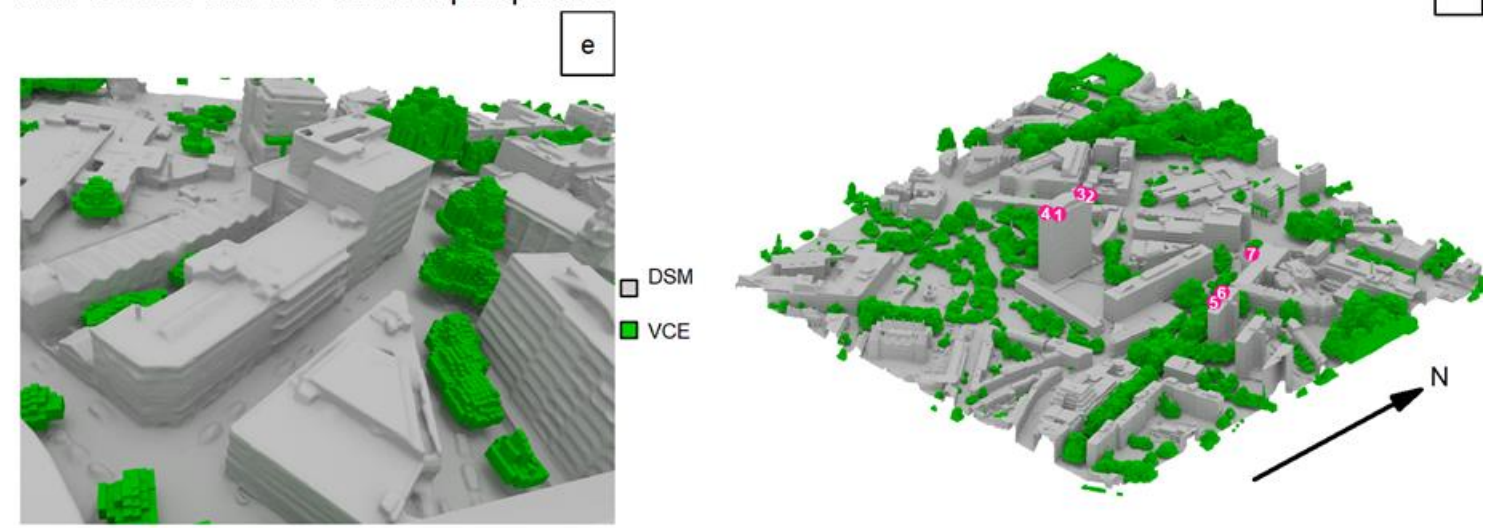

Figure 2. Study area characteristics: plan view of (a) above sea level (ASL) raster digital surface model ( $r^{D S M}$ ) of all surfaces with (black lines) a reference building footprint model from Evans, Hudson-Smith and Batty (2006), (b) orthorectified raster RGB $\left(r^{R G B}\right)$ image from a mosaic of Google Earth (Google, 2019a) images (Appendix B.1) with (symbols) locations of the study sites, (c) "model world" (MW) surface geometry with surface orientation and material properties $(\Sigma)$, (d) impervious and grass surfaces (white) and vegetation canopy elements (VCEs, light green) seen by the LWIR cameras within the observation network (

Table 1). Camera locations (numbers) shown as pink dots (white text) with approximate azimuthal facing (pink arrow). Dark colours are surfaces not seen by any camera. MW surface geometry rendered using Blender (Blender, 2018) for: (e) camera C2 perspective and (f) oblique orthogonal view of scene. Coordinates for $(\mathrm{a}-\mathrm{d})$ are Coordinate Reference System WGS84 UTM grid zone $31 \mathrm{~N}$ for study area extent of $420 \mathrm{~m}$ x $420 \mathrm{~m}$. 
The MW is a $420 \mathrm{~m}$ x $420 \mathrm{~m}$ plan area centred on the IMU site with a DSM and vegetation canopy elements (VCE) (Figure 2e, f) determined by Google Earth Pro (Google, 2019a) images and photogrammetry (Appendix B). The DSM (Figure 2e, f; grey) has $\sim 750,000$ triangles to capture all the Google Earth 3D surface elements except vegetation. VCE are a 3D array of voxels $\left(\mathrm{Vx}^{\mathrm{V}}\right)$ at $\Delta \mathrm{X}=\Delta \mathrm{Y}=1 \mathrm{~m}, \Delta \mathrm{Z}=0.1 \mathrm{~m}($ Figure $2 \mathrm{e}, \mathrm{f}$; green) where each voxel is either filled with or empty of VCE.

As with vegetation canopies (Kuusk, 2017), a key issue in developing 3D RT models for urban canopies relates to how the canopy structure is described. Here a uniquely high LOD canopy representation is created (Appendix B.1), with sub-facet structures (e.g. sloped roofs, balconies), rather than planar faces as used in other studies (e.g. Meier et al., 2011; Ghandehari, Emig and Aghamohamadnia, 2018). Its triangles are classified by orientation and material properties $(\Sigma)$ including cardinal facing, roofs, ground and vegetation (Figure 2c, Appendix B.2).

\subsection{Instrumentation and observations}

Optris PI-160 (Optris GmbH, 2018) LWIR cameras are deployed to observe the upwelling LWIR radiation (

Table 1; Figure 3). The small, lightweight, industrial-grade camera uses uncooled microbolometer detectors, with $25 \mu \mathrm{m} \times 25 \mu \mathrm{m}$ bolometer elements in a $160 \times 120$ focal plane array. The instrument outputs digital number (DN) values for each microbolometer pixel. DN values relate to at-sensor $7.5-14 \mu \mathrm{m}$ radiance and were radiometrically calibrated by the manufacturer two months prior to measurements using a $\mathrm{BB}$ reference. Each operational measurement is calibrated using an internal shutter with reference temperature and BB characteristics. During this calibration the shutter is put in the optical path of the instrument whereby its emission is sampled. The noise equivalent differential temperature (NEDT) is $0.1 \mathrm{~K}$ and the manufacturer's specified accuracy is $\pm 2 \mathrm{~K}$ at ambient temperatures $296 \pm 5 \mathrm{~K}$ (Optris GmbH, 2018). These specifications are typical of most microbolometer LWIR camera systems available and used for such applications (e.g. Meier and Scherer, 2012; Adderley, Christen and Voogt, 2015; Lee et al., 2018). The cameras have external enclosures (Figure 3, Appendix C and Supplement S1) to reduce body temperature changes from strong winds and/or direct sunlight and protect against corrosion. The application of the correction to these cameras does not include instrument uncertainty effects. The spectral response functions are derived in the laboratory (Supplement S2).
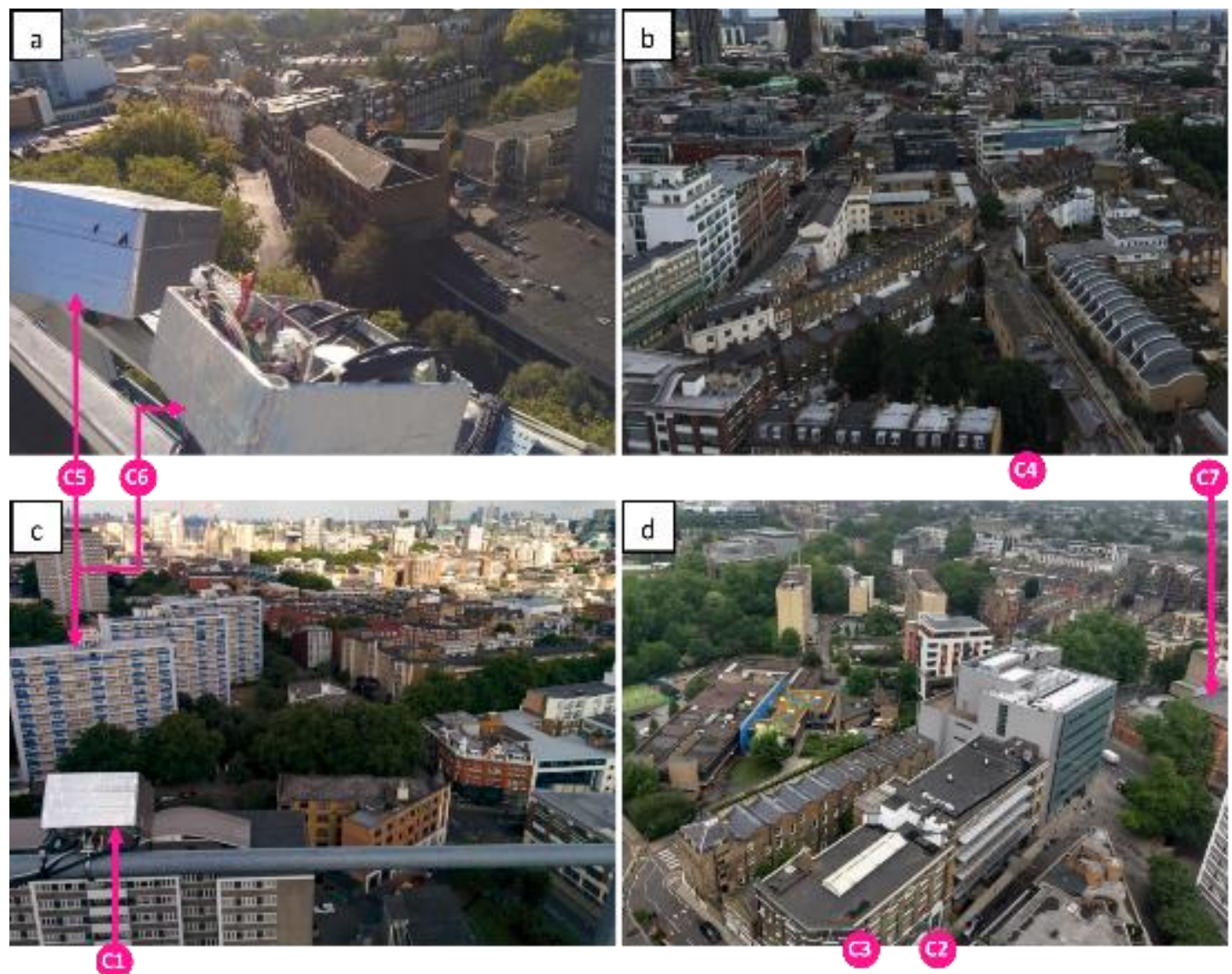

Figure 3. Digital camera images of: (a) cameras C5 and C6 taken at WCT site on 25 October 2017 looking southwest, with C6 enclosure shown open for maintenance, (b) southeast view from IMU site taken next to C4 on 21 July, (c) C1 enclosure taken at IMU site on 12 July looking east with WCT (C5,

C6) site in background, and (d) northeast view from IMU site taken next to C2 and C3 on 30 May, with a portion of the roof at CUB site sampled by C7 (Supplement S3) annotated.

Seven LWIR cameras installed at the IMU, WCT and CUB observation sites (Section 4.1) for the study period (7 July - 10 November 2017) enable sampling of multiple view angles of surfaces with different orientation, material, microscale structure and distances to the cameras.

Sample frequency is $1 \mathrm{~min}$ and a final temporal analysis is $5 \mathrm{~min}$ based on the median brightness temperature from 5 images (e.g. Figure $4 \mathrm{a}$ ). This is done to reduce the observational gaps.

Table 1. Siting properties of the ground-based longwave infrared (LWIR) cameras installed on high-rise residential towers (IMU, WCT; Figure 2, Figure

3) and City University of London Building roof (CUB, Supplement S3) within the study area. See list of symbols and acronyms for all other definitions.

\begin{tabular}{|c|c|c|c|c|c|c|c|}
\hline $\begin{array}{l}\text { Camera } \\
\text { name }\end{array}$ & $\begin{array}{l}\text { Installation } \\
\text { site }\end{array}$ & $\begin{array}{l}\text { Field of view }\left(^{\circ}\right) \\
\text { Horizontal } x \text { vertical }\end{array}$ & $\begin{array}{l}\text { Cardinal } \\
\text { facing }\end{array}$ & $\begin{array}{l}\text { Viewing zenith } \\
\text { angle }\left(^{\circ}\right)\end{array}$ & $\begin{array}{l}\text { 5th percentile } \\
\text { path length }(\mathrm{m})\end{array}$ & $\begin{array}{l}\text { Median path } \\
\text { length }(\mathrm{m})\end{array}$ & $\begin{array}{l}95 \text { th percentile } \\
\text { path length }(\mathrm{m})\end{array}$ \\
\hline C1 & IMU & FOV & $\mathrm{E}$ & $\theta$ & $\mathrm{z}^{\text {path }}$ & $\mathrm{z}^{\text {path }}$ & $\mathrm{z}^{\text {path }}$ \\
\hline $\mathrm{C} 2$ & IMU & $62.6 \times 49.1$ & $\mathrm{NE}$ & 51.7 & 70.1 & 97.9 & 198.93 \\
\hline $\mathrm{C} 3$ & IMU & $62.8 \times 49.2$ & NWW & 52.9 & 73.1 & 106.6 & 198.2 \\
\hline $\mathrm{C} 4$ & IMU & $37.3 \times 28.4$ & SE & 56.7 & 89.0 & 122.7 & 201.2 \\
\hline C5 & WCT & $38.4 \times 29.3$ & SW & 66.6 & 47.1 & 79.0 & 167.4 \\
\hline
\end{tabular}



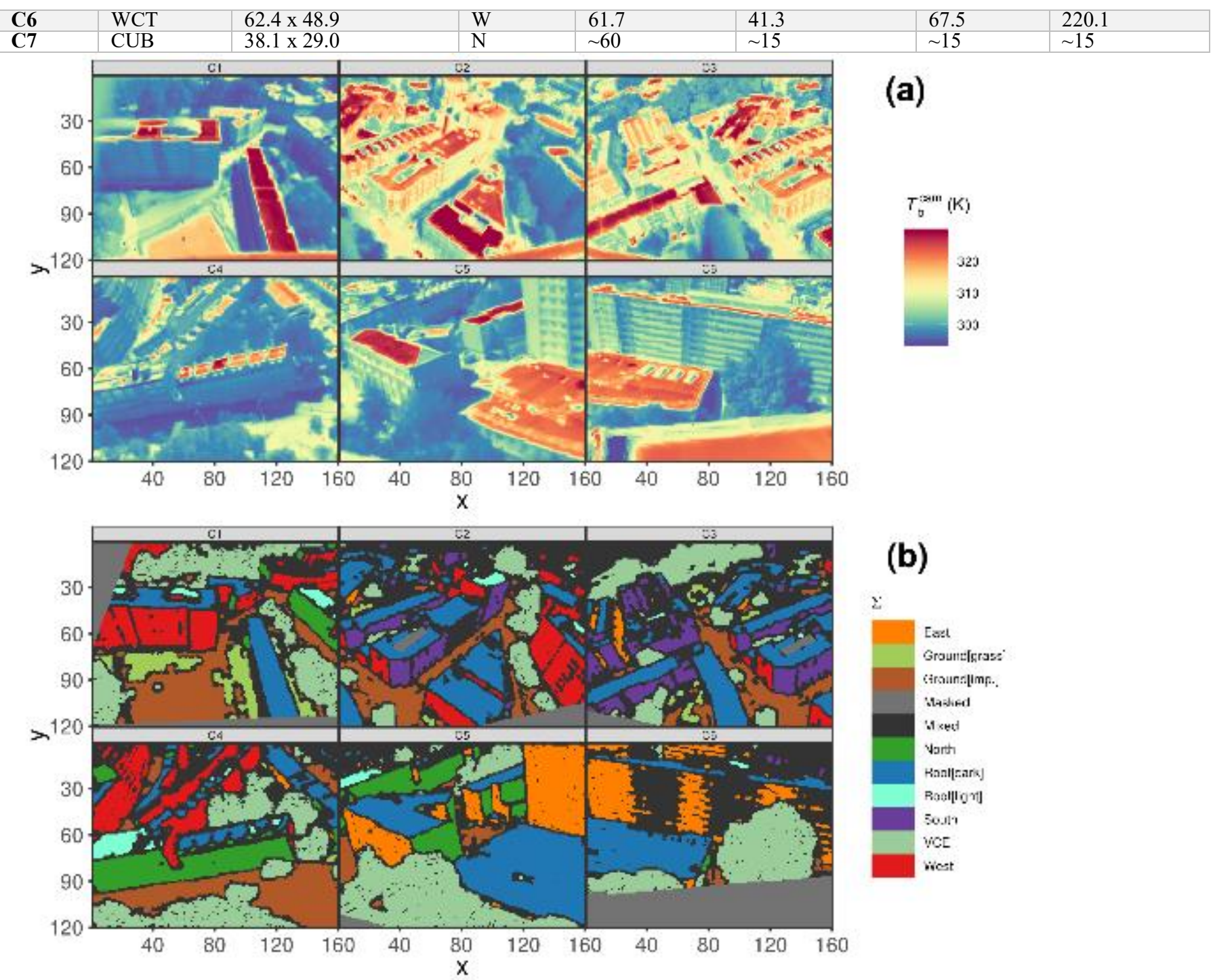

Figure 4. (a) Undistorted brightness temperature images ( $\left.T_{\mathrm{b}}{ }^{\text {cam }}\right)$ from Optris PI longwave infrared (LWIR) cameras at 11:30 on 27 August 2017 and (b) surface orientation and material class $(\Sigma)$ projected onto the image plane (IP) of each "model world" (MW) camera classified for each image pixel using the perspective projection (Morrison et al., 2018) of a similarly classified DSM (Appendix B.2).

The uncertainty in UTC time is assumed to be $<10 \mathrm{~s}$. The cameras require a $2 \mathrm{~h}$ "warm up" period (Morrison et al., 2018) to allow the currentinduced self-heating of the sensor elements to stabilize (Vollmer and Möllmann, 2017). Data prior to this are excluded. All data $0.5 \mathrm{~h}$ prior and 8 $\mathrm{h}$ after any rain event are excluded. A Davis Vantage Pro 2 weather station installed $114 \mathrm{~m}$ AGL on top of a residential tower block located at the BCT site (1.1 km southeast of the IMU site) provides measurements of rain rate $\left(\mathrm{mm} \mathrm{h}^{-1}\right)$ along with $T_{\mathrm{a}}, \mathrm{RH}$, and atmospheric pressure (hPa) required for the atmospheric correction routine. Similar to Adderley, Christen and Voogt (2015) a net radiometer (Kipp \& Zonen CNR1) installed at IMU next to $\mathrm{C} 4$ (Figure $2 \mathrm{e}, \mathrm{f})$ measured broadband $(4.5-42 \mu \mathrm{m})$ LWIR irradiance downward from the sky $\left(E_{\mathrm{LW}}{ }^{\text {sky }}, \mathrm{W} \mathrm{m}^{-2}\right)$ for the emissivity correction routine.

The LWIR camera lens distortion is corrected to match the rectilinear projection of the MW cameras. The MW cameras are sited in the model domain using on-site measurements of each camera location and view angle (

Table 1) and a fine-adjustment (Morrison et al., 2018).

The per-pixel orientation and material classes $(\Sigma(\mathrm{x}, \mathrm{y})$, Figure $4 \mathrm{~b})$ uses information from the similarly classified DSM (Appendix B.2) that is projected for the MW camera perspectives following methods by Morrison et al. (2018). $\Sigma(\mathrm{x}, \mathrm{y})$ enables the inter- $\Sigma$ classification of observations for use in prescribing temperature for the DART emissivity correction (Section 4.3.2).

\subsection{Model setup \\ 4.3.1 Atmospheric correction}

General model parameters for the atmospheric correction of observations are given in Table 2.

The vertical profile of gas and aerosols is informed by inputs of $T_{\mathrm{a}}$, RH and atmospheric pressure from the BCT measurements and the gas and aerosol databases are selected in DART (mid-latitude summer and urban $5 \mathrm{~km}$ visibility, respectively). As the weather station is $40 \mathrm{~m}$ above the top of the MW surface, $T_{\mathrm{a}}$ is extrapolated down to the bottom layer of the MW using the dry adiabatic lapse rate.

Given the large number of camera observations, DART simulations for each observation timestep ( 5 min) are not computationally viable ( 8 CPU threads per simulation: $\sim 12$ min using $\sim 8 \mathrm{~GB}$ memory and 8 processor cores). Instead, a 60 min simulation timestep is used. Post-processed (Section 3.2.2) results for each band and timestep are temporally interpolated to the observational resolution using a spline (Moritz and BartzBeielstein, 2017). Interpolated values near timesteps without observations (e.g. maintenance, quality control) are rejected. To reduce the number of emission sources and subsequent computation time, VCE geometry is excluded during atmospheric correction. 


\section{Emissivity correction}

For the downwelling LW radiation from the sky, $E_{\mathrm{LW}}{ }^{\text {sky }}$ from the CNR1 radiometer is related to $T_{\mathrm{b}}$ sky for DART (Table 2), using the StefanBoltzmann law:

$$
T_{\mathrm{b}}^{\mathrm{sky}}=\sqrt[4]{E_{\mathrm{LW}}^{\mathrm{sky}} / \sigma}
$$

The surface temperature across the MW (buildings, roads, etc) is approximated using atmospherically corrected surface brightness temperature $\left(T_{\mathrm{b}}{ }^{\text {surf }}\right)$ observations. The median $T_{\mathrm{b}}{ }^{\text {surf }}$ of all pixels within a certain orientation and material class $(\Sigma)\left\langle T_{\mathrm{b}}{ }^{\text {surf }}(\Sigma)\right\rangle($ class median $=\langle\rangle)$ is allocated to the associated DSM class (e.g. Figure 2c), except for VCE. As $T_{\mathrm{s}}$ for trees is nearly equal to $T_{\mathrm{a}}$ (Meier and Scherer, 2012), $T_{\mathrm{a}}$ from the Davis weather station is used for VCE. Broadband emissivity values are allocated for each DSM surface orientation and material class. As comprehensive spatial databases of urban emissivity are not readily available (Ghandehari, Emig and Aghamohamadnia, 2018), a representative range of urban emissivity values is used for the emissivity correction. Similar to Mitraka et al. (2012), the broadband ( $8-14 \mu \mathrm{m})$ emissivity from all non-metal and anthropogenic materials in a spectral library (SLUM, Kotthaus et al., 2014) is used as a fully opaque and grey body estimate for all non-vegetative surfaces (roof, ground, all walls) with the mean $\left(\varepsilon_{0.93}\right)$ considered as a baseline value. Minimum $\left(\varepsilon_{0.89}\right)$ and maximum ( $\left.\varepsilon_{0.97}\right)$ values are used in the sensitivity analysis (Section 5.2.3). VCE have a turbid representation of leaves within each $\mathrm{Vx}^{\mathrm{V}}$ and are given a spherical angular distribution. Leaves are given "deciduous leaf" optical properties at $10 \mu \mathrm{m}$ from the DART spectral database (leaf transmissivity $=0.0145$, reflectance $=0.0195)$ with a leaf area density [leaf area within voxel $/$ voxel volume $\left.\left(\mathrm{m}^{2} \mathrm{~m}^{-3}\right)\right]$ of $1.6(\mathrm{Lalic}$ and Mihailovic, 2004; Jeanjean et al., 2017).

Rays tracked from turbid VCE directly to camera pixels are not considered. Accurate tracking of rays from turbid media across camera pixels requires higher resolution DART runs (e.g. higher density of rays and voxel sub-faces) and/or leaves determined using the discrete triangle cloud option (Table 2). These factors are not tested as part of this study, so VCE pixel temperatures are not corrected for emissivity effects and are masked (e.g. Figure 4b). Ground-based thermography specific to urban trees and a simple emissivity correction applicable to observed VCE can be found in Meier and Scherer (2012).

Table 2. General model parameters set for the Discrete Anisotropic Radiative Transfer (DART) model atmospheric and emissivity correction routines.

\begin{tabular}{|c|c|c|c|}
\hline Model parameter & Units & Atmospheric correction & Emissivity Correction \\
\hline DART version used & - & 5.7 .4 build 1094 & 5.7.1 build 1058 (applicable: 5.7 .4 build 1094 ) \\
\hline Voxel dimension $\Delta \mathrm{X}, \Delta \mathrm{Y}, \Delta \mathrm{Z}$ & $\mathrm{m}$ & $2.5,2.5,2.5$ & $1,1,1$ \\
\hline Voxel sub-faces & - & 1 & 36 \\
\hline Wavelength(s) & $\mu \mathrm{m}$ & $7-14, \Delta \lambda=0.2$ & 10 \\
\hline Discrete directions & - & $\begin{array}{l}628 \text { (for image directions only, } \\
\text { see Yin et al., 2013) }\end{array}$ & 628 \\
\hline BOA ray density & rays $\mathrm{m}^{-2}$ & - & 1600 \\
\hline Surface ray density & rays $\mathrm{m}^{-2}$ & 10,000 & 10,000 \\
\hline Number of scattering events & - & 0 & 5 \\
\hline Surface temperature description & - & Homogeneous (300 K) & $\begin{array}{l}\text { Approximated using LWIR camera observations }\left(T_{\mathrm{b}}^{\text {surf }}\right) \text { classified by orientation } \\
\text { and material (e.g. Figure } 4 \mathrm{~b}) \text { prescribed across the classified DSM (e.g. Figure } \\
2 \mathrm{c}) \text {. See text for details. }\end{array}$ \\
\hline Emissivity & - & 1 & Bulk variation across anthropogenic surfaces using SLUM dataset $(0.89-0.97)$ \\
\hline
\end{tabular}

\section{Results \\ 5.1 Atmospheric correction}

To evaluate the atmospheric correction, two cameras (C2 and $\mathrm{C} 7$,

Table 1) both viewing a flat asphalt roof (C7 siting at CUB in Supplement S3) are used. C2 observes a $\sim 50 \mathrm{~m}^{2}$ area $\sim 155 \mathrm{~m}\left(\mathrm{z}^{\text {path }}\right)$ away which covers seven pixels (Figure $4 \mathrm{a}, \mathrm{x}=35, \mathrm{y}=140)$, whereas $\mathrm{C} 7\left(\mathrm{z}^{\text {path }}<15 \mathrm{~m}\right.$ ) has a 18542 pixel view. After DART shadow distribution simulations (Morrison et al., 2018), pixels are manually selected to exclude any shaded areas during the day as found in Meier et al.'s (2011) evaluation. Given the short C7 path length, atmospheric effects for this camera are assumed to be negligible [i.e. $\left.T_{\mathrm{b}}{ }^{\text {cam }}(\mathrm{C} 7) \approx T_{\mathrm{b}}{ }^{\text {surf }}(\mathrm{C} 7)\right]$.

During the predominantly cloudy multi-day (cf. to Meier et al.'s 2011 single-day) evaluation period (7 - 26 September 2017) the minimum (maximum) $T_{\mathrm{a}}$ is 281.4 (293.9) $\mathrm{K}$ and minimum (maximum) absolute humidity $\rho_{\mathrm{v}}$ was $7.03(12.71) \mathrm{g} \mathrm{m}^{-3}$ (Figure $5 \mathrm{c}$ ). Less cloudy daytime conditions near the end of the period (from 22 September) coincide with higher $T_{\mathrm{a}}$ values. The roof is fully sunlit (Figure $5 \mathrm{~d}$ ) from $\sim 40 \mathrm{~min}$ after sunrise (e.g. 15 September sunrise $=06: 30$, sample area fully sunlit at 07:05). Quality control (e.g. rain events (Figure 5d), camera maintenance) removed $1670(29 \%) 5$-min periods of $T_{\mathrm{b}}{ }^{\text {cam }}$ observations (Section 4.2).

The difference in the median brightness temperature observed by the two cameras $\Delta T_{\mathrm{b}} \mathrm{b}^{\text {cam }}=T_{\mathrm{b}}{ }^{\text {cam }}(\mathrm{C} 2)-T_{\mathrm{b}}^{\text {surf }}(\mathrm{C} 7)$ (Figure 5a, blue) quantifies the atmospheric effect on the raw observations. Generally, $\Delta T_{\mathrm{b}}{ }^{\mathrm{cam}}$ is negative during the day and approaches zero at night. As atmospheric absorption reduces the amount of radiation leaving the surface that is received by the sensor, the effect correlates with the absolute magnitude of surface temperature and is hence particularly strong during daytime when the surface is much warmer than the air. For clear and partly-cloudy daytime periods, $\Delta T_{\mathrm{b}}{ }_{\mathrm{b}}^{\text {cam }}$ is typically $<-2 \mathrm{~K}$ (minimum $-2.97 \mathrm{~K}, 15$ September $09: 15$ ) when the brightness - air temperature differences $\left(T_{\mathrm{b}}{ }^{\text {cam }}(\mathrm{C} 7)\right.$ $-T_{\mathrm{a}}$ ) are $>\sim 10 \mathrm{~K}$. Nocturnal clear and partly-cloudy periods (e.g. 12 and 16 September, Figure $5 \mathrm{~d}$ ) can have high $\Delta T_{\mathrm{b}}{ }^{\text {cam }}$ variability between timesteps. This variability may be explained by a combination of effects: intermittent cloud cover, anthropogenic heat sources, and potential differences of the sensor timesteps in the order of $\sim 10 \mathrm{~s}$ combined with fast temporal response (seconds) of $T_{\mathrm{s}}$ to changes in turbulent sensible heat fluxes (Christen, Meier and Scherer, 2012; Crawford et al., 2017).

After correction of atmospheric effects, $T_{\mathrm{b}}{ }^{\text {surf }}(\mathrm{C} 2)$ is significantly closer to the reference observations $\left(\Delta T_{\mathrm{b}}{ }^{\text {surf }}=T_{\mathrm{b}}{ }^{\text {surf }}(\mathrm{C} 7)-T_{\mathrm{b}}{ }^{\text {surf }}(\mathrm{C} 2)\right.$ Figure $5 \mathrm{a}$, black). The mean absolute error (MAE) between $T_{\mathrm{b}}^{\text {surf }}(\mathrm{C} 7)$ and $T_{\mathrm{b}}$ surf $(\mathrm{C} 2)$ is $0.39 \mathrm{~K}$ for all observations $\left(r^{2}=0.998\right.$, Figure 6$)$ and $0.48 \mathrm{~K}(0.28$ $\mathrm{K})$ for day (night) time observations, respectively. This is a significant improvement compared to the uncertainty associated with omitting the atmospheric correction particularly during daytime where $T_{\mathrm{b}}{ }^{\text {surf }}(\mathrm{C} 7)$ and $T_{\mathrm{b}}{ }^{\text {cam }}(\mathrm{C} 2)$ have $1.03 \mathrm{~K}$ MAE. While the magnitude of the atmospheric correction $\left(T_{\mathrm{b}}{ }^{\text {cam }}(\mathrm{C} 2)-T_{\mathrm{b}}\right.$ surf $(\mathrm{C} 2)$, Figure $5 \mathrm{a}$, red) generally follows the variations of atmospheric effect [quantified by $T_{\mathrm{b}}$ cam $(\mathrm{C} 2)-T_{\mathrm{b}}$ surf $(\mathrm{C} 7)$ ], some artefacts remain during morning when $\Delta T_{\mathrm{b}}$ surf is strongly positive at times. Of the $1^{\text {st }}$ percentile of $\Delta T_{\mathrm{b}}$ surf $(>1.02 \mathrm{~K})$, most $(93 \%, \mathrm{n}=39)$ occur between 07:00 - 09:00 under clear or partly-clear sky conditions. Observations with strongly negative $\Delta T_{\mathrm{b}}$ surf occur in the afternoon, with $95 \%(n=42)$ of observations in the $99^{\text {th }}$ percentile $\left(\Delta T_{\mathrm{b}}^{\text {surf }}<-0.97 \mathrm{~K}\right)$ being between 12:00 $-15: 00$. 
Both the strongly positive and negative $\Delta T_{\mathrm{b}}$ surf values could partly result from uncertainties in camera calibration. During morning, rapid changes in air temperature can cause uneven heating of the camera bodies. During the afternoon, the vertical profile of air temperature may cause the air temperature between the cameras to differ. As the dry adiabatic lapse rate rather than the environmental lapse rate are used, humidity is not accounted for (Section 3.2.1). Unfortunately, a weather station installed near ground level of the MW area to further inform vertical variation in temperature and water vapour for the correction and associated uncertainties failed shortly prior to the evaluation period.
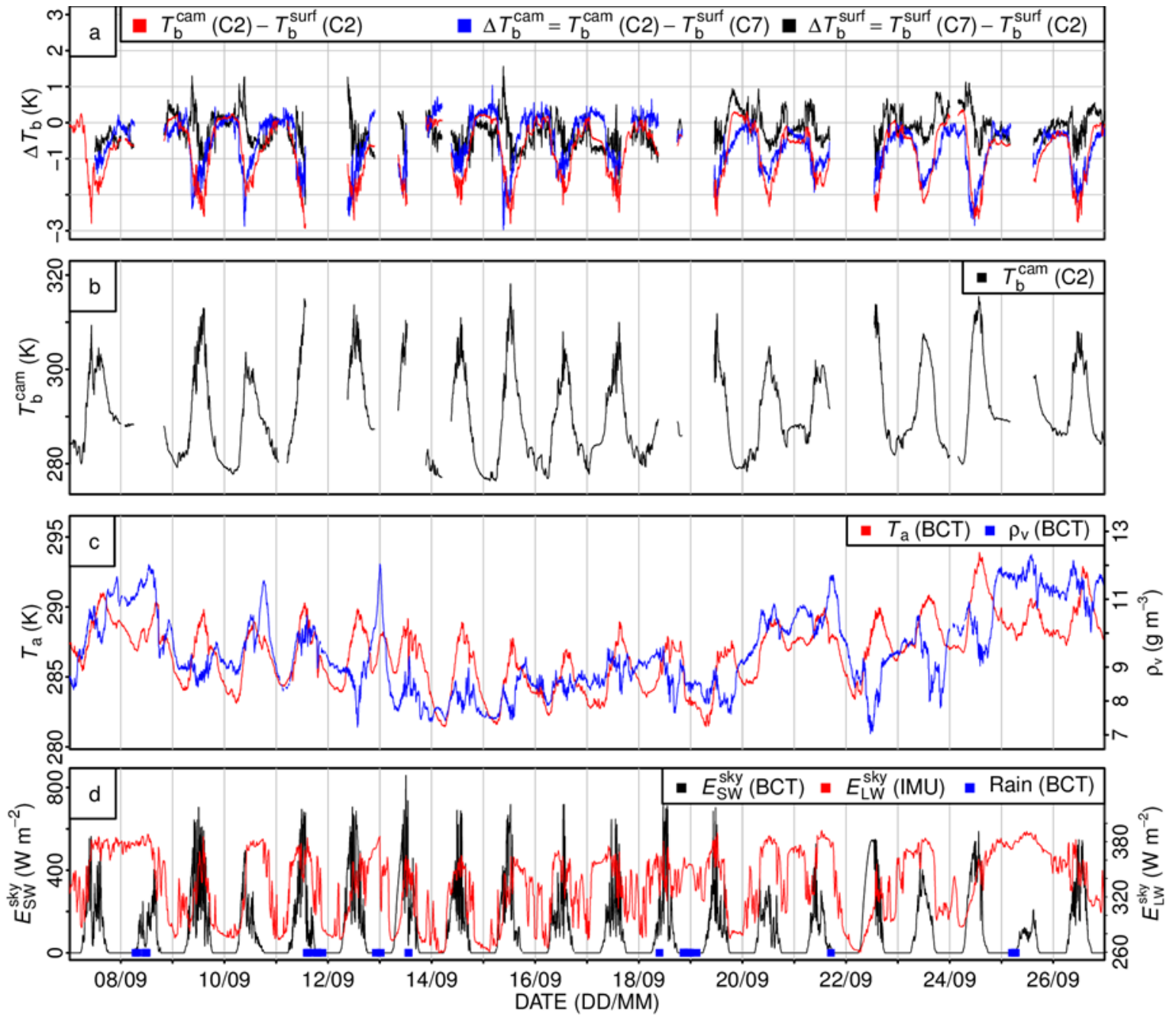

Figure 5. Atmospheric correction evaluation using the $\mathrm{C} 2$ and $\mathrm{C} 7$ cameras (

Table 1, Figure 2, Figure 3) and meteorological variables (Section 4.2) observed at BCT and IMU sites (Figure 2): (a) uncorrected ( $T_{\mathrm{b}}$ cam) minus corrected $\left(T_{\mathrm{b}}^{\text {surf }}\right)$ surface brightness temperature, (b) $T_{\mathrm{b}}{ }^{\text {cam }}(\mathrm{C} 2),(\mathrm{c})$ air temperature $\left(T_{\mathrm{a}}\right)$ and absolute humidity $\left(\rho_{\mathrm{v}}\right)$ and $(\mathrm{d})$ incoming shortwave $\left(E_{\mathrm{SW}}{ }^{\text {sky }}\right)$ and longwave $\left(E_{\mathrm{LW}}{ }^{\text {sky }}\right)$ radiation, and timing of rainfall (blue).

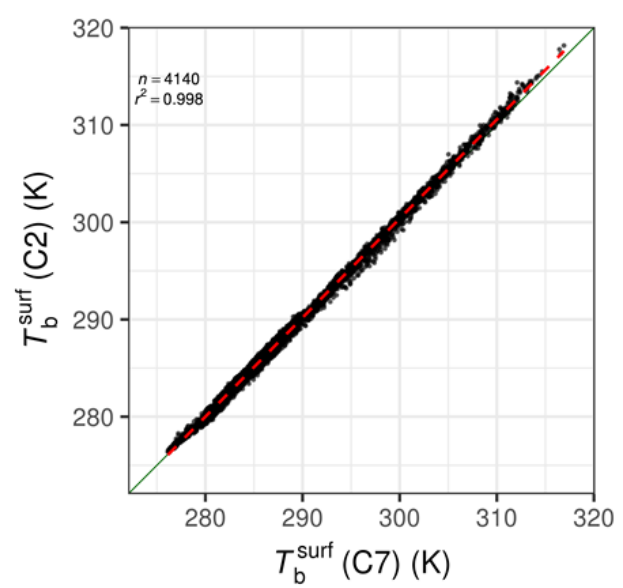

Figure 6. Comparison of surface brightness temperature corrected for atmospheric effects above a flat felt roof sampled with path length $\sim 155 \mathrm{~m}$ $\left[T_{\mathrm{b}}{ }^{\text {surf }}(\mathrm{C} 2)\right]$ and surface brightness temperature of the same surface sampled with path length $\sim 15 \mathrm{~m}\left[T_{\mathrm{b}}\right.$ surf $\left.(\mathrm{C} 7)\right]$ with negligible atmospheric effects.

When the atmospheric correction procedure is applied to all cameras with substantial path length differences (

Table 1) for the evaluation period, the greatest differences between the uncorrected at-sensor brightness temperature $T_{\mathrm{b}}{ }^{\text {cam }}(\mathrm{x}, \mathrm{y})$ to surface brightness temperature $T_{\mathrm{b}}{ }^{\text {surf }}(\mathrm{x}, \mathrm{y})$ are seen on 24 September. The median of $T_{\mathrm{b}}{ }^{\text {cam }}(\mathrm{x}, \mathrm{y})-T_{\mathrm{b}}$ surf $(\mathrm{x}, \mathrm{y})$ for pixels with $\mathrm{z}^{\text {path }}$ between $240-260 \mathrm{~m}$ reaches a minimum of $-4.53 \mathrm{~K}$ (largest absolute value) at 10:10. Impacts of different path lengths on this day are summarised in Figure 7 (Supplement S4 for other days). The variability of $T_{\mathrm{b}}{ }^{\text {cam }}(\mathrm{x}, \mathrm{y})-T_{\mathrm{b}}{ }^{\text {surf }}(\mathrm{x}, \mathrm{y})$ within each $\mathrm{z}^{\text {path }}$ bin (Figure 7) can be large (e.g. median $=-2.34 \mathrm{~K}$, interquartile range $(\mathrm{IQR})=1.93 \mathrm{~K}$ at $06: 00)$ as the magnitude of the correction varies based on the absolute value of $T_{\mathrm{b}}{ }^{\text {cam }}(\mathrm{x}, \mathrm{y})$, which can be highly variable in the urban setting (e.g. Figure 4a). 

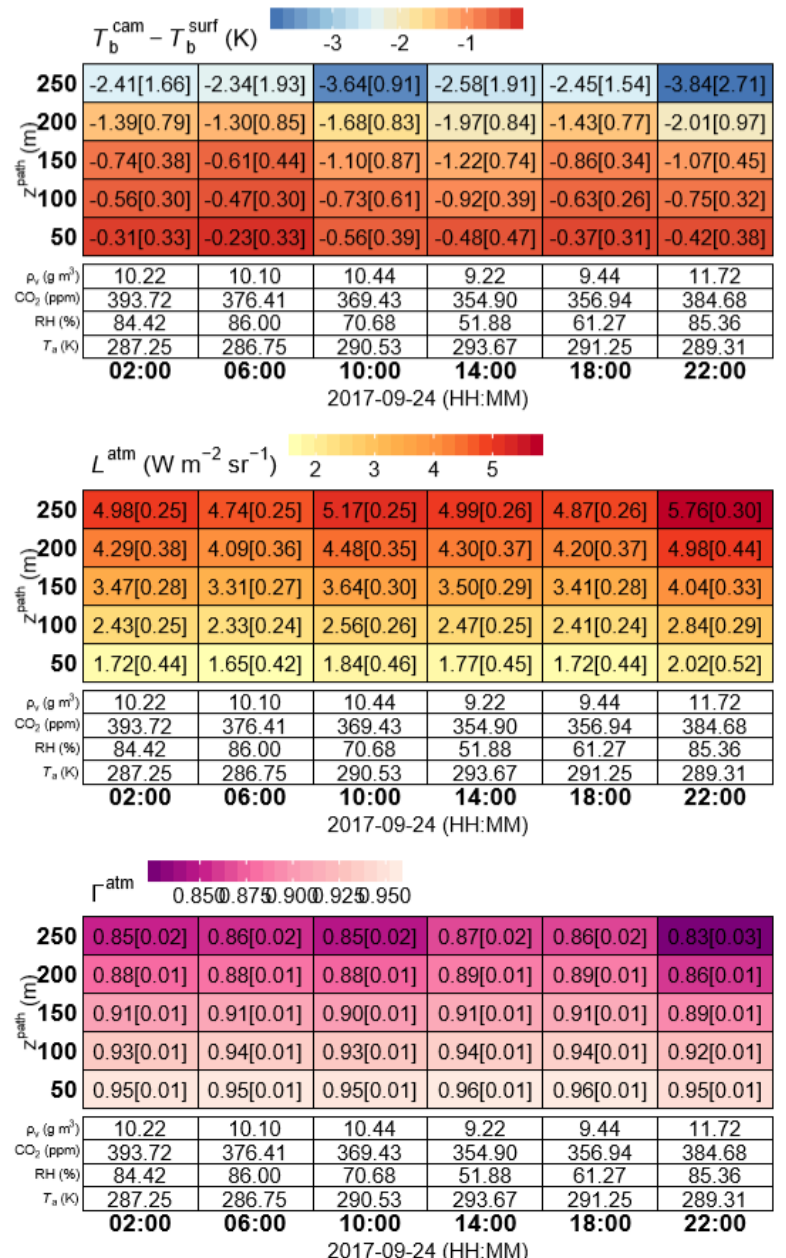

Figure 7. Atmospheric correction of longwave infrared (LWIR) camera observations for six timesteps on 24 September 2017, using pixels within $\pm 10 \mathrm{~m}$ of five path lengths ( $\mathrm{z}^{\text {path }}$ ) with (white cells) meteorological input variables (coloured cells) median[IQR] values of: (a) difference between uncorrected camera brightness temperature $\left(T_{\mathrm{b}}^{\mathrm{cam}}\right)$ and corrected surface brightness temperature $\left(T_{\mathrm{b}}\right.$ surf $)$, (b) surface-camera path contribution from the at-sensor band integrated atmosphere radiance $\left(L^{\mathrm{atm}}\right)$, and (c) surface-camera path contribution from band integrated atmospheric transmissivity $\left(\Gamma^{\mathrm{atm}}\right)$. See list of symbols and acronyms for all definitions and Supplement S4 for other days.

Analysis of all pixels from all cameras on 24 September (Figure 8a) indicates a decrease in the atmospheric effect $T_{\mathrm{b}}{ }^{\text {cam }}(\mathrm{x}, \mathrm{y})-T_{\mathrm{b}}{ }^{\text {surf }}(\mathrm{x}, \mathrm{y})$ with $^{\text {ith }}$ $z^{\text {path }}$. This is explained by the associated increase (decrease) of $L^{\mathrm{atm}}\left(\Gamma^{\mathrm{atm}}\right)$ (Figure $\left.8 \mathrm{~b}, \mathrm{c}\right)$. The outlier points (grey $<1000$ pixels or $0.003 \%$ of observations throughout the day) in Figure 8 are mainly from $\Gamma^{\mathrm{atm}}$ artefacts (Figure $9 \mathrm{c}$ ). The $1^{\text {st }}$ to $99^{\text {th }}$ percentile range in Figure $8 \mathrm{a}$ is -3.17 to $0.06 \mathrm{~K}$. These results are similar to Meier et al. (2011) who found $T_{\mathrm{b}}^{\text {cam }}-T_{\mathrm{b}}$ surf $\approx-6.5 \mathrm{~K}$ for $\mathrm{z}^{\text {path }}=310 \mathrm{~m}$ around midday, but are potentially underestimated compared to Adderley, Christen and Voogt (2015), where $T_{\mathrm{b}}$ cam $-T_{\mathrm{b}}{ }_{\mathrm{b}}^{\text {surf }}$ was up to $-8.6 \mathrm{~K}$ for a $15-75 \mathrm{~m} \mathrm{z}^{\text {path }}$ range. However, inter-study comparisons are challenging as differences in $\mathrm{z}^{\text {path }}, T_{\mathrm{b}}$ cam , meteorological conditions and spectral response functions affect the magnitude of atmospheric effects.
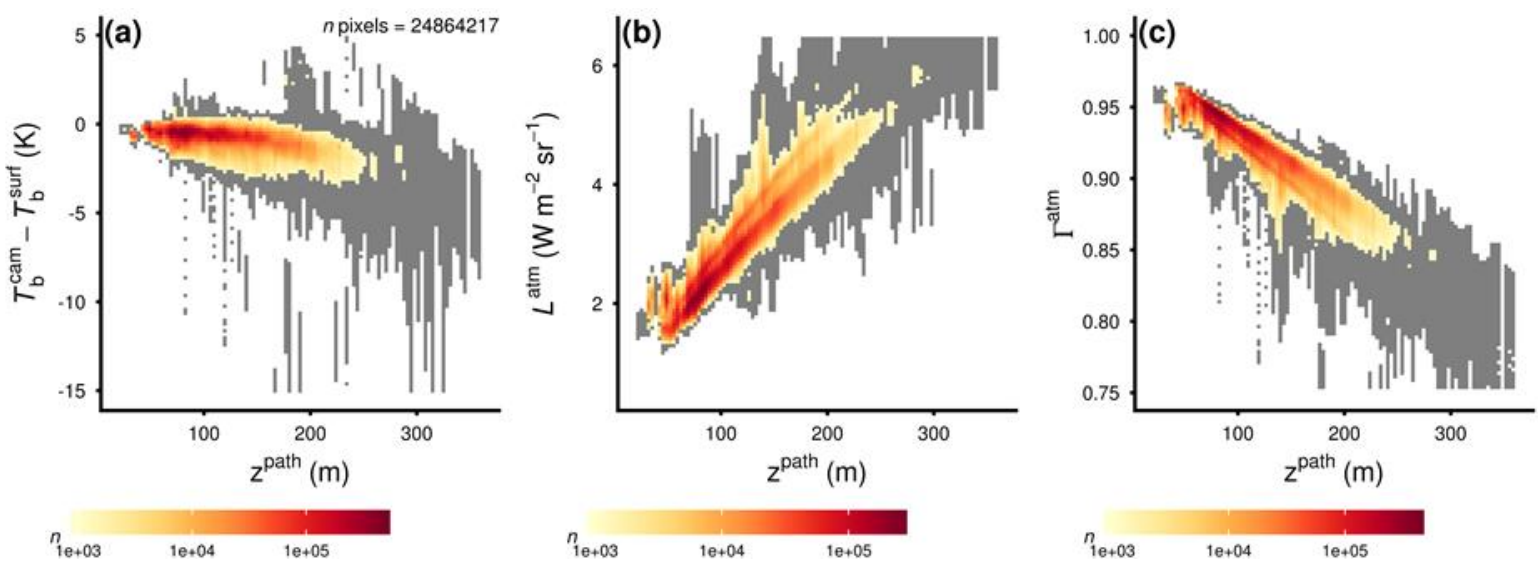

Figure 8. Per-pixel atmospheric correction of all longwave infrared (LWIR) camera observations at 5 min resolution on 24 September 2017 , with density of pixels (coloured shading; grey $<1000$ pixels) against surface-camera path length $\left(z^{\text {path }}\right)$ and (a) difference between uncorrected camera brightness temperature $\left(T_{\mathrm{b}}^{\mathrm{cam}}\right)$ and corrected surface brightness temperature $\left(T_{\mathrm{b}}^{\text {surf }}\right)$, (b) surface-camera path contribution of the at-sensor band integrated atmosphere radiance $\left(L^{\mathrm{atm}}\right)$, and (c) surface-camera path contribution of band integrated atmospheric transmissivity $\left(\Gamma^{\mathrm{atm}}\right)$.

Spatial variations of the atmospheric correction components ( $\mathrm{z}^{\text {path }}, L^{\mathrm{atm}}$ and $\left.\Gamma^{\mathrm{atm}}\right)$ for the study area are shown for the MW camera perspectives (Figure 9). As seen from the path lengths (Figure 9a), the complex real world (RW) surface geometry (Figure 2) is accurately reproduced, including buildings with complex footprints, multiple storeys (e.g. Figure 9a C3, $x=40, y=35$ ), and sloped roofs (e.g. Figure 9a C5, $x=40, y=$ $50 ; \mathrm{C} 6, \mathrm{x}=40, \mathrm{y}=10)$. Oblique view angles under RW conditions demand a high LOD surface geometry model, as simplified MW geometry (e.g. flat roofs, planar walls) could lead to inaccuracies in modelled surface-sensor view geometry. For example, a C6 pixel viewing a sloped roof (Figure 9a C6, $\mathrm{x}=40, \mathrm{y}=10$ ) with $\mathrm{z}^{\text {path }} \approx 75 \mathrm{~m}$ would not be registered to this roof when using a MW with flat roof low LOD geometry. This would lead to $\mathrm{z}^{\text {path }}>250 \mathrm{~m}$ and hence an error in atmospheric correction of over $3 \mathrm{~K}$ (Figure 7 ). 
Variability of $z^{\text {path }}$ with buildings and oblique viewing geometry (Figure 9a) is resolved by the atmospheric emission (Figure 9b) and transmissivity (Figure 9c) components of the correction. The spatial variability of $L^{\mathrm{atm}}(\mathrm{x}, \mathrm{y})$ and $\Gamma^{\mathrm{atm}}(\mathrm{x}, \mathrm{y})$ is related to the building geometry and $z^{\text {path }}$. Typically, a greater $z^{\text {path }}$ causes an increase (decrease) of $L^{\text {atm }}\left(\Gamma^{\mathrm{atm}}\right)$ (Figure 7). A small number of pixels underestimate $\Gamma^{\mathrm{atm}}$ (e.g. Figure $9 \mathrm{c}$ $\mathrm{C} 5, \mathrm{x}=65, \mathrm{y}=80$ ). For the surface within the IFOV of these pixels, the density of emitted rays ( $W^{\text {surf }}$, Section 3.2 .1 ) may be too low for the accurate determination of $L^{\mathrm{cam}}\left(W^{\text {surf }}\right)$. These artefacts can be eliminated by increasing the voxel resolution and the density of $W^{\text {surf }}$ at the expense of computation time. Pixels that view surfaces outside the MW area (e.g. Figure 9c C4, $x=38, y=5$ ) are excluded.

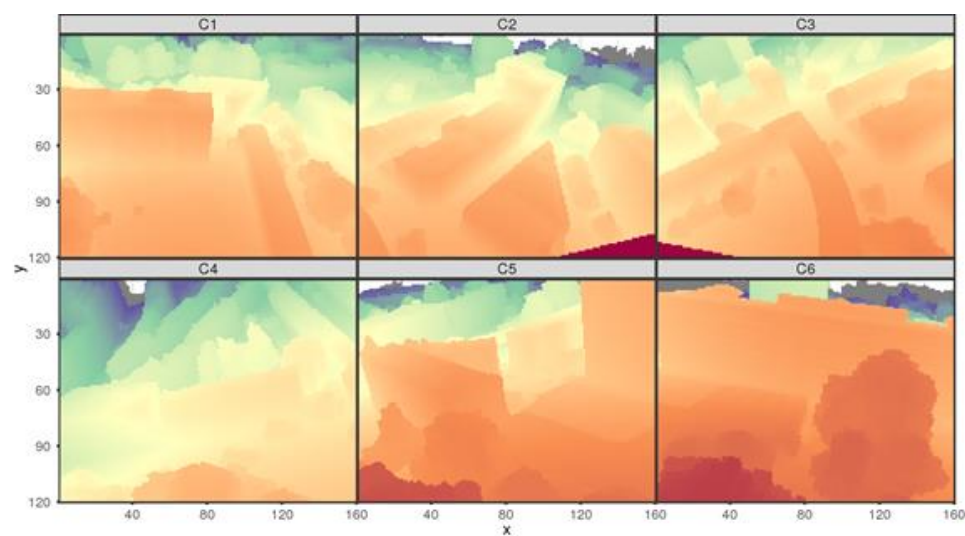

(a)

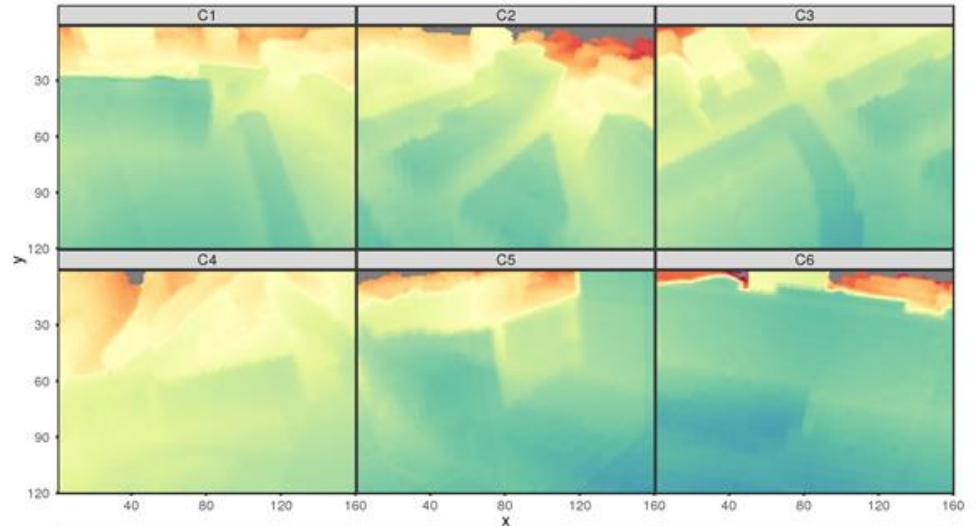

(b)

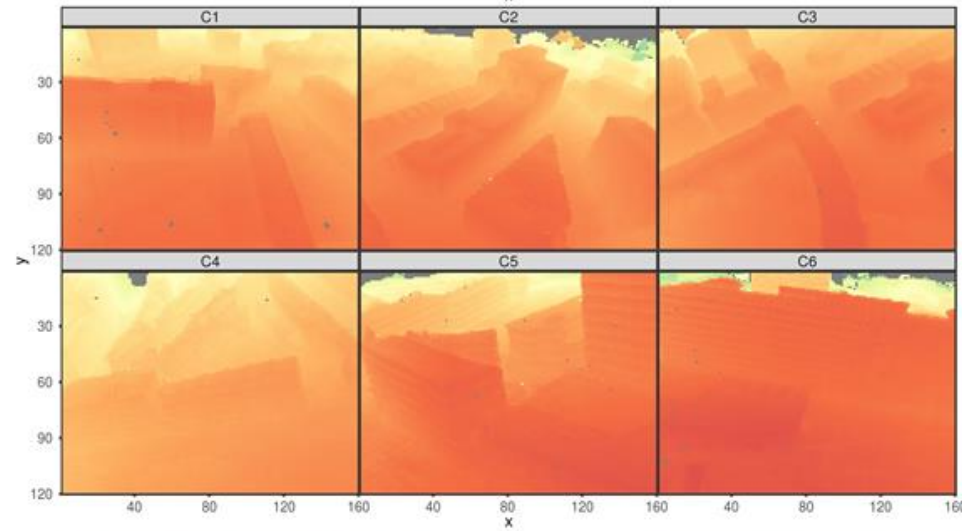

(c)
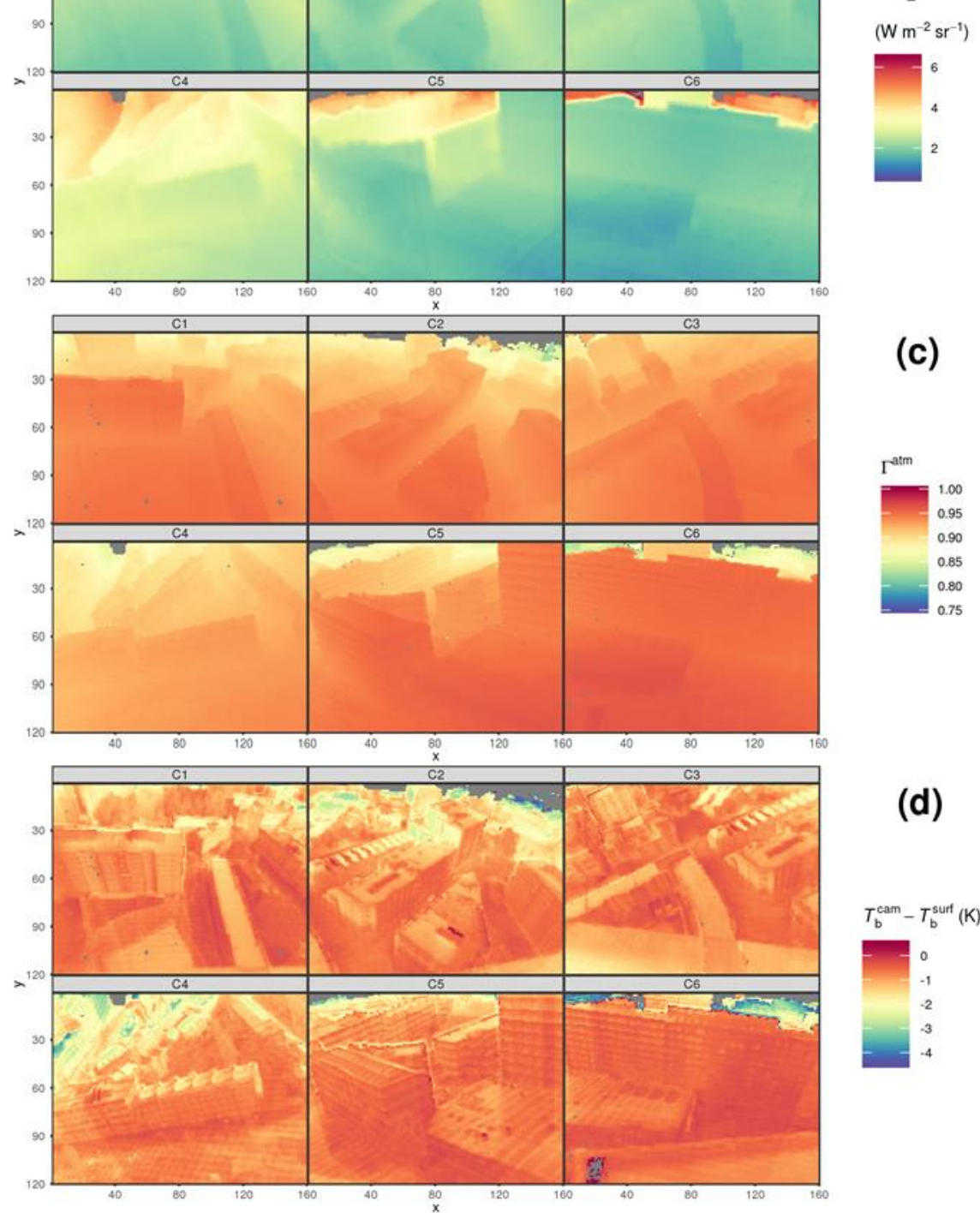

Figure 9. Atmospheric correction variables for each camera (

Table 1) at 12:00 (24 $4^{\text {th }}$ September 2017): (a) Surface - sensor path length ( $\left.\mathrm{z}^{\text {path }}, \mathrm{m}\right),(\mathrm{b})$ band integrated longwave emission from the atmosphere $\left[L^{\text {atm }}(\mathrm{x}, \mathrm{y})\right.$ $\left.=\int_{7 \mu \mathrm{m}}^{14 \mu \mathrm{m}} d \lambda \cdot \mathrm{R}_{\lambda}(\mathrm{x}, \mathrm{y}) \cdot L_{\lambda}^{\mathrm{atm}}(\mathrm{x}, \mathrm{y})\right]$ with $d \lambda=0.2$ and $\mathrm{R}_{\lambda}(\mathrm{x}, \mathrm{y})$ the sensor spectral response function, (c) band average atmospheric transmissivity. DART calculated $\Gamma_{\lambda}{ }^{\text {atm }}(\mathrm{x}, \mathrm{y})$ and $L_{\lambda}{ }^{\text {atm }}(\mathrm{x}, \mathrm{y})$, and (d) final difference between uncorrected $\left(T_{\mathrm{b}}{ }^{\text {cam }}\right)$ and corrected $\left(T_{\mathrm{b}}{ }^{\text {surf }}\right)$ brightness temperature observations. C3 shows more foreground roof than in Figure 4 (pixels excluded from all other results) as the view angle was altered between these dates. 


\subsection{Emissivity correction}

\subsubsection{Temporally resolved surface temperatures and incoming LWIR radiation}

The impact of the emissivity correction on the atmospherically corrected surface brightness temperatures $\left(T_{\mathrm{b}}\right.$ surf $)$ is assessed on a predominantly clear-sky day (27 August 2017, Figure 10) with large $T_{\mathrm{s}}$ variability between surfaces. For this analysis, the radiance received by the surface $\left[L_{\lambda}{ }^{\operatorname{DART}}\left(\mathrm{x}, \mathrm{y}, \Omega_{\downarrow}, \varepsilon_{0.93}\right)\right.$, Eqn. 13] is shown as broadband irradiance $E_{\mathrm{LW}}\left(\mathrm{W} \mathrm{m}^{-2}\right)$ using the Planck function and Stefan-Boltzmann law:

$$
E_{\mathrm{LW}}^{\mathrm{cam}}(\mathrm{x}, \mathrm{y})=\sigma B_{\lambda}^{-1}\left[L_{\lambda}^{\mathrm{DART}}\left(\mathrm{x}, \mathrm{y}, \Omega_{\downarrow}, \varepsilon_{0.93}\right)\right]^{4} .
$$

Surface brightness temperature observations used to prescribe spatial variability of radiation leaving the surfaces of different surface types $(\Sigma)$ differ broadly as a function of the incoming shortwave radiation $\left(E_{\mathrm{SW}}{ }^{\mathrm{sky}}\right)$ diurnal cycle (Figure $\left.10 \mathrm{~b}\right)$. While surfaces with a high SVF are more likely to receive shortwave energy input (Morrison et al., 2018), the inverse is true for longwave irradiance (Figure 10c) as surfaces within the canopy are warmer than the sky (for day and night). The median for north walls $\left\langle T_{\mathrm{b}}\right.$ surf $\left.\left(\Sigma_{\text {North }}\right)\right\rangle$ generally follows the diurnal cycle of $T_{\mathrm{a}}$ as the facets are mostly shaded throughout the day. $\left\langle T_{\mathrm{b}}^{\text {surf }}\left(\sum_{\text {East }}\right)\right\rangle$ peaks at 10:00 $(306.0 \mathrm{~K})$, while the maximum $(314.9 \mathrm{~K})$ of $\left\langle T_{\mathrm{b}}\right.$ surf $\left.^{\text {sin }}\left(\Sigma_{\text {south }}\right)\right\rangle$ is reached $\sim 100$ mins later. This relatively short time between maxima of east and south facing walls is explained by the predominant south-southeast facing direction of these facets (median azimuth for "South" wall is $147.9^{\circ}$, cf. for "East" is $91.6^{\circ}$ ).

Inter-class $T_{\mathrm{b}}{ }^{\text {surf }}$ variations contribute to the simulated differences in $E_{\mathrm{LW}}(\mathrm{x}, \mathrm{y}, \Sigma)$ (Figure 10c). This has implications on the final emissivity corrected observations (Figure 10d). Median differences for $E_{\mathrm{LW}}(\mathrm{x}, \mathrm{y}, \Sigma)$ reach $74.3 \mathrm{~W} \mathrm{~m}^{-2}$ between $\sum_{\text {Rooffdark] }}$ and $\Sigma_{\text {East }}$ during 13:00 - 14:55 (Figure 10c). The high SVF of roofs means $E_{\mathrm{LW}}$ for roof surfaces $\left[E_{\mathrm{LW}}\left(\mathrm{x}, \mathrm{y}, \Sigma_{\text {Roof }}\right)\right]$ are mostly composed of sky irradiance and hence is in closest agreement to the broadband radiometer observations used to specify $E_{\mathrm{LW}}{ }^{\mathrm{sky}}$, while east-facing walls receive large energy emissions from the opposing warm walls. The median for $E_{\mathrm{LW}}\left(\mathrm{x}, \mathrm{y}, \Sigma_{\text {Roof }}\right)$ is up to $19.9 \mathrm{~W} \mathrm{~m}^{-2}$ greater than the median $E_{\mathrm{LW}}{ }^{\text {sky }}$ during 13:00 - 14:55 (Figure 10c) as the roof receives some radiation from other surfaces. Inter-wall differences in the median of $E_{\mathrm{Lw}}(\mathrm{x}, \mathrm{y})$ reach $17.4 \mathrm{~W} \mathrm{~m}^{-2}$ between east and west walls during 13:00 - 14:55, which is driven by the lower temperatures of the shaded north-facing walls.

Of the walls, $E_{\text {Lw }}$ shows greatest variability for those facing east, which is explained by very small-scale variations of these structures. Cameras C5 and C6 primarily observe non-planar, east facing walls (Figure 4) with complex features such as balconies. Combined with the high zenith angle of observations (

Table 1), the cameras have a near-perpendicular view of the east walls and thus sample both the upper and lower parts of the balconies that have contrasting view factors to the sky and ground surfaces.
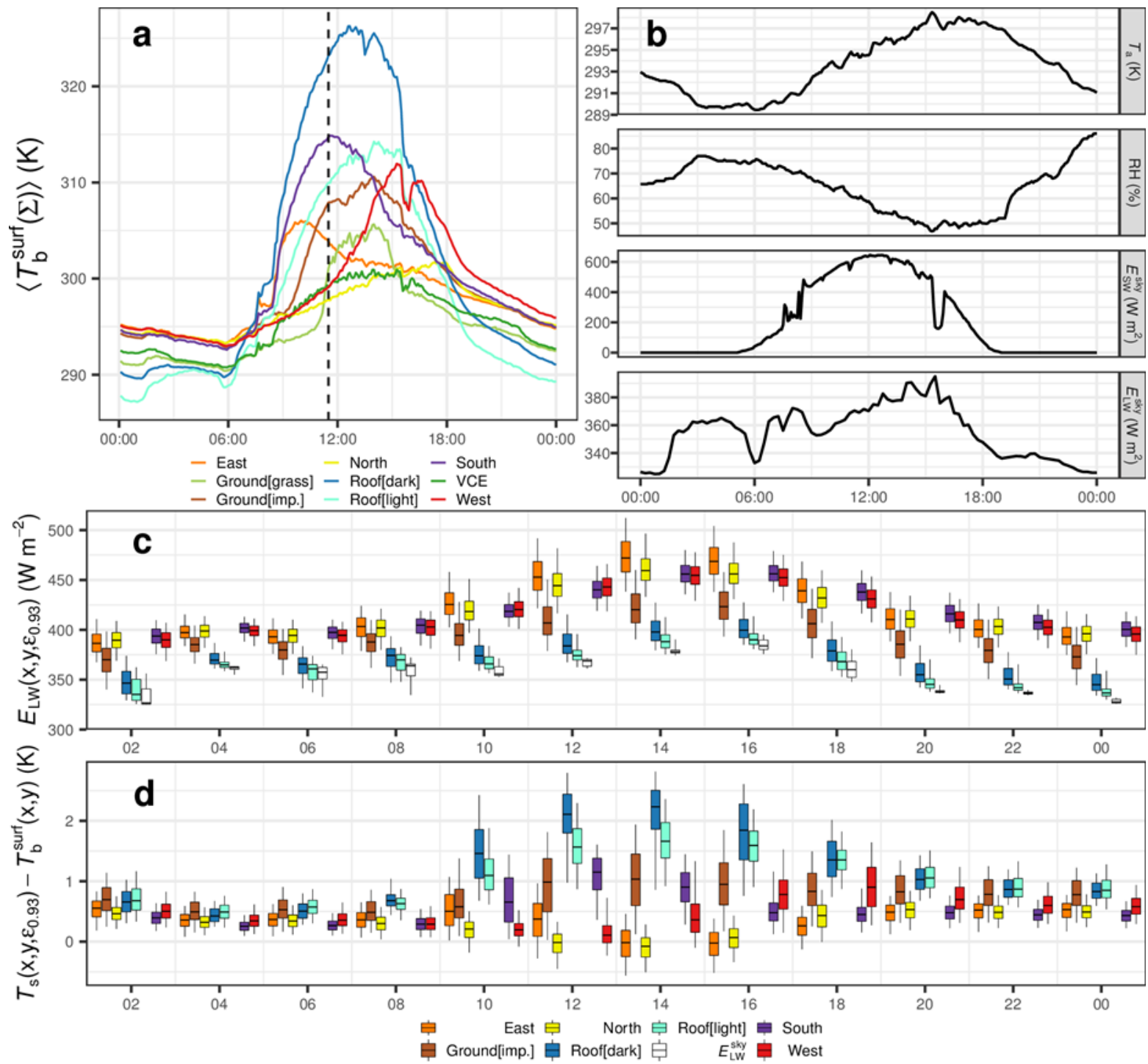

Figure 10. Observed and modelled data for 27 August 2017 stratified by surface orientation and material type ( $\Sigma$, colours) with (a) median per-pixel surface brightness temperatures $\left[T_{\mathrm{b}}^{\text {surf }}(\mathrm{x}, \mathrm{y})\right]$. For spatial pattern of emissivity correction (11:30, dashed lined) see Figure 11. (b) BCT observations of air temperature, relative humidity, Kipp \& Zonen CNR1 net radiometer broadband incoming shortwave $\left(E_{\mathrm{Sw}}{ }^{\mathrm{sky}}\right)$ and longwave $\left(E_{\mathrm{LW}}{ }^{\mathrm{sky}}\right)$ radiation. $(\mathrm{c})$ Broadband LWIR irradiance ( $\left.E_{\mathrm{LW}}\right)$ onto surfaces within the camera field of view, with $E_{\mathrm{LW}}$ sky for comparison. (d) Difference between emissivity (0.93) corrected surface temperature $\left[T_{\mathrm{s}}\left(\varepsilon_{0.93}\right)\right]$ for non-vegetative surfaces and surface brightness temperature. Boxplots: based on pixels from all camera images 
( 5 min resolution, for 2 h: 07:00 - 08:55 i.e. 08:00 is between 07:00 and 09:00 vertical lines) with $5^{\text {th }}$ and $95^{\text {th }}$ percentiles (whiskers), interquartile range (box), and median (horizontal line). $E_{\mathrm{LW}}{ }^{\text {sky }}$ boxplot uses 15 min resolution observations and min and max values (whiskers).

\subsubsection{Spatially resolved longwave irradiance and emissivity correction}

On 27 August 2017 the spatially resolved emissivity correction for all cameras (Figure 11, $T_{\mathrm{b}}$ cam in Figure 4a, Supplement S5 for combined atmospheric and emissivity correction) maximum inter-facet variability of surface brightness temperature occurs for within-canopy surfaces at 11:30 (Figure $10 \mathrm{a}$, dashed line). The $\left\langle T_{\mathrm{b}}{ }^{\text {surf }}\left(\Sigma_{\text {South }}\right)\right\rangle$ is $314.8 \mathrm{~K}$, or $17.0 \mathrm{~K}$ higher than $\left\langle T_{\mathrm{b}}{ }^{\text {surf }}\left(\Sigma_{\text {North }}\right)\right\rangle$. As a single surface emissivity is used for all the non-vegetative surfaces when correcting $T_{\mathrm{b}}^{\text {surf }}$, the magnitude of the correction (Figure 11b) is related to spatial differences in $E_{\mathrm{LW}}($ Figure 11a). The $E_{\mathrm{LW}}$ results account for RT process across the complex geometry seen by the RW camera observations (Figure $4 \mathrm{a}$ ), e.g. compare east wall balconies (C5 and C6), sloped roofs (e.g. C2 $x=40, y=40$ ), complex roofs (e.g. C1, $x=55, y=35 ; C 3, x=50 y=60 ; C 4 x \approx 70 \rightarrow 120, y$ $\approx 55 \rightarrow 70$ ), and vegetation (e.g. $C 2, x=120, y=80 ; C 6, x=120, y=75$ ). The atmospheric correction is not as sensitive to such small details across building facades.

Wall $E_{\mathrm{LW}}$ (Figure 11a) has high spatial variability associated with the wall geometry complexity. The overall $E_{\mathrm{LW}}$ increases closer to ground level and in narrow street canyons where SVF are reduced (Figure 11a). $E_{\mathrm{LW}}$ is typically lowest for roof surfaces (i.e. high sky view factor) and increasingly varies for roofs within the canopy (e.g. $\mathrm{C} 6 \mathrm{x}=40, \mathrm{y}=60$ ). Compared to adjoining walls, $E_{\mathrm{LW}}$ for ground surfaces is typically lower as there is a preferential orientation of ground surfaces to the cool sky. Overall, $E_{\mathrm{LW}}$ for the ground surfaces decreases with distance to buildings and is greater for ground surfaces close to trees, as these occlude the ground from most downwelling sky irradiance. When the longwave irradiance approaches the radiation emitted by a surface, the emissivity correction is minimised (Figure 10b).

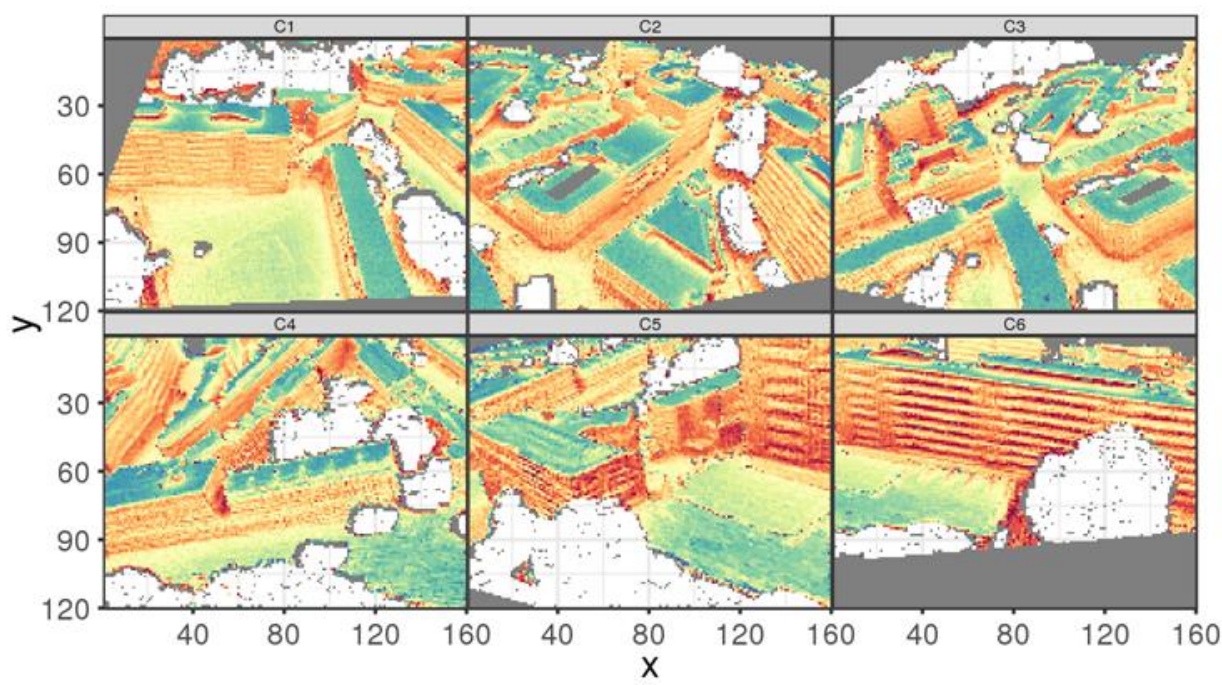

(a)
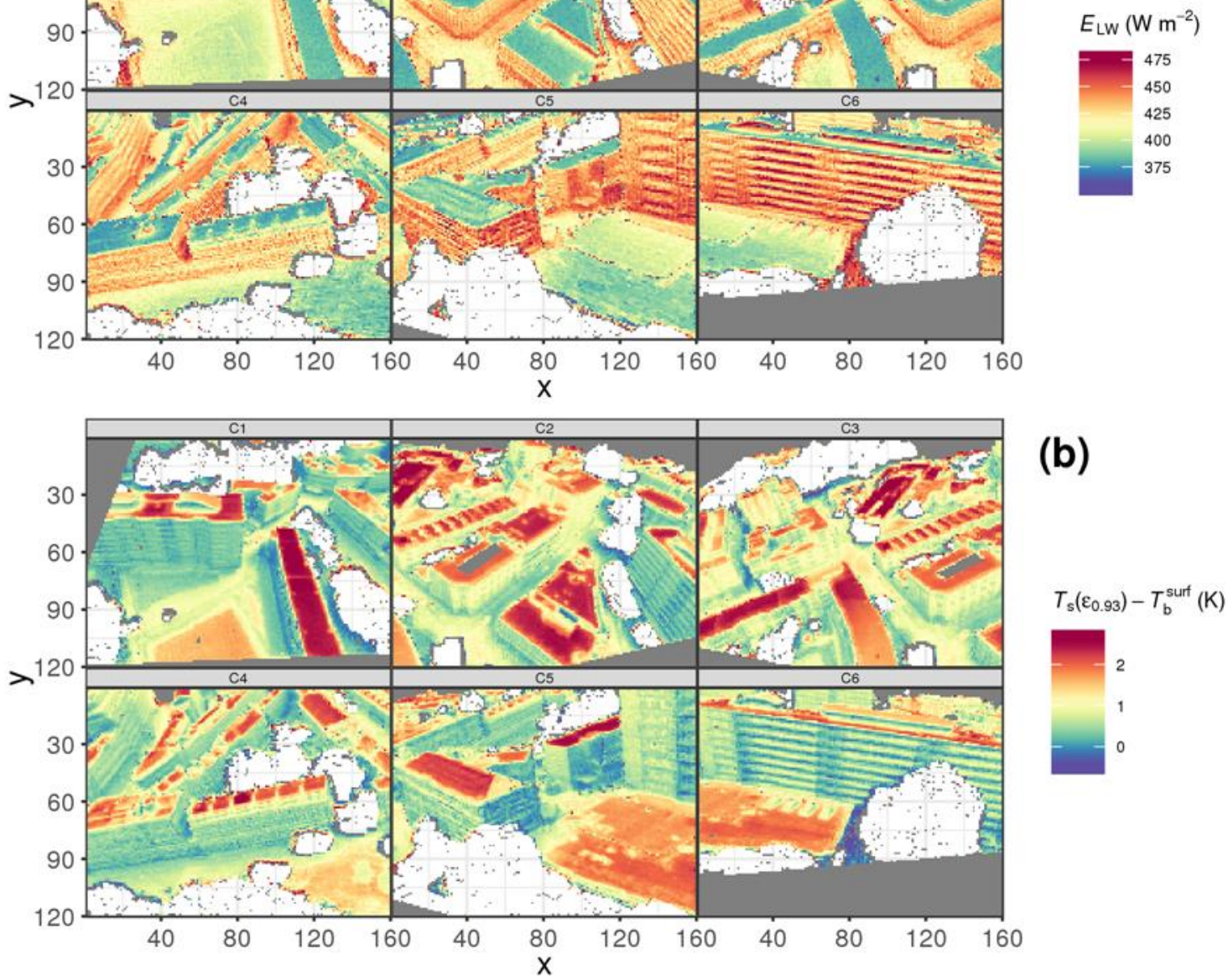

Figure 11. Observed and modelled results (27 August 2017 at 11:30) of (a) longwave irradiance ( $E_{\mathrm{LW}}$ ) from broadband hemispherical radiometer (sky component) and 3D distribution of surface brightness temperatures from the network of longwave infrared cameras (canopy component) prescribed to DART to simulate the emission, irradiance and multiple scattering processes of LWIR radiation for correction of surface brightness temperature ( $T_{\mathrm{b}}$ surf) to emissivity $(0.93)$ corrected surface temperature $\left[T_{\mathrm{s}}\left(\varepsilon_{0.93}\right)\right]$, and $(\mathrm{b}) T_{\mathrm{s}}-T_{\mathrm{b}}^{\text {surf }}$ difference.

\subsubsection{Uncertainty analysis}

The variability of $T_{\mathrm{s}}$ based on the emissivity and temperature value prescribed across the non-vegetative surfaces is evaluated for each timestep. Initially with $\varepsilon_{0.93}$, a heterogeneous distribution of surface temperature is used (Figure 12) and then repeated using the minimum ( $\left.\varepsilon_{0.89}\right)$ and maximum $\left(\varepsilon_{0.97)}\right.$ broadband emissivity values for dark impervious urban materials in the Kotthaus et al. (2014) spectral library; and repeated again (Figure 13) with an isothermal surface temperature that resolves the RT process similarly to the SVF approach of Adderley, Christen and Voogt (2015). 

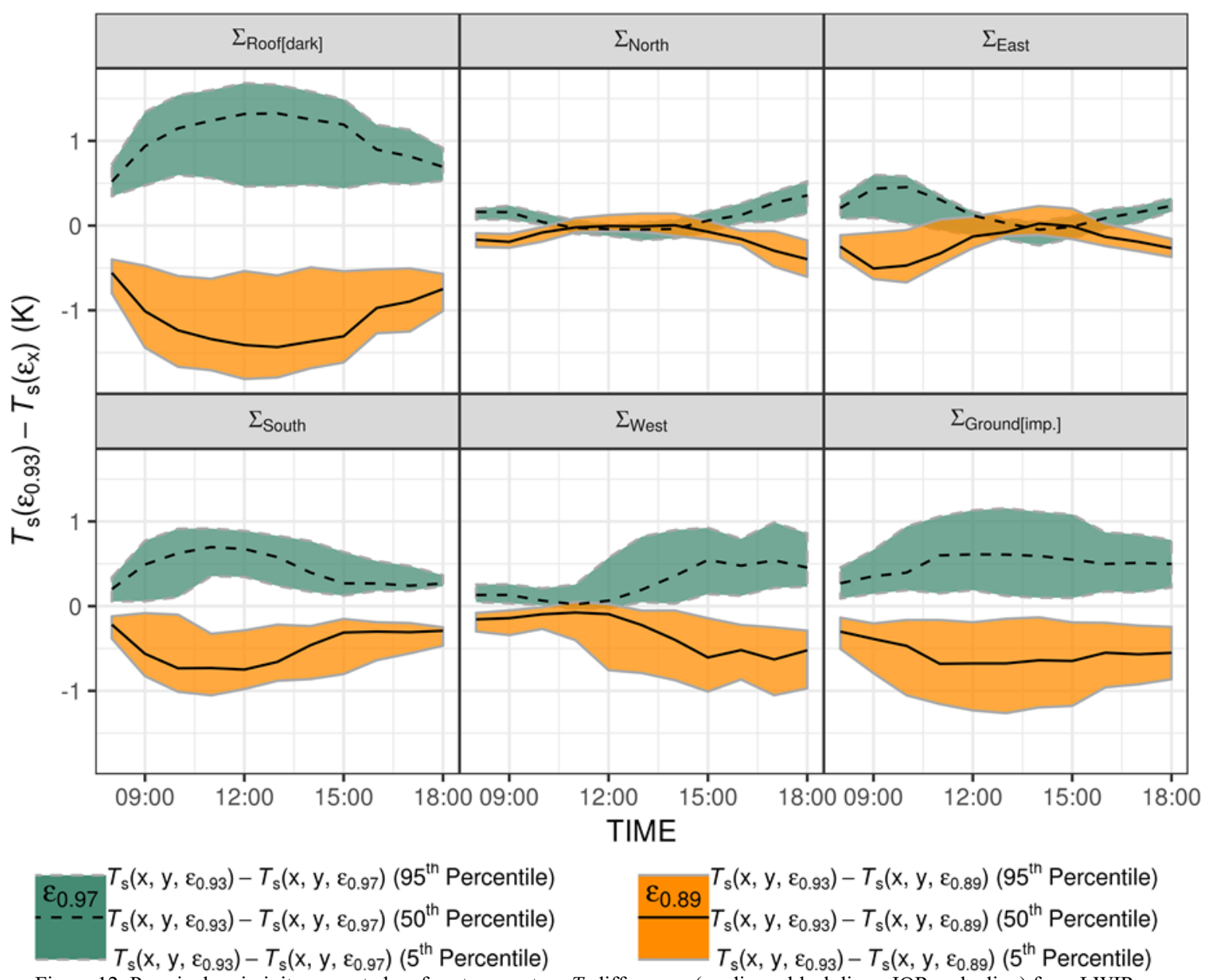

Figure 12. Per-pixel emissivity corrected surface temperature $T_{\mathrm{s}}$ differences (median = black lines, IQR $=$ shading) from LWIR camera observations (27 August 2017, $60 \mathrm{~min}$ resolution) using DART for different spectral library (Kotthaus et al., 2014) broadband emissivity values: mean ( $\varepsilon_{0.93}$ ), minimum ( $\varepsilon_{0.89}$, orange) and maximum $\left(\varepsilon_{0.97}\right.$, aqua) for non-vegetative surfaces (walls $\mathrm{E}, \mathrm{N}, \mathrm{S}, \mathrm{W}$; ground, roof).

The facet most sensitive to changes in surface emissivity is the roof as its high $T_{\mathrm{b}}{ }^{\text {surf }}$ and SVF combine with the low incoming (sky) LWIR radiation to produce large contrasts between received and emitted radiation. The emissivity effect for roof surfaces is most pronounced at 13:00 (on this day), when the median difference between the surface temperature derived using an emissivity of $0.93\left(\varepsilon_{0.93}\right)$ is $1.4 \mathrm{~K}$ higher $(1.3 \mathrm{~K}$ lower) than with $\varepsilon_{0.89}\left(\varepsilon_{0.97)}\right.$ (Figure 12). Although ground surfaces also mostly receive radiance from the cold sky, temporal variability in $T_{\mathrm{s}}(\mathrm{x}, \mathrm{y}$, $\left.\Sigma_{\text {Ground }}\right)$ is lower compared to roof surfaces as the diurnal amplitude of surface temperatures of this facet type is less because of the smaller shortwave energy receipt (Figure 10a).

For the wall facets, the magnitude of the emissivity effect is impacted by their orientation (Figure 12). Depending on the emissivity value used, the sign of the differences between surface temperatures obtained can even change throughout the day. For east- and south-facing walls the uncertainty is greatest in the morning when the surfaces are insolated and have high $T_{\mathrm{s}}$, whilst opposing walls (west and north) are shaded with low $T_{s}$. By the afternoon, differences for east-facing walls are minimised when the west-facing walls are insolated and have similar temperature to east facing walls. The asymmetry of the uncertainty for south-facing walls around solar noon, with greater uncertainty before noon, is linked to the preferential view of south-southeast walls and resulting diurnal cycle of $T_{\mathrm{b}}{ }^{\text {surf }}\left(\Sigma_{\text {South }}\right)$ (Figure 10a). Although east walls have a similar distribution of orientations to west walls, they respond differently to changes in prescribed emissivity. This is associated with the high diurnal variability of observed brightness temperatures in this class (Figure 10).

To assess the impact of variations in LWIR radiation leaving the canopy surfaces, the correction to $T_{\mathrm{s}}\left(\varepsilon_{0.93}\right)$ is performed using two different distributions of surface brightness temperature across the MW area. The "heterogeneous" temperature $\left(T_{\mathrm{b}}{ }^{3 \mathrm{D}}\right)$ is derived from the full temperature distribution $\left[T_{\mathrm{b}}\right.$ surf $\left.(\mathrm{X}, \mathrm{Y}, \mathrm{Z}, \Sigma)\right]$. This is compared to an "isothermal" case $\left(T_{\mathrm{b}}{ }^{\text {iso }}\right)$ with two classes: roof (including both $\left.\Sigma_{\text {Roof[dark] }}, \Sigma_{\text {Roofllight }]}\right)$, and "within canopy" (i.e. walls and ground). The combination of isothermal within-canopy temperatures, isotropy of surface emissivity and downwelling sky radiance means $T_{\mathrm{b}}$ iso is analogous to the SVF approach of Adderley, Christen and Voogt (2015). The isothermal distribution of temperatures eliminates strong contrasts between the walls, such as $\left\langle T_{\mathrm{b}}{ }^{\text {surf }}\left(\Sigma_{\text {south }}\right)\right\rangle$ up to $14.6 \mathrm{~K}$ greater than the median brightness temperature for the overall "within canopy" class at 11:30.

Assigning a more realistic temperature distribution $\left(T_{\mathrm{b}}{ }^{3 \mathrm{D}}\right)$ allows the heterogeneous urban canopy influences to impact the derived surface temperature $\left[T_{\mathrm{s}}\left(\mathrm{x}, \mathrm{y}, T_{\mathrm{b}}{ }^{3 \mathrm{D}}\right)\right]$ compared to the isothermal case $\left[T_{\mathrm{s}}\left(\mathrm{x}, \mathrm{y}, T_{\mathrm{b}}{ }^{\text {iso }}\right)\right]$. A reduced emissivity enhances the surface temperature differences between the heterogeneous $\left[T_{\mathrm{s}}\left(\mathrm{x}, \mathrm{y}, T_{\mathrm{b}}{ }^{\mathrm{B}}\right)\right]$ and isothermal $\left[T_{\mathrm{s}}\left(\mathrm{x}, \mathrm{y}, T_{\mathrm{b}}{ }^{\text {iso }}\right)\right]$ cases (Figure 13). As the proportion of reflected radiation increases, the effect of assigning contrasting brightness temperature distributions increases with decreasing emissivity. Simulations using 80.97 have a $5^{\text {th }}-95^{\text {th }}$ percentile range of $T_{\mathrm{s}}\left(\mathrm{x}, \mathrm{y}, \varepsilon_{0.97}, T_{\mathrm{b}}{ }^{3 \mathrm{D}}\right)-T_{\mathrm{s}}\left(\mathrm{x}, \mathrm{y}, \varepsilon_{0.97}, T_{\mathrm{b}}\right.$ iso $)$ that is typically less than $0.1 \mathrm{~K}$ (Figure 13, blue). The range for simulations using $\varepsilon_{0.89}$ $\left[5^{\text {th }}-95^{\text {th }}\right.$ percentile, $\left.T_{\mathrm{s}}\left(\mathrm{x}, \mathrm{y}, \varepsilon_{0.89}, T_{\mathrm{b}}{ }^{3 \mathrm{D}}\right)-T_{\mathrm{s}}\left(\mathrm{x}, \mathrm{y}, \varepsilon_{0.89}, T_{\mathrm{b}}{ }^{\text {iso }}\right)\right]$ is greatest for $\Sigma_{\text {Ground[imp.] }}$ surfaces (up to $0.4 \mathrm{~K}$ at $\left.11: 00\right)$. As $\Sigma_{\text {Roof }}$ have low wall view factors, the sensitivity of this class to incoming LWIR radiation from within canopy surfaces is low throughout the day. The emissivity effect for the other within-canopy surfaces varies through the day with the brightness temperature of the opposite facets (Figure 10a). Given the surface emissivity impact increases if the facing wall has a very different temperature (Figure 12), the relative temperature distribution between walls is important. For $\Sigma_{\text {North }}$, the $5^{\text {th }}$ percentile of $T_{\mathrm{s}}\left(\mathrm{x}, \mathrm{y}, \varepsilon_{0.89} T_{\mathrm{b}}{ }^{\text {iso }}\right)$ overestimates the $5^{\text {th }}$ percentile of $T_{\mathrm{s}}\left(\mathrm{x}, \mathrm{y}, \varepsilon_{0.89}, T_{\mathrm{b}}{ }^{3 \mathrm{D}}\right)$ by $0.25 \mathrm{~K}$ in the period 11:30 - 12:00. This effect of temperature distribution within the canopy on the emissivity correction can therefore be larger than when changing the actual emissivity value used for north walls, as the $5^{\text {th }}$ and $95^{\text {th }}$ percentile differences in $T_{\mathrm{s}}\left(\mathrm{x}, \mathrm{y}, \varepsilon_{0.89}\right)-T_{\mathrm{s}}\left(\mathrm{x}, \mathrm{y}, \varepsilon_{0.97}\right)$ are within $\pm 0.2 \mathrm{~K}$ during the same 11:30 - 12:00 period (within $\pm 0.5 \mathrm{~K}$ for 08:00 - 18:00) (Figure 12). These results highlight that assuming emitted radiation is only a 
function of SVF does not account for the full complex thermal heterogeneity of the urban canopy and can contribute towards uncertainty in the emissivity correction.

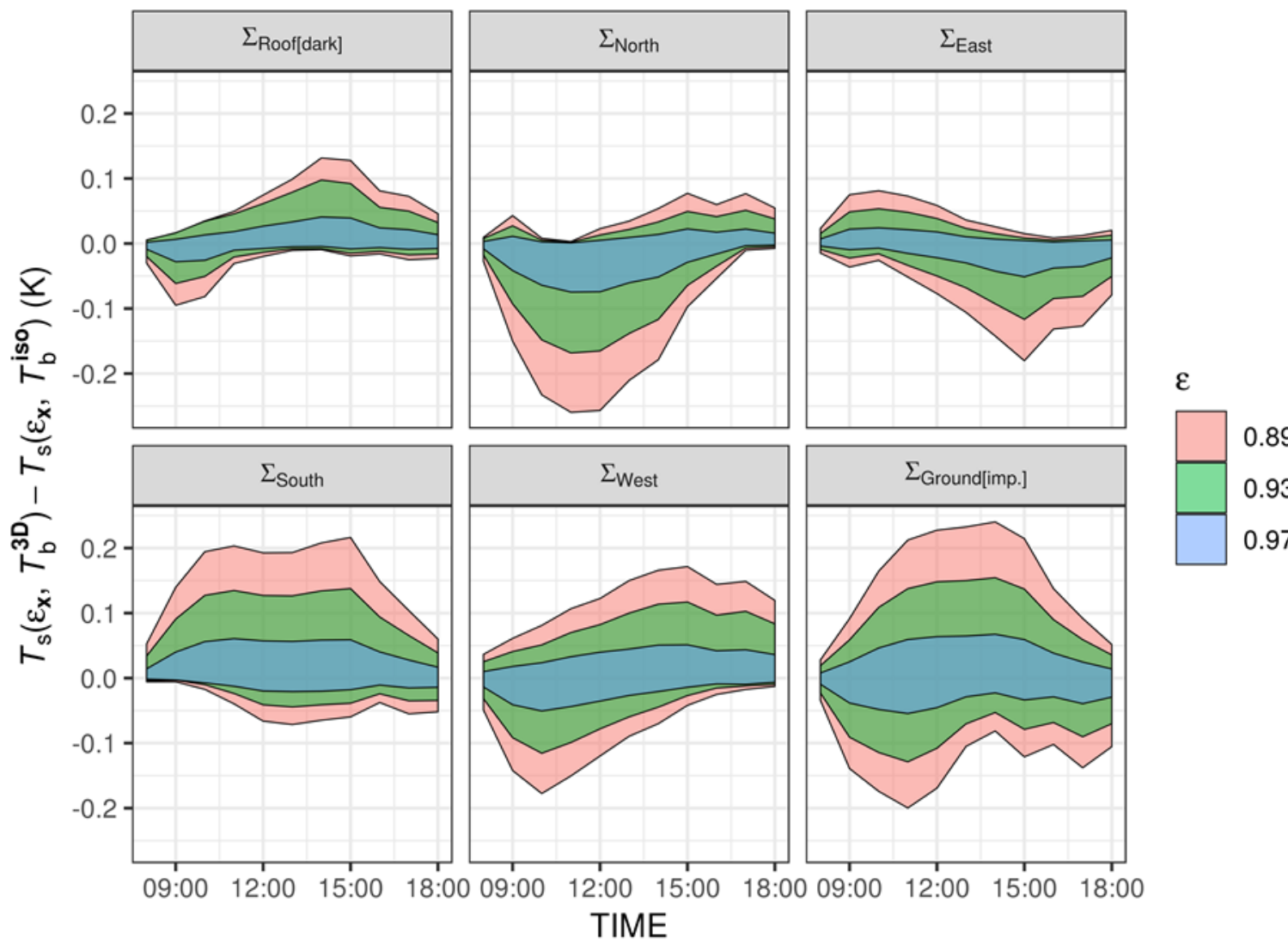

Figure 13. Difference in surface temperature $T_{\mathrm{s}}(60 \mathrm{~min}$ resolution, 27 August 2017) derived from LWIR camera observations based on emissivity $(\varepsilon)$ corrections with a heterogeneous $\left(T_{\mathrm{b}}{ }^{3 \mathrm{D}}\right)$ and isothermal $\left(T_{\mathrm{b}}\right.$ iso $)$ assumption, using a range of $\varepsilon$ values (non-vegetative surfaces) from the dark impervious materials in the Kotthaus et al. (2014) spectral library. As observations are not areally weighted, the lower and upper extent of the distributions can still represent large fractions of the surface seen by the LWIR cameras.

\section{Discussion}

\subsection{Flexibility in the correction framework}

The correction methodology can be used for a wide range of observation sites and applications beyond the case-study.

For the atmospheric correction, climate model or reanalysis data could be used as input data instead of the more costly and challenging vertical extrapolation of in-situ weather station observations applied here. A trade-off may exist with model grid resolution and skill, but the horizontal and vertical distribution of air temperature, pressure and water vapour from the model may be more accurately resolved than by some observations. Alternatively, standard gas and aerosol models are available within the DART database. In addition to vertical atmospheric variations, the 3D RT approach is unique in that any horizontal variability (e.g. associated with localised point sources or distributions of water vapour or soot) can be specified. We aimed for atmospheric correction processing at close to real time $(<5$ min simulation time $)$. The final $\sim 12$ min simulation time per timestep across all cameras means the sensitivity of the model resolution (e.g. voxels, number of rays) to the simulated results requires more investigation and there is much scope for reduction in simulation time.

The emissivity correction uncertainty analysis could be expanded to consider more spatially variable materials and anisotropy in surface emissivity such as specular reflections from glass. The discrete ordinate (DO) nature of DART means spectral properties and scattering phase functions can be determined either manually or from the DART database. In general, the distribution of material properties for the correction is only limited by the input data. As more detailed optical property information become available, this could be incorporated into other datasets and classification techniques. Given a primary benefit of ground-based RS is that observations can be made of the full 3D structure including vertical surfaces, a description of the material composition of the vertical surfaces is particularly important and can be used here. Google Street View has been applied in urban climate studies (Gong et al., 2018; Zeng et al., 2018) and could be used to obtain structure and material composition information for within-canopy surfaces across large areas (Lindberg et al., 2019).

To further understand uncertainties with emissivity correction from changes in surface temperature distributions, the surface temperature distribution can be pre-processed by DART (e.g. Wang, Chen and Zhan, 2018) or by user-defined approaches (Morrison et al., 2018) to resolve its variability from shadow patterns.

We assumed downwelling radiance from the sky to be an isotropic source to isolate variance from the other effects studied. However, an anisotropic sky radiance can be prescribed which uses the full above-BOA (bottom of atmosphere) radiative transfer capabilities of DART as opposed to the broadband sky irradiances used here.

Additional sensitivity analyses considering all of these processes could contribute to a benchmarking effort to reduce simulation times (each emissivity correction takes $\sim 12 \mathrm{~h}$ using 4 cores, $40 \mathrm{~GB}$ ram) and allow for larger domain areas. This could involve simplifications (e.g. using first-order scattering of LWIR radiation only) and/or reduction of model resolution (e.g. fewer voxels and lower density of rays).

\subsection{Future application of corrected ground-based observations}

To enhance the applicability of ground-based thermography observations for studying physical exchange processes, the correction of atmospheric and emissivity effects is crucial. Surface kinetic temperature $\left(T_{\mathrm{s}}\right)$ from high resolution LWIR RS on ground-based platforms in 
urban areas is increasingly required for a wide range of applications. The role of complex geometry to 3D RT processes is important to understand, particularly as modelling typically uses low LOD geometry restricted by data availability (e.g. Ghandehari, Emig and Aghamohamadnia, 2018) or the nature of the model as a 2D (Harman, Best and Belcher, 2004) or simplified 3D (Krayenhoff and Voogt, 2007; Aoyagi and Takahashi, 2012) schemes. The corrected ground-based observations are expected to have application in the following specific fields: ground-truthing of airborne or space-borne surface temperatures by assessment of the anisotropy (directional variability) of upwelling LWIR radiation (Lagouarde et al., 2014; Krayenhoff and Voogt, 2016), estimation of complete urban surface temperatures (Voogt and Oke, 1997), evaluation of urban radiation schemes (Hogan, 2019) and urban surface (e.g. Masson, 2000; Harshan et al., 2018) and building (Bueno et al., 2012) energy balance models, studies of shadow hysteresis effects (Meier, Scherer and Richters, 2010), inputs to unstably stratified large eddy simulations (Gronemeier, Raasch and Ng, 2017) and data assimilation (e.g. Ghent et al., 2010; Li and Bou-Zeid, 2014).

\section{Conclusions}

As high-resolution thermal imagery starts to be gathered in urban settings for long periods, detailed automatic correction techniques are required. Buildings and vegetation often have different patterns and heterogeneity across a city (cf. city centre to residential), meaning flexible methods are needed to account for the specific urban morphology. Here, a radiative transfer model is used to convert brightness temperature observations to kinetic surface temperature from a network of seven thermal cameras deployed in a complex urban area. The methods applied to correct for the emission and absorption of radiation between the surface and camera (atmospheric correction) and longwave scattering from non-black-body surfaces ("emissivity" correction) are advantageous over earlier work as: (a) the correction is applied within one modelling framework; (b) a large number of cameras with varying properties can be corrected simultaneously with minimal increase to computational cost; (c) a very high level of detail and realistic surface model is created and explicitly represents buildings with sloped roofs, micro-scale structures (e.g. balconies), and vegetation; (d) multiple scattering of radiation within the urban atmosphere and between building structures is accounted for; and (e) heterogeneous temperature distributions within the urban canopy are used.

The conclusions drawn from this work are:

- The atmospheric correction can be large (e.g. $2.97 \mathrm{~K}$ ) over relatively short path lengths (e.g. $155 \mathrm{~m})$. The proposed correction is demonstrated to have good agreement with simultaneous observations at a very short path length (mean absolute error $0.39 \mathrm{~K}$ ). A seasonally varying evaluation is needed.

- A high level of detail surface geometry model allows for accurate pixel path length, even with complex features (e.g. sloped roofs), giving much more accurate atmospheric corrections compared to more simplified geometry (e.g. assuming flat roofs may make path lengths much longer and for this study could give errors over $3 \mathrm{~K}$ ).

- The emissivity correction has a diurnal pattern and varies by surface type. For example, on a clear-sky day the correction is greatest around midday, with roofs over $3 \mathrm{~K}$ warmer when corrected.

- Roofs likely have the greatest uncertainty in estimated $T_{\mathrm{s}}$. Because of their high sky view factor, error sources are the prescribed material emissivity and longwave sky irradiance. A more general error source is rapid changes in camera body temperature during clear-sky mornings which correspond to relatively poor agreement between modelled and observed atmospheric correction magnitudes $(\sim 1 \mathrm{~K}$ difference). More work on the calibration and housing of longwave infrared cameras for outdoor settings is required.

- A baseline correction using an emissivity of $0.93\left(\varepsilon_{0.93}\right)$ across all built surfaces has an order of $\pm 1.4 \mathrm{~K}$ variation compared to a correction using expected emissivity values for dark building materials ( $\varepsilon_{0.89}$ to $\varepsilon_{0.97}$, Kotthaus et al., 2014).

- Driven by varied surface temperatures and sky view factors, the irradiance across the surfaces is highly variable (intra-pixel differences $>$ $70 \mathrm{~W} \mathrm{~m}^{-2}$ ). South facing walls are up to $17 \mathrm{~K}$ warmer than north walls. The latter generally follow the air temperature and have cooler temperature when corrected $(<-0.25 \mathrm{~K}$ around midday). By removing the surface temperature variation, which is analogous to a sky view factor correction (Adderley, Christen and Voogt, 2015), the correction changes by around $0.25 \mathrm{~K}(0.1 \mathrm{~K})$ using the low (high) emissivity values of $\varepsilon 0.89(\varepsilon 0.97)$. Thus, the uncertainty introduced when using a simplified SVF approach could be larger than the uncertainty introduced from material emissivity choice.

Overall, the technique introduced is flexible and corrects for atmospheric and emissivity effects at an unprecedented level of detail. Once a "model world" is defined that describes the observational area (i.e. observed surface geometry and sensor perspective), the use of an anisotropic radiative transfer model (DART, Gastellu-Etchegorry et al., 2015) simplifies this challenging and complex correction procedure within a single model interface. There is flexibility in the choice of model inputs and parameters including the: 3D distributions of atmospheric optical properties, surface temperatures and (directional) surface emissivity; anisotropic downwelling longwave radiance from the sky; surface and vegetation geometry; remote sensing observations from different platforms and design (e.g. cameras and narrow-wide FOV radiometers), which may be important for configurations where the observations and model resolve more detailed material properties, including anisotropic emissivity and specular reflections. By using a network of cameras with observations of a broad range of urban surface types and surface-camera distances, the approach encompasses a range of these parameters which will invariably be relevant as a benchmark for future applications.

\section{List of symbols and acronyms [units]}

$\begin{array}{ll}\text { 3D } & \text { Three dimensional } \\ \text { AGL } & \text { Above ground level }(\mathrm{m}) \\ B_{\lambda} & \text { Black-body Planck radiance }\left[\mathrm{W} \mathrm{m}^{-2} \mathrm{sr}^{-1} \mu \mathrm{m}^{-1}\right] \\ \text { BB } & \text { Black-body } \\ \text { BCT } & \text { Observation site for weather data }(\sim 1 \mathrm{~km} \text { outside study area) } \\ \text { BOA } & \text { Bottom of atmosphere } \\ \text { C\# } & \text { Nonspecific camera }(\mathrm{C}) \text { and unique reference number (\#) } \\ \mathrm{c}_{1} & \text { First radiation constant }\left[1.191042 \times 10^{9} \mathrm{~W} \mathrm{~m}^{-2} \mathrm{sr}^{-1} \mu \mathrm{m}^{-1}\right] \\ \mathrm{c}_{2} & \text { Second radiation constant }\left[1.4387770 \times 10^{4} \mu \mathrm{m} \mathrm{K}^{-1}\right. \\ \text { CUB } & \text { Observation site within the study area } \\ \text { DART } & \text { Discrete Anisotropic Radiative Transfer model } \\ \text { DO } & \text { Discrete ordinate } \\ \text { DSM } & \text { Digital surface model (3D vector-based) } \\ \Delta T_{\mathrm{b}} \text { cam } & \text { Atmosphere effect for C2 (no correction) as } T_{\mathrm{b}}{ }^{\text {cam }}(\mathrm{C} 2)-T_{\mathrm{b}} \text { surf }(\mathrm{C} 7)[\mathrm{K}] \\ \Delta T_{\mathrm{b}}{ }^{\text {surf }} & \text { Atmosphere effect corrected for C2 as } T_{\mathrm{b}}{ }^{\text {surf }}(\mathrm{C} 2)-T_{\mathrm{b}}{ }^{\text {surf }}(\mathrm{C} 7)[\mathrm{K}] \\ E_{\mathrm{LW}} & \text { Broadband incoming longwave radiation flux (irradiance }\left[\mathrm{W} \mathrm{m}^{-2}\right] \\ E_{\mathrm{LW}}{ }^{\text {sky }} & \text { Broadband incoming longwave radiation flux (irradiance) from sky }\left[\mathrm{W} \mathrm{m} \mathrm{m}^{-2}\right] \\ \varepsilon & \text { Emissivity }\end{array}$




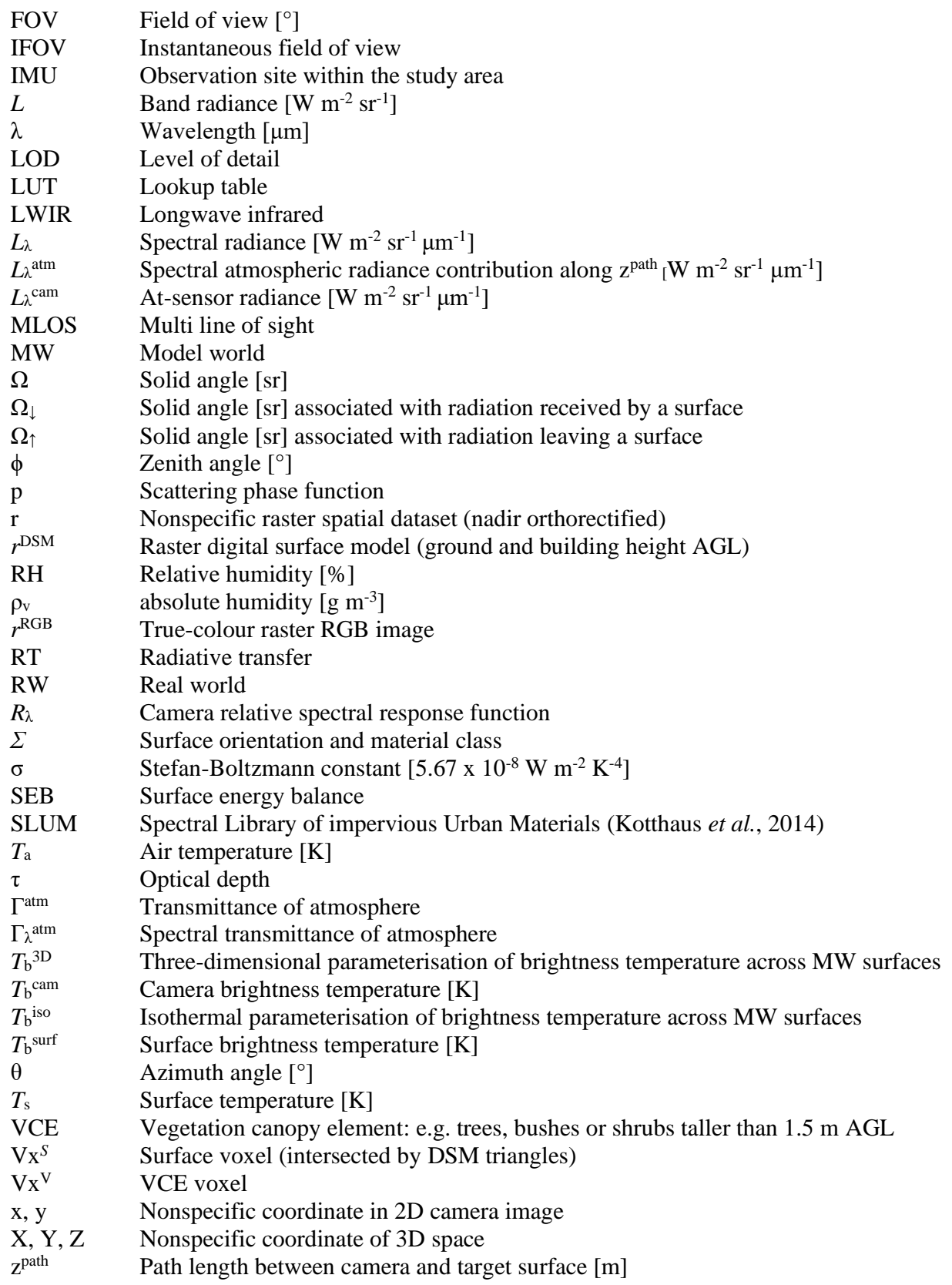

\section{Acknowledgements}

This work is funded by Newton Fund/Met Office Climate Science for Service Partnership China (CSSP China, SG), EPSRC DARE, EU H2020 UrbanFluxes, EUf7 emBRACE, NERC ClearfLo, NERC/Belmont TRUC, and the National Research Foundation (NRF) Singapore through the Singapore-MIT Alliance for Research and Technology's Centre for Environmental Sensing and Modeling (SMART-CENSAM) interdisciplinary research program. We thank Islington Council (Esther Lamontagne and Paul Stokes) and City University (Prof. Sir Paul Curran and Dean Beeden) for arranging access to observation sites, Daniel Clewley of the NERC Airborne Research Facility for assistance with the airborne hyperspectral dataset, Kjell Zum Berge (University of Reading) for assistance with the observations and Bryam Orihuela for assistance with the 3D modelling. We thank NERC ARSF, Martin Wooster and all those who contributed to the airborne measurement campaign 2010/154. Further supplementary data available on Zenodo (Morrison, 2019).

\section{Appendices}

\section{Appendix A. Camera image emissivity maps from the DART model}

An emissivity map is required for the emissivity correction of longwave infrared (LWIR) cameras. It needs to have the camera image perspective with emissivity values for each pixel informed by the surfaces observed and their associated material and optical properties. With an isotropic emissivity, the view angle of the camera does not alter the observed surface emissivity, thus an emissivity map can be created that is referenced to any prior image classification (e.g. surface orientation and material maps, Figure 4).

For anisotropic emissivity situations, DART is used to pre-calculate an emissivity map considering the view angle dependence of surface emissivity for any given camera. For this, the DART model world (MW) surfaces are configured to have materials with scattering phase functions $(p)$ to give an anisotropic and spectral emissivity $\varepsilon \lambda, p$. A DART simulation similar to that used for the non-black-body (non-BB) radiance calculation $\left(L_{\lambda}{ }^{\mathrm{DART}}(\mathrm{x}\right.$, $\left.\mathrm{y}, \Omega_{\uparrow}, \varepsilon_{\lambda, p}<1\right), \mathrm{W} \mathrm{m}^{-2} \mathrm{sr}^{-1} \mu \mathrm{m}^{-1}$, Section 3.3) is used and adjusted to have: one known surface temperature $\left(\bar{T}_{s}\right)$ across all surfaces, no sky emission (sky brightness temperature $T_{\mathrm{b}}$ sky $=0 \mathrm{~K}$ ) and no scattering events. This gives a non-BB surface-leaving radiance product $\left[L_{\lambda}{ }^{\mathrm{DART}}\left(\mathrm{x}, \mathrm{y}, \Omega_{\uparrow}, \varepsilon_{\lambda, p}<1\right.\right.$, $\left.\left.T_{\mathrm{b}}{ }_{\mathrm{b}}^{\text {sky }}=0, \overline{T_{S}}\right)\right]$ used to determine $\varepsilon_{\lambda, p}(\mathrm{x}, \mathrm{y})$ across the MW camera perspective with:

$$
\varepsilon_{\lambda, p}(\mathrm{x}, \mathrm{y})=\frac{L_{\lambda}^{\mathrm{DART}}\left(\mathrm{x}, \mathrm{y}, \Omega_{\uparrow}, \varepsilon_{\lambda, p}<1, T_{\mathrm{b}}^{\mathrm{sky}}=0, \bar{T}_{s}\right)}{B_{\lambda}\left(\bar{T}_{s}\right)}
$$


where $B_{\lambda}\left(\bar{T}_{S}\right)$ is the expected at-sensor Planck radiance for a black-body (i.e. $\varepsilon \lambda, \mathrm{p}=1$ ) at the given homogeneous temperature. All other terms are defined in notation list.

\section{Appendix B. Creation and classification of complex 3D surface and vegetation models}

Photogrammetry techniques are used to build a high level of detail (LOD) description of the surface geometry of the central London study area.

\section{B.1. Creation}

Urban surface geometry of buildings, roads, and vegetation (Figure B-1) are resolved to include sub-facet details (e.g. sloped roofs and balconies). The model world (MW) area has an initial horizontal extent of $450 \times 450 \mathrm{~m}$.
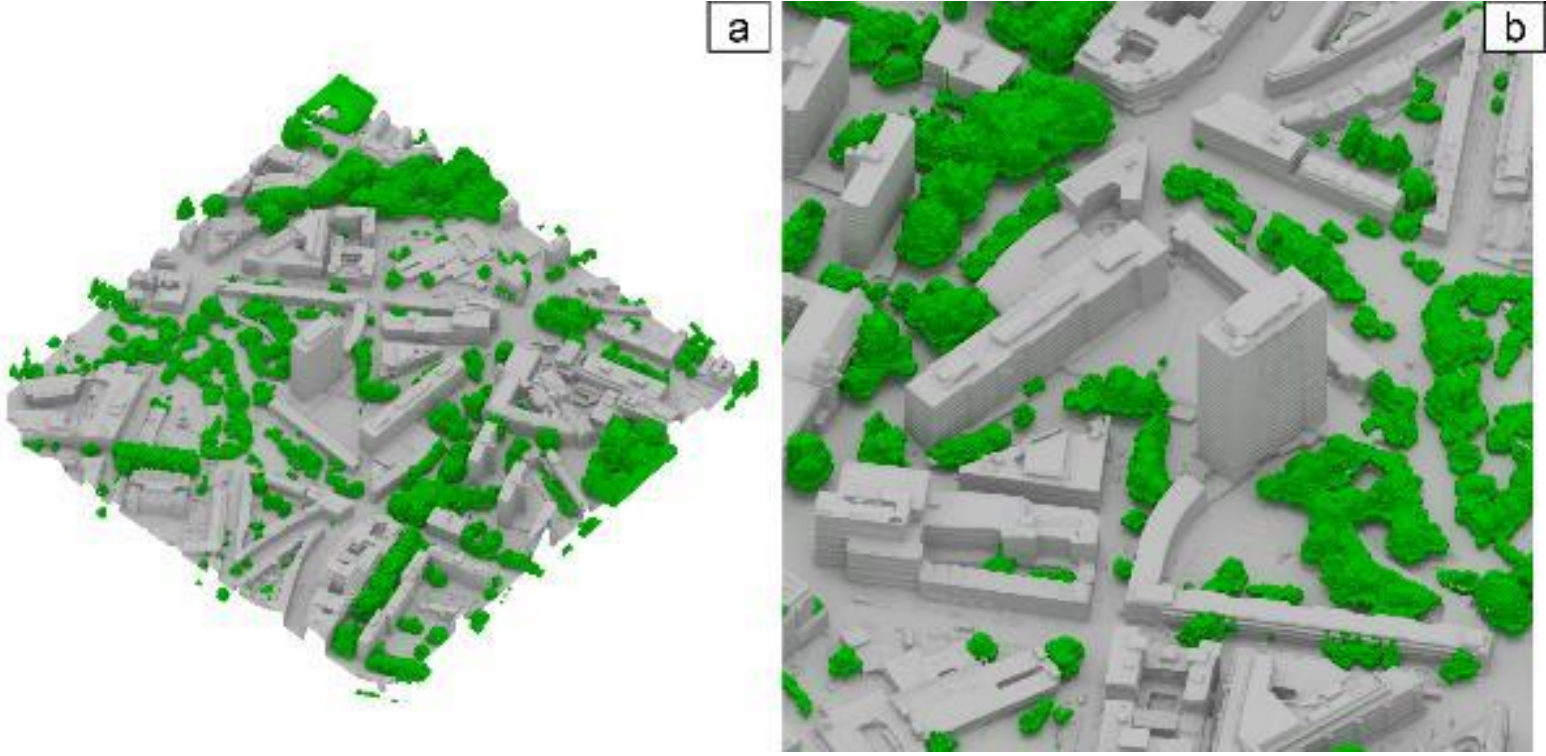

Figure B-1. Urban surface geometry includes a vector-based 3D surface model (grey) and voxel-based model of vegetation canopy elements (green). The central London (UK) study area rendered using Blender (Blender, 2018) showing: (a) full extent $(450 \mathrm{~m} \mathrm{x} 450 \mathrm{~m}$ ) and (b) more detail around the centre of the area.

Initially, the MW area is split into nine $150 \mathrm{~m}$ x $150 \mathrm{~m}$ georeferenced tiles ( 3 x 3 array). In Google Earth Pro (Google, 2019a), a programmed "tour" takes 32 images per tile at $200 \mathrm{~m}$ from the centre of each tile with a $45^{\circ}$ zenith angle at $11.25^{\circ}$ azimuth angle steps (tile height: mean height above ground level (centre), derived from Google Maps API, Google, 2019b). The tour, saved in .kml file format, can be read by Google Earth. By taking images around a centre point, most of the Google Earth 3D surface present within a tile is captured (Figure B-2).

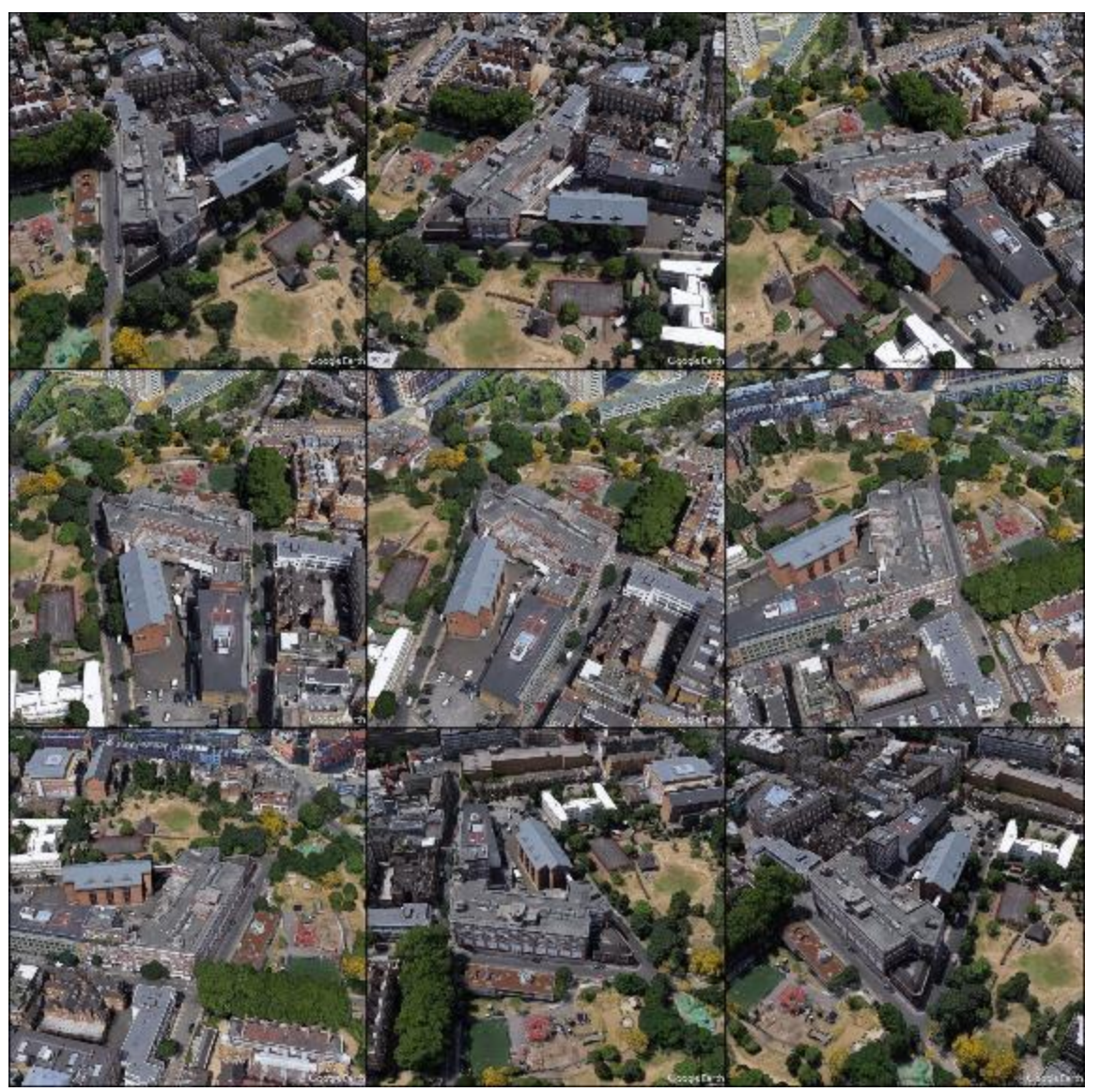

Figure B-2. Nine sample images captured by Google Earth using a pre-programmed Google Earth "tour". Images are used to create a dense point cloud "tile" in Agisoft PhotoScan Professional (Figure B-3). 
Agisoft PhotoScan Professional (Agisoft LLC, 2013) photogrammetry software suite ("PhotoScan") Version 1.3 .4 build 506 is used to process the Google Earth images. The relative position and rotation of the cameras stored in the. $\mathrm{kml}$ file is verified by Photoscan using unsupervised detection of inter- and intra-image control points. For each tile, image depth mapping with Photoscan is used to construct a dense 3D point cloud (120,000 - 160,000 points) of Google Earth surfaces containing the 3D coordinate (X, Y, Z), surface normal and RGB attributes (Figure B-3).

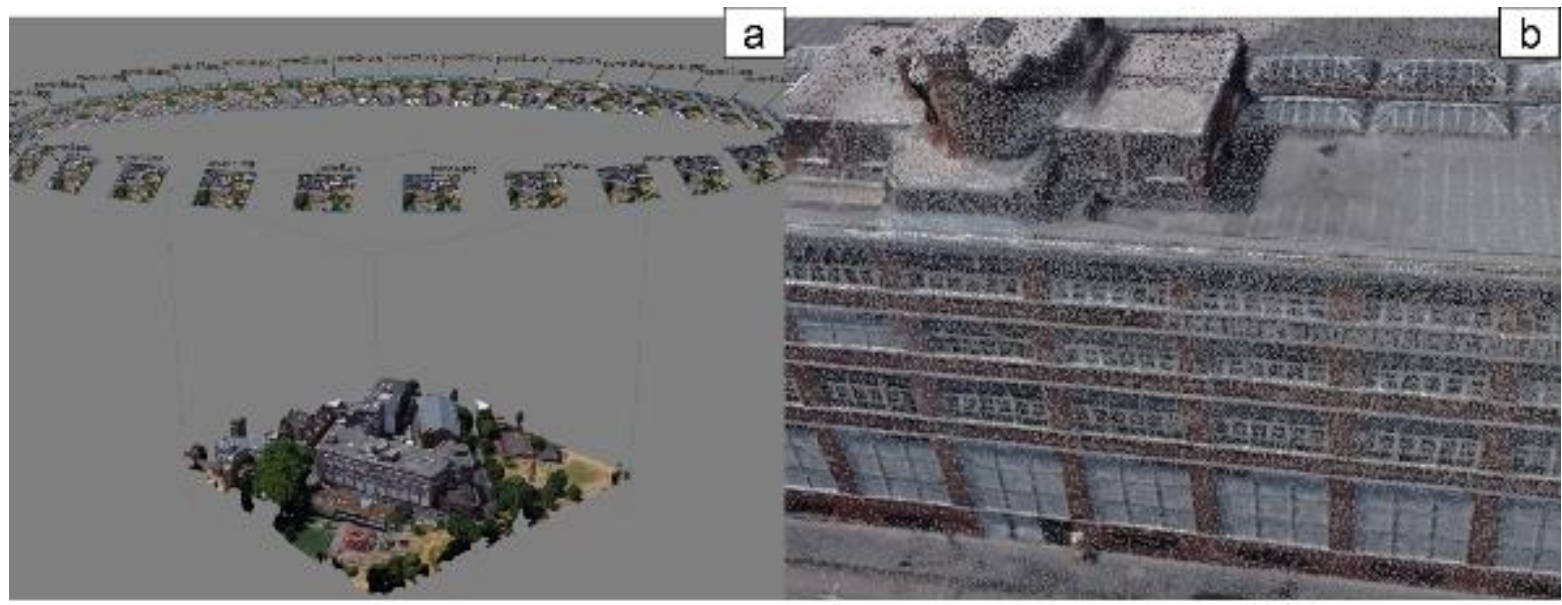

Figure B-3. Screen captures from the Agisoft PhotoScan Pro user interface (Version 1.3.4 build 506) with (a) a dense point cloud (1.56 x $10^{7}$ points) across a 150 x $150 \mathrm{~m}$ "tile" centred on WGS UTM $31 \mathrm{~N}$ coordinates $(\mathrm{x}=284450, \mathrm{y}=5712800)$ with the camera position for the Google Earth images shown above, and (b) a close-up of the sample dense point cloud.

Point clouds for each tile are rasterised at $0.2 \mathrm{~m}$ resolution to give raster $(r)$ digital surface models $\left(r^{\mathrm{DSM}}\right.$, e.g. Figure B-4a) for each tile, which are then merged.

As the Google Earth images only sample the top and sides of convoluted urban surfaces, the complete vegetation canopy is underrepresented by the point cloud. Thus, vegetation canopy points are extracted from the point cloud and modelled separately. Ground points within each point cloud are automatically classified with Photoscan and rasterized to create a raster digital elevation model $\left(r^{\mathrm{DEM}}\right)$ at $0.2 \mathrm{~m}$ resolution $\left(r^{\mathrm{DEM}}\right.$, Figure B-4b) which is aggregated to $5 \mathrm{~m}$ using the median values and resampled back to $0.2 \mathrm{~m}$. Green vegetation canopies are selected using an RGB colour filter applied to an orthorectified true-colour raster of the Google Earth surface ( $r^{\mathrm{RGB}}$, Figure B-4d) combined with an above ground level (AGL) height threshold $\left(\left[r^{\mathrm{DSM}}-r^{\mathrm{DEM}}\right]>1.5 \mathrm{~m}\right)$. Manual digitisation is used to select any shaded or non-green vegetation not selected, and to deselect any raster cells incorrectly identified (e.g. artificial turf). The combined automated filter and manual digitised vegetation forms a vegetation map $(r$ VEG $)$. Any point cloud points with horizontal coordinates that intersect a vegetation cell from $r^{\mathrm{VEG}}$ are moved to the height of the corresponding $r^{\mathrm{DEM}}$ cell, to produce a modified point cloud that excludes all vegetation canopy elements (VCE). A VCE is defined as any vegetation taller than $1.5 \mathrm{~m}$ AGL (e.g. trees, bushes or shrubs).

The modified point cloud is converted to a 3D vector DSM using Poisson surface reconstruction (Kazhdan and Hoppe, 2013). As surfaces near $(<10 \mathrm{~m})$ each edge of the DSM extent are poorly reconstructed with this method, they are removed; hence, the final horizontal extent of the DSM is $430 \times 430 \mathrm{~m}$.

The geometry of $\mathrm{VCE}$ is contained with a $3 \mathrm{D}$ array of voxels $\left(\mathrm{Vx}^{\mathrm{V}}\right)$ at $\Delta \mathrm{X}=\Delta \mathrm{Y}=1 \mathrm{~m}, \Delta \mathrm{Z}=0.1 \mathrm{~m}$, that are either filled with $\mathrm{VCE}$ or empty. $r^{\mathrm{VEG}}$ cells that intersect $\mathrm{Vx}^{\mathrm{V}}$ give the horizontal distribution of $\mathrm{VCE}$. The vertical distribution of $\mathrm{Vx}^{\mathrm{V}}$ uses a canopy top and base height, between which all voxels are filled. The canopy top is assumed to be the $95^{\text {th }}$ percentile height of the corresponding $r^{\mathrm{DSM}}$ and $r^{\mathrm{VEG}}$ cells. The canopy base height is determined for each VCE. Initially, individual VCE crowns are identified by applying a local maxima filter (Roussel and Auty, 2018) to $r^{\mathrm{DSM}}$ cells mapped as $r^{\mathrm{VEG}}$. The horizontal extent of each VCE crown, determined using a watershed algorithm (Plowright, 2018), produces a polygon outline for each (Figure B-4d). The "Virtual London" building footprint model (Evans, Hudson-Smith and Batty, 2006; Figure 2a) is used to determine if a VCE is on top of or near a building. A VCE polygon that intersects $>90 \%$ of the area of a building footprint polygon is assumed to originate from a roof surface. The base height of each $\mathrm{Vx}^{\mathrm{V}}$ within a roof VCE is set to the $25^{\text {th }}$ percentile height of all $r$ DSM pixels within $0.5 \mathrm{~m}$ of the roof VCE perimeter. For the voxels within each non-roof VCE, the base or "trunk" height is assumed as 0.25 of the $95^{\text {th }}$ percentile height of $r^{\mathrm{DSM}}$ pixels within the VCE polygon. 

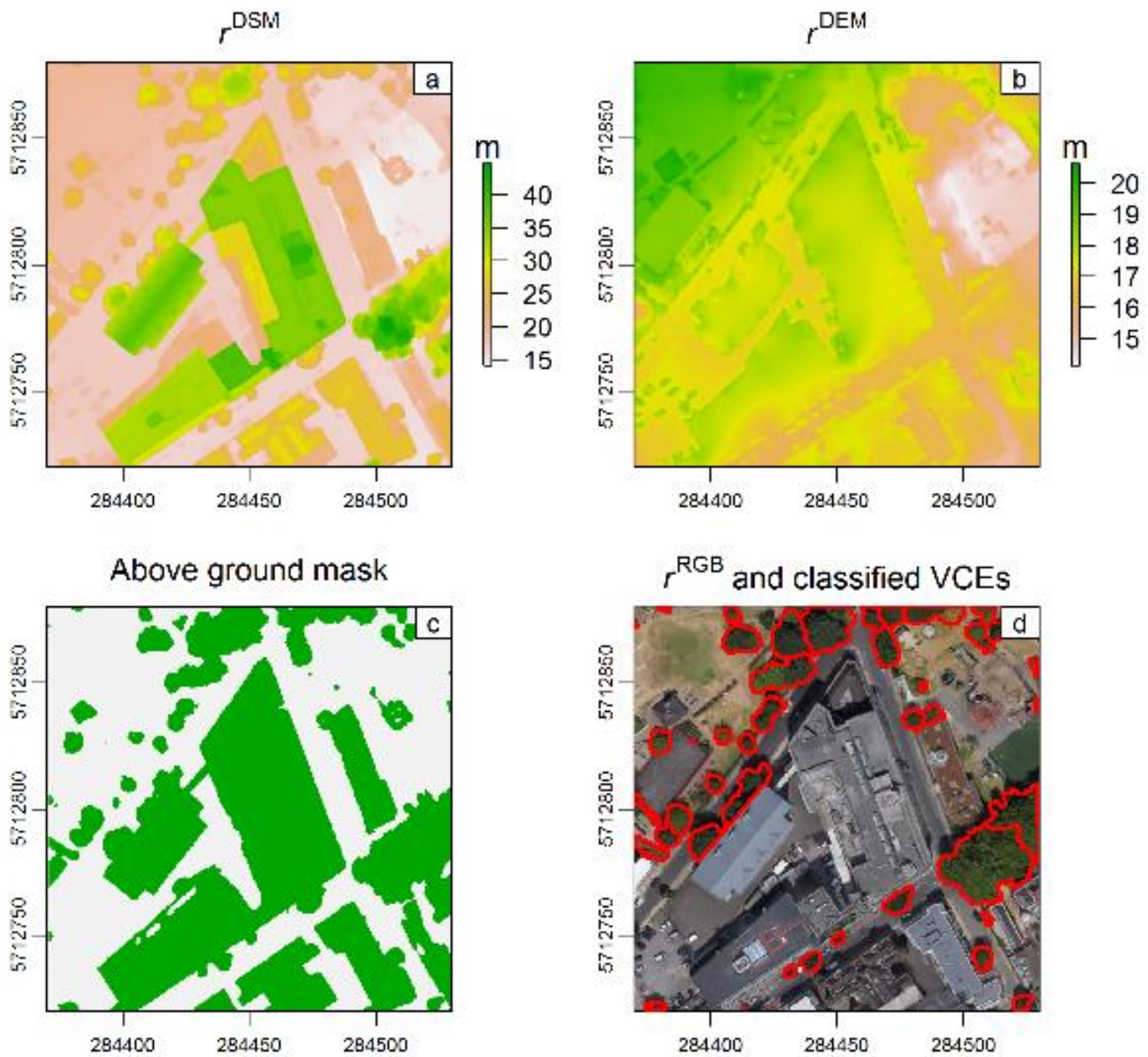

Figure B-4. Raster ( $r$ ) products for one $150 \times 150 \mathrm{~m}$ "tile" of the larger study area, photogrammetrically processed from the 3D point cloud with (a) digital surface model $\left(r^{\mathrm{DSM}}\right)$, (b) base resolution $(0.2 \mathrm{~m})$ digital elevation model $\left(r^{\mathrm{DEM}}\right)$, (c) above ground (green) surfaces, and (d) orthoimage determined by Agisoft using the raw Google Earth images and classified vegetation canopy elements (VCE, red lines). All rasters are (i) used to determine a 3D distribution of VCE (ii) shown in grid coordinate system UTM $31 \mathrm{~N}(\mathrm{~m})$ and (iii) above sea level (m) where appropriate.

\section{B.2. Classification}

The classified London surface geometry (Figure 2c) is created, after each DSM triangle is assigned an "orientation" (either north, east, south or west facing wall, roof or ground) using Blender 3D modelling software version 2.79 (Blender Foundation, 2018, hereafter "Blender"). The orientation of each triangle is defined by the smallest angular difference between the normal of a DSM triangle and the normal of each cardinal and upward (downward) direction. To differentiate upward-facing triangles as roof or ground, the height of the centroid of each upward facing triangle (i.e. triangles not assigned a cardinal orientation) is compared to the height of the corresponding $r^{\mathrm{DSM}}$ (e.g. Figure B-4) cell. A triangle is classified as ground if its centroid height is within $2 \mathrm{~m}$ of the corresponding $r^{\mathrm{DSM}}$ cell. Remaining upward triangles are assigned as roofs.

Land cover $\left(r^{\mathrm{LC}}\right)$ and hyperspectral reflectance $\left(r_{\lambda}{ }^{\omega}\right)$ maps are used to assign simple materials characteristics of: Roof [light $\mid$ dark] and Ground [imp. (impervious) $\mid$ grass]. $r^{\mathrm{LC}}$ contains built surfaces, grass and "tree" classes at $4 \mathrm{~m}$ resolution (Lindberg and Grimmond, 2011). As VCE are modelled (Section B.1) and classified separately, the ground below each VCE within $r^{\mathrm{LC}}$ is assigned as Ground[grass] for pixels that intersect the Ordnance Survey Greenspace dataset (Ordnance Survey, 2018) or otherwise as impervious (Ground[imp.]). $r_{\lambda}{ }^{\omega}$ is derived from Specim AISA "Eagle" pushbroom sensor $(0.40-0.97 \mu \mathrm{m}, 253$ channels) mounted on the Natural Environment Research Council (NERC) Airborne Research and Survey Facility (ARSF) Dornier 228 plane observed on 3 June 2010 (NERC ARSF, 2010). The radiometrically calibrated observations are georeferenced using flight navigation data and orthorectified using on-board Light Detection and Ranging (LiDAR) data with NERC-ARF-DAN (Natural Environmental Research Council Airborne Research Facility Data Analysis Node) APL (Airborne Processing Library) software (NERCARF, 2016) at $1 \mathrm{~m}$ spatial resolution and cropped to the MW area extent. After rejecting shaded pixels and atmospheric absorption bands, $r \lambda^{\omega}$ is classified using $\mathrm{k}$-means $(\mathrm{k}=3)$ clustering (Leutner and Horning, 2016). The three clusters identified are: low reflectance, high reflectance and vegetation. Low reflectance and shaded surface clusters are assigned to "dark" pixels in the $r_{\lambda}{ }^{\omega}$ dataset and high reflectance clusters are assigned to "light" pixels. Google Earth imagery between 2010 and 2017 suggests land cover and surface materials remain largely unchanged within the study area. Processes such as weathering and re-roofing that may impact the classification are not accounted for.

Roof and ground DSM triangles are assigned materials using $r^{\mathrm{LC}}$ and $r_{\lambda}{ }^{\omega}$ based on the intersection of the (X, Y) coordinates of a triangle centroid and the (X, Y) coordinates of each raster cell. Ground surfaces are assigned ground[grass] from $r^{\mathrm{LC}}$. Roof surfaces are assigned Roof[dark] or Roof[light] from $r^{\omega}$. Ground surfaces are not assigned any further material properties as they are often shaded and occluded by trees and buildings when viewed from airborne platforms (Weng, 2012). Materials of vertical surfaces cannot be informed by the plan view raster datasets. As highresolution urban land cover and material datasets are almost universally limited, further land cover classes and surface material classification are challenging to include. 


\section{Appendix C. Thermal camera enclosures}

For this observation campaign new enclosures were designed. Consideration was given to sealed enclosures but as these would require LWIRtransparent windows they were not used as the windows 1) may also be susceptible to the same degradation, and 2) would gradually and unaccountably reduce the transmittance of the camera system. The solution developed protects the lenses without directly interfering with the camera system.

Each enclosure (Spelsberg TK-PS IP66 polystyrene enclosures, $182 \mathrm{~mm}$ x $180 \mathrm{~mm}$ x $111 \mathrm{~mm}$ ) housed a camera, a Raspberry Pi model B computer and a servo motor (Hitec HS-322HD) that controlled an external shutter. Enclosures are covered in reflective aluminium tape to minimise absorption of radiation. The Raspberry Pi controlled the servo motor, the internal shutter motor of the camera, and recorded the internal body temperature sensor of the camera. A shutter mounted on the servo motor occluded an opening in the enclosure located in front of the camera. A schematic for the components used inside each enclosure is shown in Figure S-4 and Table S-1 (Supplement S1).

\section{References}

Adderley, C., Christen, A. and Voogt, J. A. (2015) 'The effect of radiometer placement and view on inferred directional and hemispheric radiometric temperatures of an urban canopy', Atmospheric Measurement Techniques, 8(7), pp. 2699-2714. doi: 10.5194/amt-8-2699-2015.

Agisoft LLC (2013) Agisoft PhotoScan Professional, http://www.agisoft.ru/products/photoscan/professional/. Available at: http://www.agisoft.ru/products/photoscan/professional/ (Accessed: 23 March 2017).

Aoyagi, T. and Takahashi, S. (2012) 'Development of an Urban Multilayer Radiation Scheme and Its Application to the Urban Surface Warming Potential', Boundary-Layer Meteorology, 142(2), pp. 305-328. doi: 10.1007/s10546-011-9679-0.

Becker, F. and Zhao-Liang Li (1995) 'Surface temperature and emissivity at various scales: definition, measurement and related problems', Remote Sensing Reviews, 12(3-4), pp. 225-253. doi: 10.1080/02757259509532286.

Blender (2018) 'Blender 2.79b'. Blender Institute, Amsterdam. Available at: https://www.blender.org/.

Bueno, B. et al. (2012) 'Development and evaluation of a building energy model integrated in the TEB scheme', Geoscientific Model Development, 5(2), pp. 433-448. doi: 10.5194/gmd-5-433-2012.

Chen, F. et al. (2016) 'Effect of emissivity uncertainty on surface temperature retrieval over urban areas: Investigations based on spectral libraries', ISPRS Journal of Photogrammetry and Remote Sensing, 114, pp. 53-65. doi: 10.1016/j.isprsjprs.2016.01.007.

Christen, A., Meier, F. and Scherer, D. (2012) 'High-frequency fluctuations of surface temperatures in an urban environment', Theoretical and Applied Climatology, 108(1-2), pp. 301-324. doi: 10.1007/s00704-011-0521-x.

Chrysoulakis, N. et al. (2018) 'Urban energy exchanges monitoring from space', Scientific Reports, 8(1), p. 11498. doi: 10.1038/s41598-01829873-x.

Crawford, B. et al. (2017) 'Spatial and temporal patterns of surface-atmosphere energy exchange in a dense urban environment using scintillometry', Quarterly Journal of the Royal Meteorological Society, 143(703), pp. 817-833. doi: 10.1002/qj.2967.

Evans, S., Hudson-Smith, A. and Batty, M. (2011) '3-D GIS: Virtual London and beyond', Cybergeo. doi: 10.4000/cybergeo.2871.

Gastellu-Etchegorry, J. P. (2008) '3D modeling of satellite spectral images, radiation budget and energy budget of urban landscapes', Meteorology and Atmospheric Physics, 102(3-4), pp. 187-207. doi: 10.1007/s00703-008-0344-1.

Gastellu-Etchegorry, J. P. et al. (2015) 'Discrete anisotropic radiative transfer (DART 5) for modeling airborne and satellite spectroradiometer and LIDAR acquisitions of natural and urban landscapes', Remote Sensing, 7(2), pp. 1667-1701. doi: 10.3390/rs70201667.

Gastellu-Etchegorry, J. P. et al. (2017) 'DART: Recent advances in remote sensing data modeling with atmosphere, polarization, and chlorophyll fluorescence', IEEE Journal of Selected Topics in Applied Earth Observations and Remote Sensing, 10(6), pp. 2640-2649. doi: 10.1109/JSTARS.2017.2685528.

Gastellu-Etchegorry, J. P., Grau, E. and Lauret, N. (2012) 'DART: A 3D Model for Remote Sensing Images and Radiative Budget of Earth Surfaces', in Modeling and Simulation in Engineering. InTech. doi: 10.5772/31315.

Ghandehari, M., Emig, T. and Aghamohamadnia, M. (2018) 'Surface temperatures in New York City: Geospatial data enables the accurate prediction of radiative heat transfer', Scientific Reports. Springer US, 8(1), pp. 1-10. doi: 10.1038/s41598-018-19846-5.

Ghent, D. et al. (2010) 'Assimilation of land surface temperature into the land surface model JULES with an ensemble Kalman filter', Journal of Geophysical Research Atmospheres, 115(19), pp. 1-16. doi: 10.1029/2010JD014392.

Gong, F. Y. et al. (2018) 'Mapping sky, tree, and building view factors of street canyons in a high-density urban environment', Building and Environment. Elsevier, 134(December 2017), pp. 155-167. doi: 10.1016/j.buildenv.2018.02.042.

Google (2019a) 'Google Earth Pro', Google. Google. Available at: https://www.google.com/intl/en_uk/earth/desktop/.

Google (2019b) Google Maps API. Available at: maps.googleapis.com.

Grau, E. and Gastellu-Etchegorry, J. P. (2013) 'Radiative transfer modeling in the Earth-Atmosphere system with DART model', Remote Sensing of Environment. Elsevier Inc., 139, pp. 149-170. doi: 10.1016/j.rse.2013.07.019.

Grimmond, C. S. B. et al. (2010) 'The International Urban Energy Balance Models Comparison Project: First Results from Phase 1', Journal of Applied Meteorology and Climatology, 49(6), pp. 1268-1292. doi: 10.1175/2010JAMC2354.1.

Gronemeier, T., Raasch, S. and Ng, E. (2017) 'Effects of unstable stratification on ventilation in Hong Kong', Atmosphere, 8(9), pp. 1-15. doi: 10.3390/atmos8090168.

Hammerle, A. et al. (2017) 'Implications of atmospheric conditions for analysis of surface temperature variability derived from landscape-scale thermography', International Journal of Biometeorology. International Journal of Biometeorology, 61(4), pp. 575-588. doi: $10.1007 / \mathrm{s} 00484-016-1234-8$.

Harman, I. N., Best, M. J. and Belcher, S. E. (2004) 'Radiative exchange in an urban street canyon', Boundary-Layer Meteorology, 110(2), pp. 301-316. doi: 10.1023/A:1026029822517.

Harshan, S. et al. (2018) 'Evaluation of an urban land surface scheme over a tropical suburban neighborhood', Theoretical and Applied Climatology. Theoretical and Applied Climatology, 133(3-4), pp. 867-886. doi: 10.1007/s00704-017-2221-7.

Hartley, R. I. and Zisserman, A. (2004) Multiple View Geometry in Computer Vision. Second. Cambridge University Press.

Hénon, A. et al. (2012) 'An urban neighborhood temperature and energy study from the CAPITOUL experiment with the Solene model', Theoretical and Applied Climatology, 110(1-2), pp. 197-208. doi: 10.1007/s00704-012-0616-z.

Hogan, R. J. (2019) 'An Exponential Model of Urban Geometry for Use in Radiative Transfer Applications', Boundary-Layer Meteorology. Springer Netherlands, 170(3), pp. 357-372. doi: 10.1007/s10546-018-0409-8.

Jeanjean, A. P. R. et al. (2017) 'Air quality affected by trees in real street canyons: The case of Marylebone neighbourhood in central London', Urban Forestry \& Urban Greening. Elsevier GmbH., 22, pp. 41-53. doi: 10.1016/j.ufug.2017.01.009.

Jimenez-Munoz, J. C. et al. (2009) 'Revision of the single-channel algorithm for land surface temperature retrieval from landsat thermal-infrared data', IEEE Transactions on Geoscience and Remote Sensing, 47(1), pp. 339-349. doi: 10.1109/TGRS.2008.2007125.

Jiménez-Muñoz, J. C. and Sobrino, J. A. (2006) 'Error sources on the land surface temperature retrieved from thermal infrared single channel 
remote sensing data', International Journal of Remote Sensing, 27(5), pp. 999-1014. doi: 10.1080/01431160500075907.

Kazhdan, M. and Hoppe, H. (2013) 'Screened poisson surface reconstruction', ACM Transactions on Graphics, 32(3), pp. 1-13. doi: $10.1145 / 2487228.2487237$.

Kotthaus, S. et al. (2014) 'Derivation of an urban materials spectral library through emittance and reflectance spectroscopy', ISPRS Journal of Photogrammetry and Remote Sensing. International Society for Photogrammetry and Remote Sensing, Inc. (ISPRS), 94, pp. 194-212. doi: 10.1016/j.isprsjprs.2014.05.005.

Krayenhoff, S. E. and Voogt, J. A. (2007) 'A microscale three-dimensional urban energy balance model for studying surface temperatures', Boundary-Layer Meteorology, 123(3), pp. 433-461. doi: 10.1007/s10546-006-9153-6.

Krayenhoff, S. and Voogt, J. (2016) 'Daytime thermal anisotropy of urban neighbourhoods: Morphological causation', Remote Sensing, 8(2), pp. 1-22. doi: 10.3390/rs8020108.

Kuusk, A. (2017) ‘Canopy Radiative Transfer Modeling', in Comprehensive Remote Sensing. Elsevier, pp. 9-22. doi: 10.1016/b978-0-12409548-9.10534-2.

Lagouarde, J. P. et al. (2010) 'Modelling daytime thermal infrared directional anisotropy over Toulouse city centre', Remote Sensing of Environment, 114(1), pp. 87-105. doi: 10.1016/j.rse.2009.08.012.

Lagouarde, J. P. et al. (2014) 'Directional anisotropy of brightness surface temperature over vineyards: Case study over the Medoc Region (SW France)', IEEE Geoscience and Remote Sensing Letters, 11(2), pp. 574-578. doi: 10.1109/LGRS.2013.2282492.

Lalic, B. and Mihailovic, D. T. (2004) 'An Empirical Relation Describing Leaf-Area Density inside the Forest for Environmental Modeling', Journal of Applied Meteorology, 43(4), pp. 641-645. doi: 10.1175/1520-0450(2004)043<0641:aerdld >2.0.co;2.

Lee, S. et al. (2018) 'Analyzing thermal characteristics of urban streets using a thermal imaging camera: A case study on commercial streets in Seoul, Korea', Sustainability (Switzerland), 10(2), pp. 1-21. doi: 10.3390/su10020519.

Leutner, B. and Horning, N. (2016) 'RStoolbox: Tools for Remote Sensing Data Analysis. R package version 0.1.4.9000'. Available at: https://github.com/bleutner/RStoolbox.

Li, D. and Bou-Zeid, E. (2014) 'Quality and sensitivity of high-resolution numerical simulation of urban heat islands', Environmental Research Letters. IOP Publishing, 9(5). doi: 10.1088/1748-9326/9/5/055001.

Lindberg, F. et al. (2019) 'Exploiting satellite, meteorological and geo-data for urban net storage heat flux modelling.', Theoretical and Applied Climatology, Submitted.

Lindberg, F. and Grimmond, C. S. B. (2011) 'Nature of vegetation and building morphology characteristics across a city: Influence on shadow patterns and mean radiant temperatures in London', Urban Ecosystems, 14(4), pp. 617-634. doi: 10.1007/s11252-011-0184-5.

Masson, V. (2000) 'A physically-based scheme for the urban energy budget in atmospheric models', Boundary-Layer Meteorology, 94(3), pp. 357-397. doi: 10.1023/A:1002463829265.

Meier, F. et al. (2011) 'Atmospheric correction of thermal-infrared imagery of the 3-D urban environment acquired in oblique viewing geometry’, Atmospheric Measurement Techniques, 4(5), pp. 909-922. doi: 10.5194/amt-4-909-2011.

Meier, F. and Scherer, D. (2012) 'Spatial and temporal variability of urban tree canopy temperature during summer 2010 in Berlin, Germany', Theoretical and Applied Climatology, 110(3), pp. 373-384. doi: 10.1007/s00704-012-0631-0.

Meier, F., Scherer, D. and Richters, J. (2010) 'Determination of persistence effects in spatio-temporal patterns of upward long-wave radiation flux density from an urban courtyard by means of Time-Sequential Thermography', Remote Sensing of Environment. Elsevier Inc., 114(1), pp. 21-34. doi: 10.1016/j.rse.2009.08.002.

Mitraka, Z. et al. (2012) 'Improving the estimation of urban surface emissivity based on sub-pixel classification of high resolution satellite imagery', Remote Sensing of Environment. Elsevier Inc., 117, pp. 125-134. doi: 10.1016/j.rse.2011.06.025.

Moritz, S. and Bartz-Beielstein, T. (2017) ‘imputeTS: Time Series Missing Value Imputation in R', The R Journal, 9(1), pp. 207-218. Available at: https://journal.r-project.org/archive/2017/RJ-2017-009/index.html.

Morrison, W. et al. (2018) 'A novel method to obtain three-dimensional urban surface temperature from ground-based thermography', Remote Sensing of Environment. Elsevier, 215(May), pp. 268-283. doi: 10.1016/j.rse.2018.05.004.

Morrison, W. T. J. (2019) 'Atmospheric and emissivity corrections for ground-based thermography using 3D radiative transfer modelling supplementary data'. doi: 10.5281/zenodo.3458049.

Morrison, W. T. J. and Yin, T. (2019) 'DART-atmos-corr', GitHub repository. GitHub. Available at: https://github.com/Urban-MeteorologyReading/DART-atmos-corr.

NERC-ARF (2016) NERC Airborne Research Facility Data Analysis Node, Software Downloads. Available at: https://nerc-arfdan.pml.ac.uk/trac/wiki/Downloads/software (Accessed: 25 July 2017).

NERC ARSF (2010) 'Data from the Photographic Camera, ATM AZ-16, Optech LIDAR and AISA Eagle and Hawk Instruments on-board the Dornier Do228-101 D-CALM Aircraft during Flight GB08/19 over London Area.' NERC. Available at: https://catalogue.ceda.ac.uk/uuid/d0d41776042b3d81651afldafd2f883f.

Nunez, M., Eliasson, I. and Lindgren, J. (2000) 'Spatial variation of incoming longwave radiation in Göteborg, Sweden', Theoretical and Applied Climatology, 67(3-4), pp. 181-192. doi: 10.1007/s007040070007.

Offerle, B. et al. (2007) 'Surface heating in relation to air temperature, wind and turbulence in an urban street canyon', Boundary-Layer Meteorology, 122(2), pp. 273-292. doi: 10.1007/s10546-006-9099-8.

Optris GmbH (2018) 'Optris PI Operator's Manual'. Germany. Available at: https://www.optris.co.uk/thermal-imager-pi160.

Ordnance Survey (2018) 'OS MasterMap Greenspace Layer'. Ordnance Survey Limited. Available at: https://www.ordnancesurvey.co.uk/business-and-government/products/os-mastermap-greenspace.html.

Pigeon, G. et al. (2008) 'Simulation of fall and winter surface energy balance over a dense urban area using the TEB scheme', Meteorology and Atmospheric Physics, 102(3-4), pp. 159-171. doi: 10.1007/s00703-008-0320-9.

Pisek, J., Ryu, Y. and Alikas, K. (2011) 'Estimating leaf inclination and G-function from leveled digital camera photography in broadleaf canopies', Trees - Structure and Function, 25(5), pp. 919-924. doi: 10.1007/s00468-011-0566-6.

Plowright, A. (2018) 'ForestTools: Analyzing Remotely Sensed Forest Data v.0.2'. Available at: https://cran.r-project.org/package=ForestTools.

Porson, A. et al. (2010) 'Implementation of a new urban energy budget scheme in the MetUM. Part I: Description and idealized simulations', Quarterly Journal of the Royal Meteorological Society, 136(651), pp. 1514-1529. doi: 10.1002/qj.668.

Qi, J. et al. (2019) 'LESS: LargE-Scale remote sensing data and image simulation framework over heterogeneous 3D scenes', Remote Sensing of Environment. Elsevier, 221(March 2018), pp. 695-706. doi: 10.1016/j.rse.2018.11.036.

Rotach, M. W. et al. (2005) 'BUBBLE - An urban boundary layer meteorology project', Theoretical and Applied Climatology, 81(3-4), pp. 231-261. doi: 10.1007/s00704-004-0117-9.

Roussel, J.-R. and Auty, D. (2018) 'lidR: Airborne LiDAR Data Manipulation and Visualization for Forestry Applications'. Available at: https://cran.r-project.org/package=lidR.

Sobrino, J. A. et al. (2011) 'Evaluation of the DART 3D model in the thermal domain using satellite/airborne imagery and ground-based measurements', International Journal of Remote Sensing, 32(22), pp. 7453-7477. doi: 10.1080/01431161.2010.524672. 
Sobrino, J. A., Coll, C. and Caselles, V. (1991) 'Atmospheric correction for land surface temperature using NOAA-11 AVHRR channels 4 and 5', Remote Sensing of Environment, 38(1), pp. 19-34. doi: 10.1016/0034-4257(91)90069-I.

Sobrino, J. A. and Cuenca, J. (1999) 'Angular variation of thermal infrared emissivity for some natural surfaces from experimental measurements.', Applied optics, 38(18), pp. 3931-6. Available at: http://www.ncbi.nlm.nih.gov/pubmed/18320001.

Verseghy, D. L. and Munro, D. S. (1989) 'Sensitivity studies on the calculation of the radiation balance of urban surfaces: II. Longwave radiation', Boundary-Layer Meteorology, 48(1-2), pp. 1-18. doi: 10.1007/BF00121780.

Vollmer, M. and Möllmann, K.-P. (2017) Infrared Thermal Imaging. Weinheim, Germany: Wiley-VCH Verlag GmbH \& Co. KGaA. doi: $10.1002 / 9783527693306$.

Voogt, J. A. and Grimmond, C. S. B. (2000) 'Modeling Surface Sensible Heat Flux Using Surface Radiative Temperatures in a Simple Urban Area', Journal of Applied Meteorology, 39(10), pp. 1679-1699. doi: 10.1175/1520-0450-39.10.1679.

Voogt, J. a. and Oke, T. R. (1997) 'Complete Urban Surface Temperatures', Journal of Applied Meteorology, 36(9), pp. 1117-1132. doi: 10.1175/1520-0450(1997)036<1117:CUST>2.0.CO;2.

Voogt, J. A. and Oke, T. R. (2003) 'Thermal remote sensing of urban climates', Remote Sensing of Environment, 86(3), pp. 370-384. doi: 10.1016/S0034-4257(03)00079-8.

Wan, Z. (2014) 'New refinements and validation of the collection-6 MODIS land-surface temperature/emissivity product', Remote Sensing of Environment, 140, pp. 36-45. doi: 10.1016/j.rse.2013.08.027.

Wang, D. et al. (2018) 'A Geometric Model to Simulate Urban Thermal Anisotropy for Simplified Neighborhoods', IEEE Transactions on Geoscience and Remote Sensing. IEEE, 56(8), pp. 4930-4944. doi: 10.1109/TGRS.2018.2842794.

Wang, D., Chen, Y. and Zhan, W. (2018) 'A geometric model to simulate thermal anisotropy over a sparse urban surface (GUTA-sparse)', Remote Sensing of Environment. Elsevier, 209(19), pp. 263-274. doi: 10.1016/j.rse.2018.02.051.

Wang, W. M., Li, Z. L. and Su, H. B. (2007) 'Comparison of leaf angle distribution functions: Effects on extinction coefficient and fraction of sunlit foliage', Agricultural and Forest Meteorology, 143(1-2), pp. 106-122. doi: 10.1016/j.agrformet.2006.12.003.

Weng, Q. (2012) 'Remote sensing of impervious surfaces in the urban areas: Requirements, methods, and trends', Remote Sensing of Environment. Elsevier Inc., 117, pp. 34-49. doi: 10.1016/j.rse.2011.02.030.

Wu, S. et al. (2018) 'Characterization of Remote Sensing Albedo Over Sloped Surfaces Based on DART Simulations and In Situ Observations', Journal of Geophysical Research: Atmospheres, 123(16), pp. 8599-8622. doi: 10.1029/2018JD028283.

Yang, J. et al. (2015) 'Modeling the effective emissivity of the urban canopy using sky view factor', ISPRS Journal of Photogrammetry and Remote Sensing. International Society for Photogrammetry and Remote Sensing, Inc. (ISPRS), 105, pp. 211-219. doi: 10.1016/j.isprsjprs.2015.04.006.

Yang, J. et al. (2016) 'Development of an improved urban emissivity model based on sky view factor for retrieving effective emissivity and surface temperature over urban areas', ISPRS Journal of Photogrammetry and Remote Sensing. International Society for Photogrammetry and Remote Sensing, Inc. (ISPRS), 122, pp. 30-40. doi: 10.1016/j.isprsjprs.2016.09.007.

Yang, L. and Li, Y. (2009) 'City ventilation of Hong Kong at no-wind conditions', Atmospheric Environment. Elsevier Ltd, 43(19), pp. 31113121. doi: 10.1016/j.atmosenv.2009.02.062.

Yin, T. et al. (2013) 'A new approach of direction discretization and oversampling for 3D anisotropic radiative transfer modeling', Remote Sensing of Environment. Elsevier Inc., 135, pp. 213-223. doi: 10.1016/j.rse.2013.03.030.

Yin, T., Lauret, N. and Gastellu-Etchegorry, J. P. (2015a) 'Simulating images of passive sensors with finite field of view by coupling 3-D radiative transfer model and sensor perspective projection', Remote Sensing of Environment. Elsevier Inc., 162, pp. 169-185. doi: 10.1016/j.rse.2015.02.020.

Yin, T., Lauret, N. and Gastellu-Etchegorry, J. P. (2015b) 'Simulating images of passive sensors with finite field of view by coupling 3-D radiative transfer model and sensor perspective projection', Remote Sensing of Environment. Elsevier Inc., 162, pp. 169-185. doi: 10.1016/j.rse.2015.02.020.

Zeng, L. et al. (2018) 'A fast approach for large-scale Sky View Factor estimation using street view images', Building and Environment. Elsevier, 135(December 2017), pp. 74-84. doi: 10.1016/j.buildenv.2018.03.009. 


\section{S1. Thermal camera enclosures}

When the LWIR cameras (Optris PI-160) were installed in central London (Figure S-1) during 2015-16, damage occurred from exposure despite being advertised as having complete protection against both dust contact and water immersion up to $1 \mathrm{~m}$ depth ("IP67" rating from IEC 60529 international standard). The original aluminium enclosures covered the top of each camera (Figure S-1) to limit differential and rapid warming of the camera body from direct sunlight.
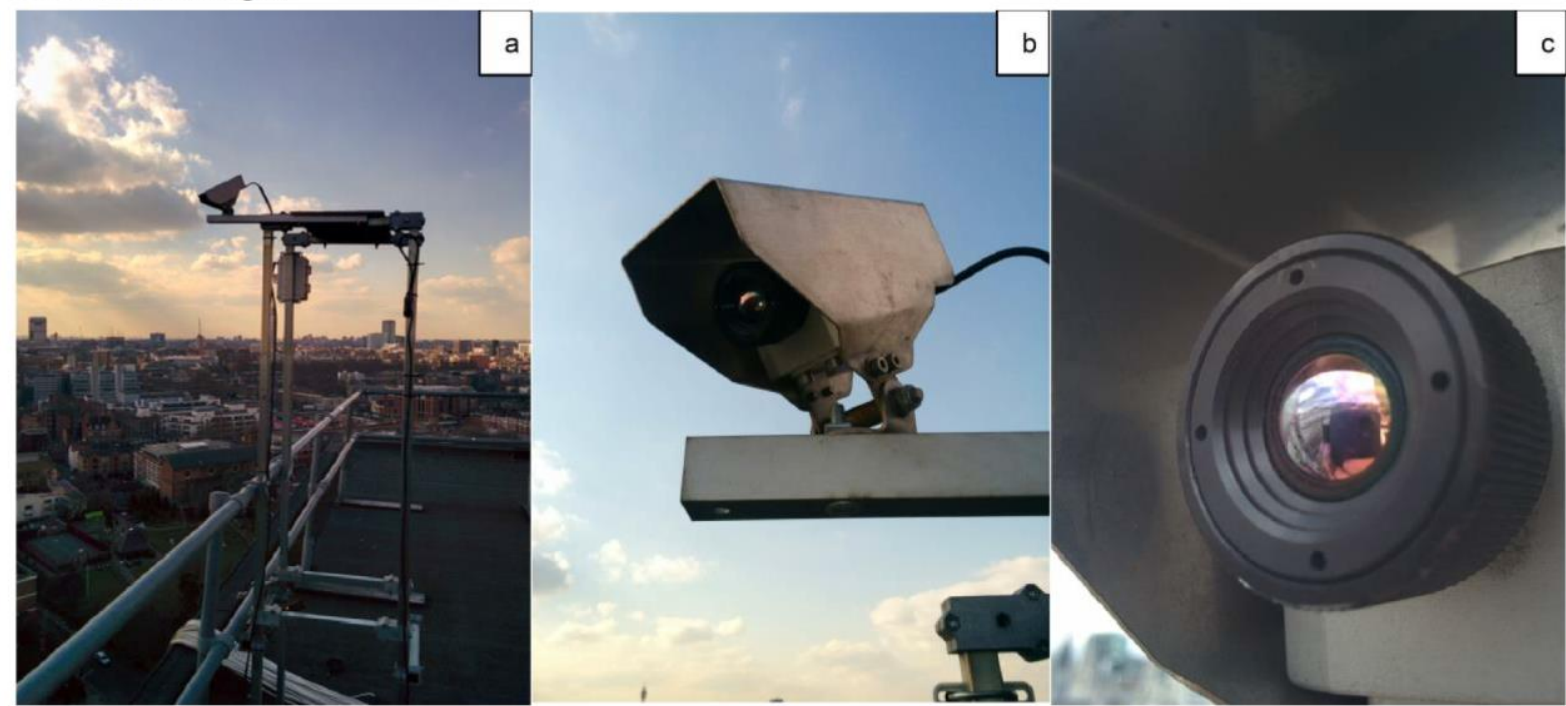

Figure S-1. Example of earlier (2015-16) setup of Optris PI-160 LWIR camera at the IMU site with (a) overall mounting of south facing camera (photo looking southwest), (b) close-up of the Optris PI-160 camera in original aluminium housing, and (c) front of the camera within the aluminium housing.

Examples of the degradation of the lens anti-reflective coating include a sensor installed at $110 \mathrm{~m}$ residential tower facing into the prevailing winds (south-westerly) for 11 months (Figure S-2b, right). The degradation is evident when compared to a new lens (e.g. Figure S-2a, left; Figure S-2b, left). Unfortunately, the overall effect of the degradation is an unaccountable reduction in transmissivity of the lens over time. This reduction in observational accuracy made images appear to be out of focus (image aberrations). Attempts to clean the lenses during and after the 2015-16 campaign concluded the lenses were damaged beyond repair.

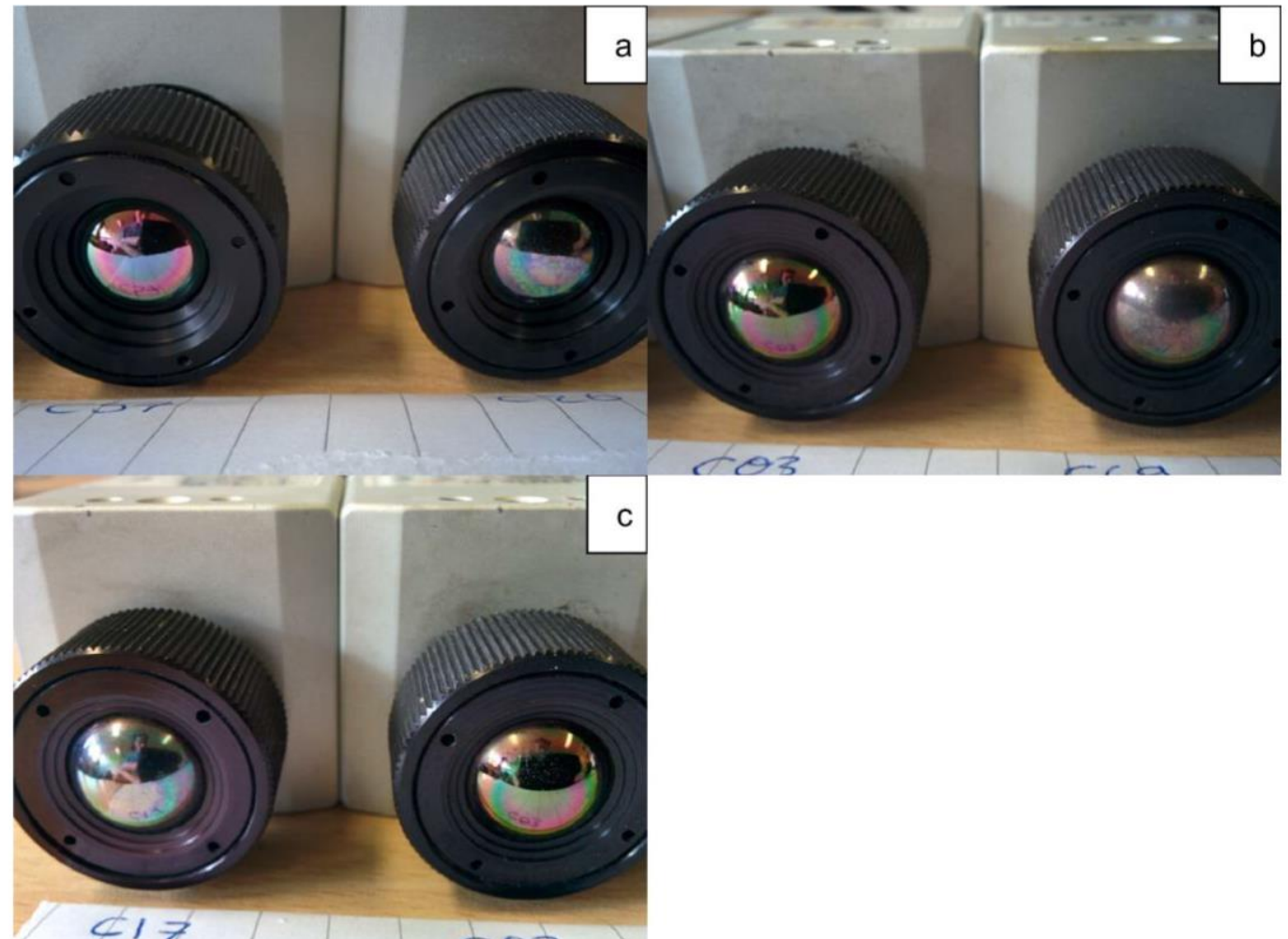

Figure S-2. Camera lenses after the 2015-16 observation campaign, camera body serial numbers ending in (a) 57 and 26 (b) 03 and 69 (c) 17 and 03. 


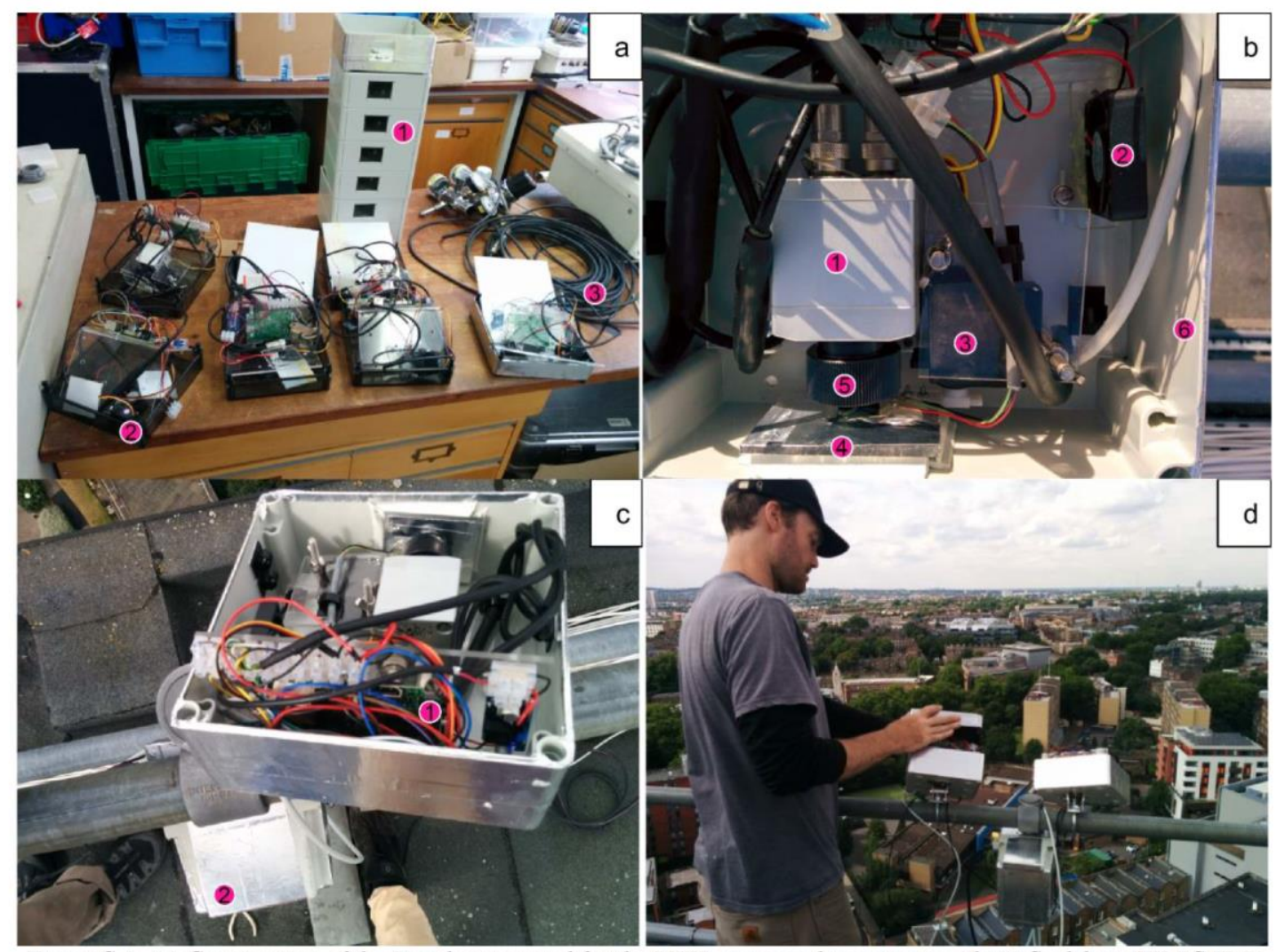

Figure S-3. (a) Construction of the 7 enclosures used for the LWIR camera observations with (1) Spelsberg TK-PS IP66 polystyrene, $182 \mathrm{~mm} \times 180 \mathrm{~mm} \times 111 \mathrm{~mm}$ enclosure housings without aluminium tape, (2) lids for the enclosure housings prior to aluminium tape application and temporarily holding the electronics for each enclosure, (3) $20 \mathrm{~m}$ USB data transfer cable between the camera and data collection computer, (b) completed enclosure with (1) Optris PI-160 LWIR camera, (2) $30 \mathrm{~mm}$ fan with $5 \mathrm{~mm}$ holes underneath to promote the circulation of air within the enclosures, (3) Hitec HS-322HD servo motor attached to (4) a shutter that covers the opening in the enclosure, (5) camera lens assembly (6) enclosure housing, (c) enclosure installed at the IMU site with the camera facing northeast with (1) the Raspberry Pi and electronics housed at the back of the enclosure on an L-shaped acrylic plate, (2) additional housing for an ethernet switch and $12 \mathrm{~V}$ power supply, (d) final installation of the camera (shown in c) with Dr Ben Crawford closing lid.

Table S-1. Description of process interface (PIF) connectors for Optris PI-160 cameras (Optris GmbH, 2018), referenced numerically in Figure $S-4$.

\begin{tabular}{|c|c|c|}
\hline PIF port \# (Figure S-4) & Short name & Description \\
\hline $\mathbf{1}$ & PIF_VIN & Power: 5V DC +ve \\
\hline $\mathbf{2}$ & Shield & - \\
\hline $\mathbf{3}$ & PIF_AO1 & Analog output \\
\hline $\mathbf{4}$ & PIF_AIN1 & Analog input \\
\hline $\mathbf{5}$ & PIF_DIN1 & Digital input \\
\hline $\mathbf{6}$ & PIF_GND & Ground \\
\hline
\end{tabular}




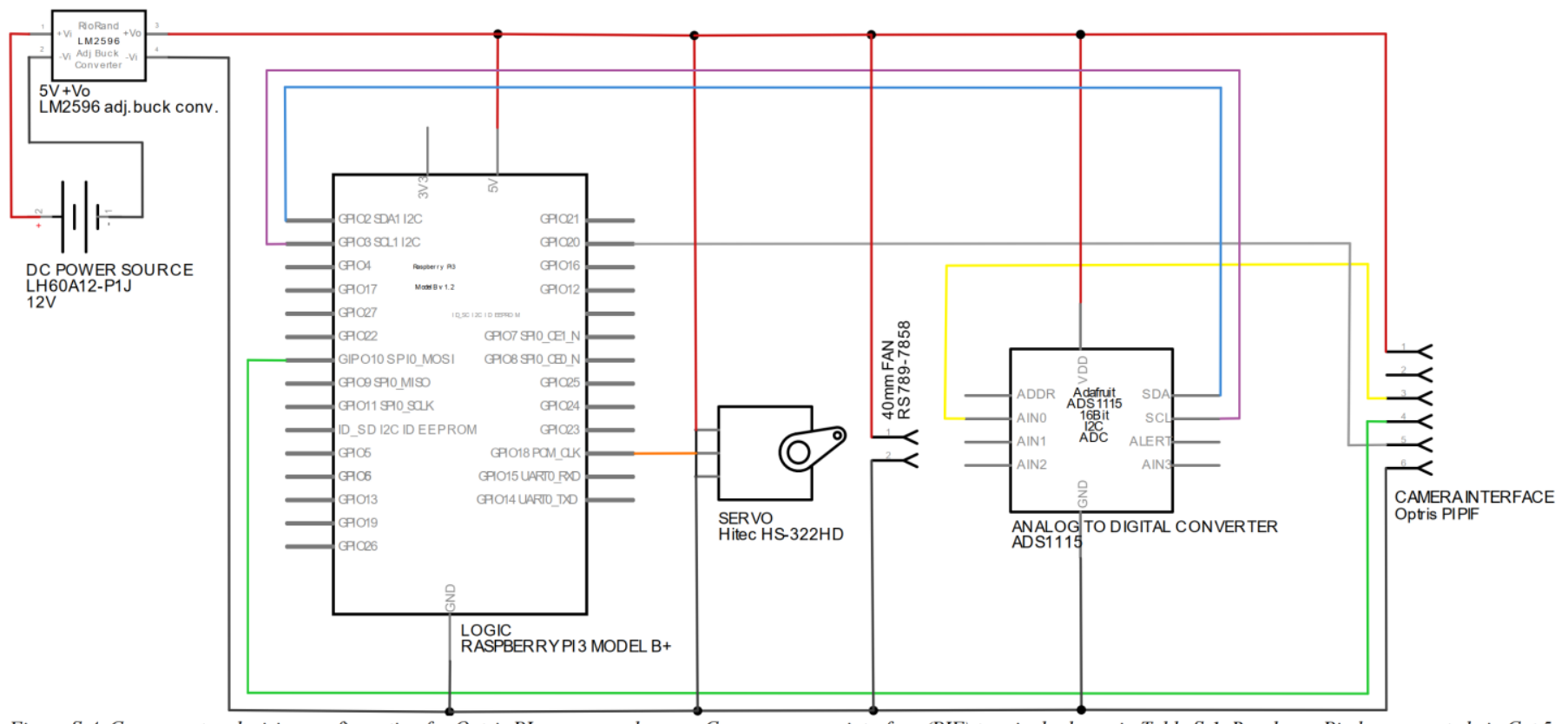

Figure S-4. Component and wiring configuration for Optris PI camera enclosures. Camera process interface (PIF) terminals shown in Table S-1. Raspberry Pi also connected via Cat 5 Ethernet to give an internet connection over a local area network.

\section{S2. Camera spectral response function}

As the spectral response function (SRF) for the cameras is unavailable from the manufacturer except for a generic focal plane array (FPA) detector response function, the full camera response function that includes the FPA and lens is derived from laboratory experiments for camera $\mathrm{C} 2$ from $6-14 \mu \mathrm{m}$ at $0.1 \mu \mathrm{m}$ resolution using a Bentham TMS300 single monochromator emission source, with reference measurements of the emission source using a Bentham Py-CAL pyroelectric detector. The emission source is sampled by $\mathrm{C} 2$ using the camera digital number (DN) output across a $9 \times 3$ region of pixels that observe the centre of the projected emission source at the monochromator exit slit. The per-pixel DN values are normalised and the median of this is compared to the normalised signal from the Py-CAL detector which has a flat response function across the entire bandwidth. From the two normalised responses of the emission source across the spectral range, the SRF was determined and applied across each pixel of each camera.

\section{S3. Siting of camera for observational evaluation of atmospheric correction}

A longwave infrared camera (C7) was installed at the atmospheric correction evaluation site ("CUB" site). The flat asphalt felt roof $\left(\sim 600 \mathrm{~m}^{2}\right)$ is surrounded by a low $(<2 \mathrm{~m})$ metal railing and connected by a metal stairway.

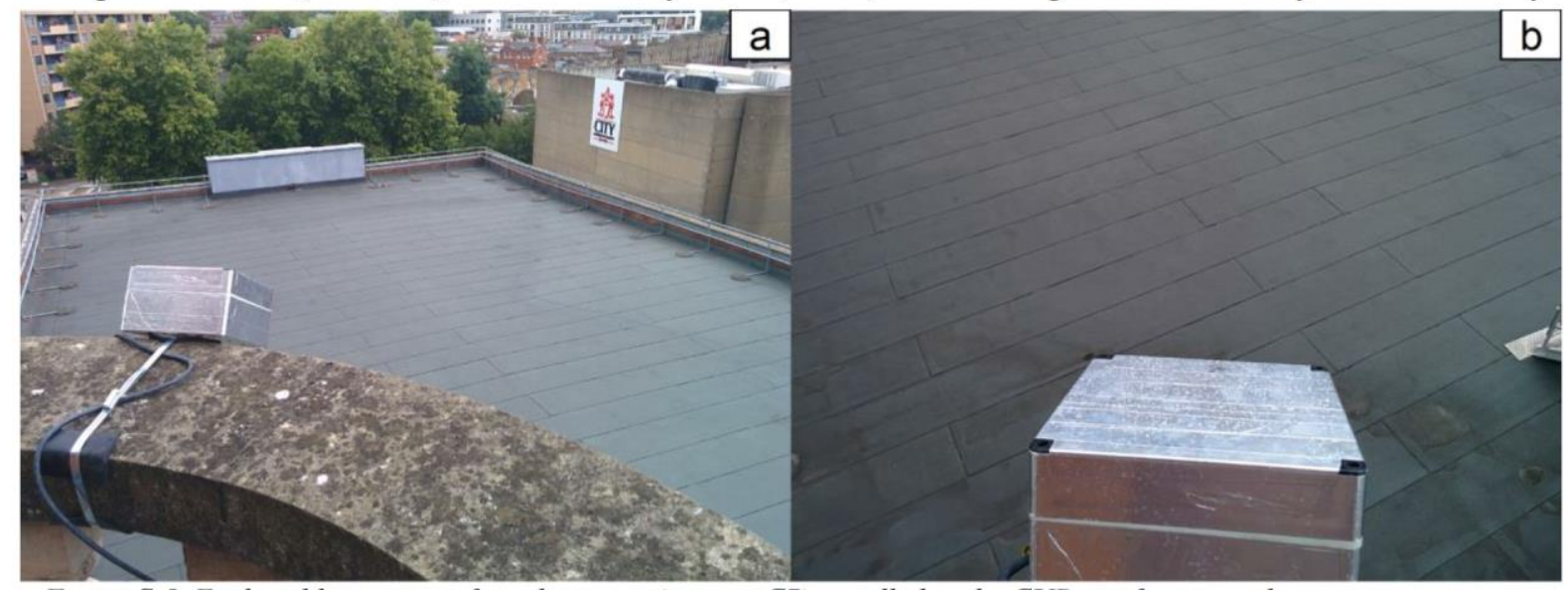

Figure S-5. Enclosed longwave infrared camera (camera C7) installed at the CUB site for atmospheric correction evaluation with its "short" roof-camera path length (a) mounted on a bell tower to the southwest of the target roof. Images 7 September 2017.

A bell tower ( $\sim 12 \mathrm{~m}$ taller than the roof) to the southwest of the roof was used to mount the enclosed (Supplement S2) camera at $\sim 6.5 \mathrm{~m}$ above the roof facing northeast and pointing towards the flat roof below. To the east and west of the roof, there are no taller buildings in proximity $(\sim 100 \mathrm{~m})$, allowing the camera target area in the centre of the roof to be mostly sunlit even during low sun angles around sunrise and sunset. 


\section{S4. Statistics for atmosphere correction}

(a)

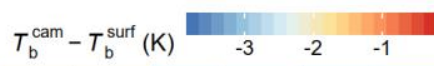
\begin{tabular}{ll|l|l|l|l|l|}
$\mathbf{2 5 0}$ & $-2.07[1.29]$ & $-2.06[1.31]$ & $-338[0.88]$ & $-2.79[1.34]$ & $-3.48[207]$ & $-376[270]$ \\
\hline
\end{tabular} \begin{tabular}{|l|l|l|l|l|l|l|}
\hline 200 & $-1.27[0.64]$ & $-1.25[0.73]$ & $-1.94[0.72]$ & $-1.74[0.77]$ & $-1.88[0.87]$ & $-1.96[1.00]$ \\
\hline
\end{tabular} \begin{tabular}{lll|l|l|l|l|l|}
\hline $\mathbf{5}$ & $\mathbf{1 5 0}$ & $-0.72[0.30]$ & $-0.67[0.36]$ & $-1.33[0.77]$ & $-1.14[0.53]$ & $-1.10[0.43]$ & $-1.05[0.47]$ \\
\hline
\end{tabular}

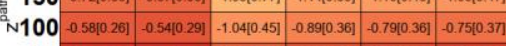
50

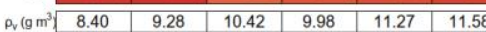

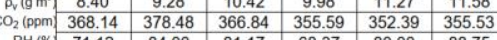
\begin{tabular}{c|c|c|c|c|c|c|c|}
$\mathrm{RH}(\%)$ & 71.12 & 84.00 & 81.17 & 68.37 & 80.00 & 88.75 \\
\hline$T_{\mathrm{a}}(\mathrm{K})$ & 286.85 & 285.75 & 288.20 & 290.33 & 28974 & 288.47 \\
\cline { 2 - 7 } & 02.00 & $06: 00$ & $10: 00$ & 14.00 & $18: 00$ & $22: 00$ \\
\hline
\end{tabular} 02:00 06:00 10:00 14:00 18:00 22:00 2017-09-07 (HH:MM) (b) $L^{\text {atm }}\left(\mathrm{W} \mathrm{m}^{-2} \mathrm{sr}^{-1}\right) \quad 2 \quad 3 \quad 4 \quad 5$

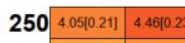

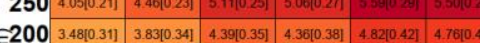
\begin{tabular}{lllllllll}
150 & $2.82[0.23]$ & $3.10[0.26]$ & $3.55[0.29]$ & $3.540 .29]$ & $3.91[0.32]$ & $3.86[0.32]$ \\
\hline
\end{tabular} \begin{tabular}{|l|l|l|l|l|l|l|}
\hline N100 & $1.98[0.20]$ & $2.17[0.22]$ & $249[0.25]$ & $249[0.25]$ & $2.75[0.28]$ & $271[0.28]$ \\
\hline
\end{tabular} \begin{tabular}{lll|l|l|l|l|l|l|}
$\mathbf{5 0}$ & $1.41[0.36]$ & $1.53[0.39]$ & $1.78[0.45]$ & $1.78[0.45]$ & $1.96[0.50]$ & $1.92[0.49$ \\
\hline
\end{tabular}

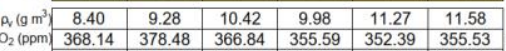
\begin{tabular}{|c|c|c|c|c|c|c|}
\hline $\mathrm{O}_{2}$ (ppm) & 368.14 & 378.48 & 366.84 & 355.59 & 352.39 & 355.53 \\
\hline $\mathrm{RH}(\%)$ & 71.12 & 84.00 & 81.17 & 68.37 & 80.00 & 88.75 \\
\hline
\end{tabular} 02:00 06:00 10:00 14:00 18:00 22:00 2017-09-07 (HH:MM) (c) $\Gamma^{\text {atm }} \begin{array}{llllll}0.84 & 0.87 & 0.90 & 0.93 & 0.96\end{array}$

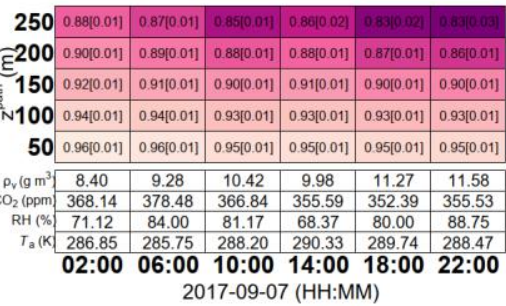

Figure S-6. Atmospheric correction of longwave infrared (LWIR) camera observations for six timesteps on 7 September 2017, using pixels within $\pm 10 \mathrm{~m}$ of five path lengths $\left(z^{\text {path }}\right.$ ) with (white cells) meteorological input variables (coloured cells) median [IQR] values of: (a) difference between uncorrected camera brightness temperature $\left(T_{b}{ }^{\text {cam }}\right)$ and corrected surface brightness temperature $\left(T_{b}{ }^{\text {surf }}\right)$, (b) surface-camera path contribution of the at-sensor band integrated atmosphere radiance $\left(L^{\text {atm }}\right)$, and (c) surface-camera path contribution of band integrated atmospheric transmissivity $\left(T^{\text {atm }}\right)$. See list of symbols and acronyms in main article for all other definitions. See Section 3 in main article for details of calculation methods.

(a) $T_{\mathrm{b}}^{\mathrm{cam}}-T_{\mathrm{b}}^{\text {surf }}(\mathrm{K})$

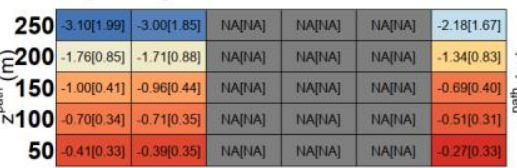

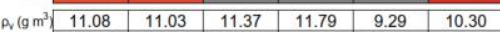
\begin{tabular}{c|c|c|c|c|c|c|c|}
\hline RH (\%) & 356.11 & 450.51 & 373.14 & 376.76 & 351.91 & 373.51 \\
\hline & 85.00 & 91.00 & 88.00 & 89.35 & 75.85 & 84.00 \\
\hline
\end{tabular}

\begin{tabular}{l|l|l|l|l|l|l|}
$T_{\mathrm{a}}(\mathrm{K})$ & 288.45 & 287.27 & 288.31 & 288.66 & 287.43 & 287.45 \\
\hline
\end{tabular} 02:00 06:00 10:00 14:00 18:00 22:00 2017-09-08 (HH:MM) (b)

$L^{\mathrm{atm}}\left(\mathrm{W} \mathrm{m}^{-2} \mathrm{sr}^{-1}\right) \quad 2 \quad 3 \quad 4 \quad 5$

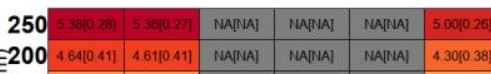

\begin{tabular}{|l|l|l|l|l|l|l|}
\hline $\mathbf{1 5 0}$ & $3.76[0.31]$ & $3.74[0.31]$ & Ma[NA] & NATMA] & MATMA] & $3.48[0.29]$ \\
\hline
\end{tabular}

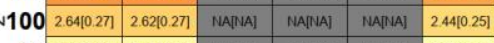

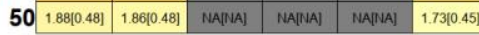

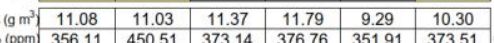
\begin{tabular}{|c|c|c|c|c|c|c|}
\hline RH (\%) & 35.00 & 91.00 & 88.00 & 89.35 & 75.85 & 84.00 \\
\hline
\end{tabular} 02:00 06:00 10:00 14:00 18:00 22:00 2017-09-08 (HH:MM)

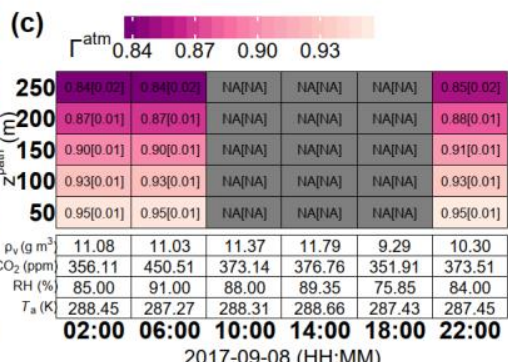

Figure S-7. As Figure S-6 but for 8 September 2017

(a)

250 -1.93[1.40] $-1.95[1.19]$

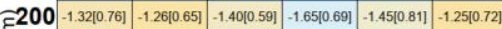

\begin{tabular}{ll|lll|l|l|l|}
\hline 5 & 150 & $-0.68[0.47]$ & $-0.73[0.31]$ & $-0.98[0.62]$ & $-1.12[0.55]$ & $-0.81[0.40]$ & $-0.69[0.37]$ \\
\hline
\end{tabular}

\begin{tabular}{llllllll} 
& $N 100$ & $-0.55[0.24]$ & $-0.56[0.26]$ & $-0.75[0.42]$ & $-0.89[0.36]$ & $-0.65[0.31]$ & $-0.56[0.28]$ \\
\hline
\end{tabular}

50

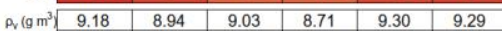

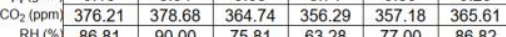

\begin{tabular}{c|c|c|c|c|c|c|c|}
\hline$R H(\%)$ & 86.81 & 90.00 & 75.81 & 63.28 & 77.00 & 86.82 \\
$T_{a}(K)$ & 285.05 & 284.05 & 286.97 & 289.35 & 287.21 & 285.25 \\
\hline
\end{tabular}

02:00 06:00 10:00 14:00 18:00 22:00 2017-09-09 (HH:MM)
$\begin{array}{lllll}T_{\mathrm{b}}^{\mathrm{cam}}-T_{\mathrm{b}}^{\text {surf }}(\mathrm{K})-2.5 & -2.0 & -1.5 & -1.0 & -0.5\end{array}$

(b)

$L^{\mathrm{atm}}\left(\mathrm{W} \mathrm{m}^{-2} \mathrm{sr}^{-1}\right) 1.52 .02 .53 .03 .54 .04 .5$

\section{0}

150 2091 19 2 (2000

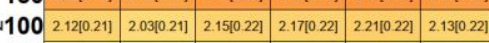
\begin{tabular}{|l|l|l|l|l|l|l|}
50 & $1.49[0.38]$ & $1.43[0.37]$ & $1.54[0.39]$ & $1.54[0.39]$ & $1.57[0.40]$ & $1.51[0.39]$ \\
\hline
\end{tabular}

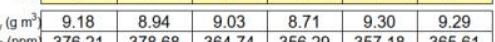

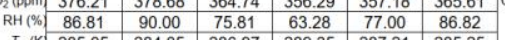
02:00 06:00 10:00 14:00 18:00 22:00 2017-09-09 (HH:MM)

Figure S-8. As Figure S-6 but for 9 September 2017

(a)

$T_{\mathrm{b}}^{\text {cam }}-T_{\mathrm{b}}^{\text {surf }}(\mathrm{K}) \quad-3 \quad-2 \quad-1$

(b)

$L^{\text {atm }}\left(\mathrm{W} \mathrm{m}^{-2} \mathrm{sr}^{-1}\right) 1.5 \quad 2.5 \quad 3.5 \quad 4.5 \quad 5.5$

\begin{tabular}{ll|l|l|l|l|l|l|}
250 & $-1.92[1.26]$ & $-1.88[1.22]$ & $-2790.67]$ & $-2.44[1.20]$ & $-3.32[212]$ & $-1.98[1.35]$ \\
\hline
\end{tabular}

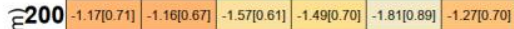

\begin{tabular}{ll|l|l|l|l|l|l|}
\hline & 150 & $-0.64[0.34]$ & $-0.64[0.33]$ & $-1.12[0.70]$ & $-0.97[0.43]$ & $-1.02[0.45]$ & $-0.72[0.33]$ \\
\hline
\end{tabular}

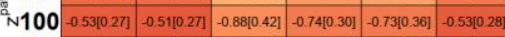

50

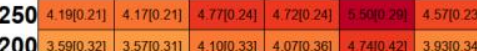

\begin{tabular}{|l|l|l|l|l|l|l|l|}
150 & $2.890 .24]$ & $2.88[0.24]$ & $3.32[0.27]$ & $3.30[0.27]$ & $3.84[0.31]$ & $3.18[0.26]$ \\
\hline
\end{tabular}

\begin{tabular}{|l|l|l|l|l|l|l|}
\hline 100 & $2.02[0.21]$ & $2.0110 .21]$ & $2.33[0.24]$ & $2.32[0.23]$ & $2.70[0.27]$ & $2.23[0.23$ \\
\hline
\end{tabular}

\begin{tabular}{|l|l|l|l|l|l|l|}
$\mathbf{5 0}$ & $1.42[0.37]$ & $1.41[0.37]$ & $1.66[0.42]$ & $1.65[0.42]$ & $1.91[0.49]$ & $1.57[0.41]$ \\
\hline
\end{tabular}

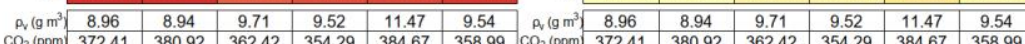

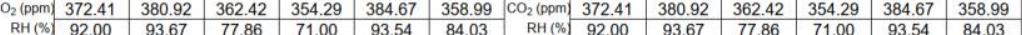

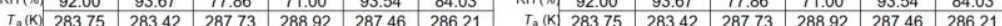
02:00 06:00 10:00 14:00 18:00 22:00 2017-09-10 (HH:MM)

02:00 06:00 2017-09-10 (HH:MM)

Figure S-9. As Figure S-6 but for 10 September 2017

(a)

$T_{\mathrm{b}}^{\text {cam }}-T_{\mathrm{b}}^{\text {surf }}(\mathrm{K}) \quad-2.5 \quad-2.0 \quad-1.5 \quad-1.0 \quad-0.5$

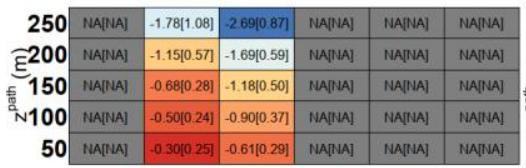

(b)

$L^{\mathrm{atm}}\left(\mathrm{W} \mathrm{m}^{-2} \mathrm{sr}^{-1}\right) \quad 2 \quad 3 \quad 4$

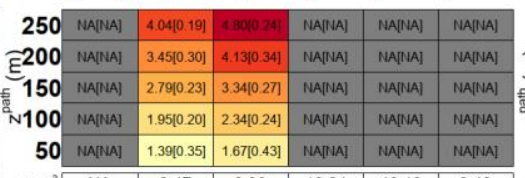

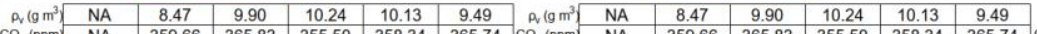

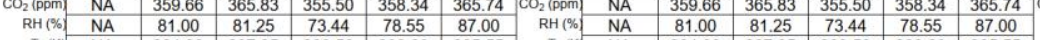
02:00 06:00 10:00 14:00 18:00 22:00 2017-09-11 (HH:MM)
02:00 06:00 10:00 14:00 18:00 22:00 2017-09-11 (HH:MM) 2017-09-08 (HH:MM) (c)

$\begin{array}{llll}0.88 & 0.90 & 0.92 & 0.94\end{array}$

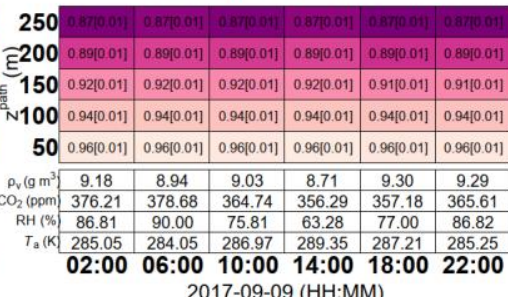

(c) 2017-09-09 (HH:MM)

\begin{tabular}{|l|l|l|l|l|l|l|}
250 & $0.87[0.01]$ & $0.87[0.01]$ & $0.86[0.01]$ & $0.86[0.01]$ & 0.890091 & $0.8710 .01]$ \\
\hline
\end{tabular}

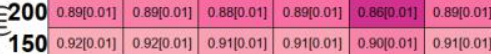

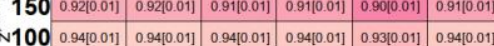
\begin{tabular}{|l|l|l|l|l|l|l|}
50 & $0.96[0.01]$ & $0.96[0.01]$ & $0.95[0.01]$ & $0.96[0.01]$ & $0.95[0.01]$ & $0.96[0.01]$ \\
\hline
\end{tabular}

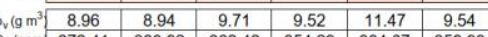
\begin{tabular}{|c|c|c|c|c|c|c|}
\hline R (ppm & 372.41 & 380.92 & 362.42 & 354.29 & 384.67 & 358.99 \\
\hline R $\%$ & 92.00 & 93.67 & 77.86 & 71.00 & 93.54 & 84.03 \\
\hline
\end{tabular} 2017-09-10 (HH:MM)

(c) $\Gamma^{\mathrm{atm}} 0.860 .880 .900 .920 .940 .96$

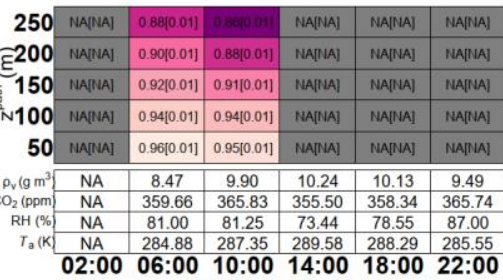

02:00 06:00 10:00 14:00 18:00 $22: 00$ 2017-09-11 (HH:MM)

Figure S-10. As Figure S-6 but for 11 September 2017 
(a)

$T_{\mathrm{b}}^{\mathrm{cam}}-T_{\mathrm{b}}^{\text {surf }}(\mathrm{K}) \quad-2.0 \quad-1.5 \quad-1.0 \quad-0.5$

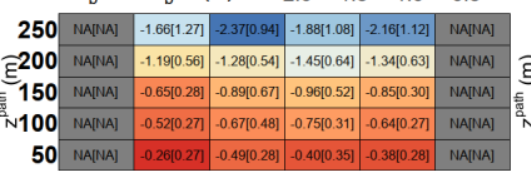

(b) $L^{\mathrm{atm}}\left(\mathrm{W} \mathrm{m}^{-2} \mathrm{sr}^{-1}\right) 1.52025303 .540$

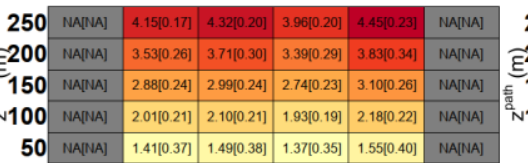

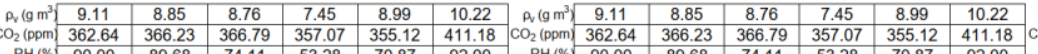

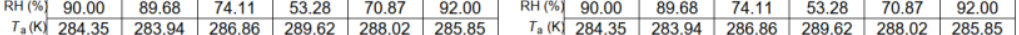
$\begin{array}{llllllllllllll}02: 00 & 06: 00 & 10: 00 & 14: 00 & 18: 00 & 22: 00 & 02: 00 & 06: 00 & 10: 00 & 14: 00 & 18: 00 & 22: 00\end{array}$ 2017-09-12 (HH:MM)

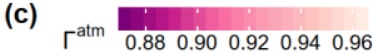

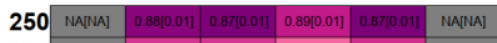

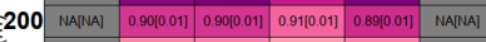

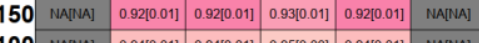

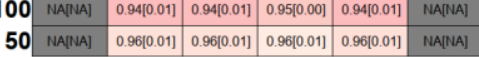

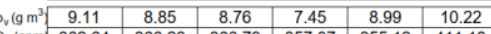
\begin{tabular}{c|c|c|c|c|c|} 
& \\
\hline
\end{tabular} \begin{tabular}{lllllll}
284.35 & 283.94 & 286.86 & 289.62 & 288.02 & 285.85 \\
\hline $\mathbf{0 2 : 0 0}$ & $\mathbf{0 6 : 0 0}$ & $10: 00$ & $14: 00$ & $18: 00$ & $22: 00$
\end{tabular} 2017-09-12 (HH:MM)

Figure S-11. As Figure S-6 but for 12 September 2017

(a)

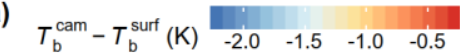

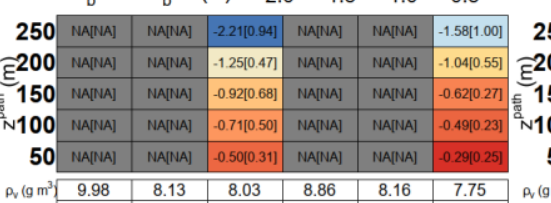

\begin{tabular}{c|c|c|c|c|c|c|}
$\rho_{v}(g \mathrm{~m}$ & 9.98 & 8.13 & 8.03 & 8.86 & 8.16 & 7.75 \\
\hline $\mathrm{O}_{2}(\mathrm{ppm}$ & 358.59 & 358.60 & 360.23 & 355.39 & 358.61 & 363.04 \\
\cline { 1 - 7 } & 79.42 & 75.00 & 05.81 & 39.75 & 07.18 & 72.00 \\
\hline
\end{tabular}

\begin{tabular}{c|c|c|c|c|c|c|}
$\mathrm{RH}(\%)$ & 79.42 & 75.00 & 65.81 & 69.75 & 67.18 & 72.00 \\
$T_{\mathrm{a}}(\mathrm{K})$ & 287.85 & 285.45 & 287.38 & 288.03 & 287.29 & 285.34 \\
\hline
\end{tabular}

02:00 06:00 10:00 14:00 18:00 22:00 2017-09-13 (HH:MM) (b) $L^{\mathrm{atm}}\left(\mathrm{W} \mathrm{m}^{-2} \mathrm{sr}^{-1}\right)$

$23 \quad 4$

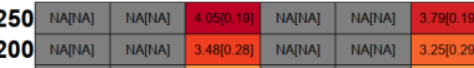

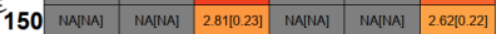

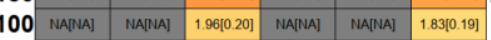

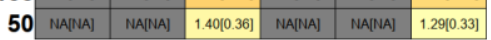

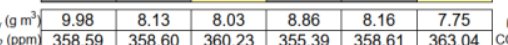
\begin{tabular}{c|c|c|c|c|c|c|}
$\mathrm{RH}(\%)$ & 79.42 & 35.60 & 36.23 & 355.39 & 358.61 & 363.04 \\
\hline
\end{tabular} $02: 00$ 06:00 10:00 14:00 18:00 22:00 2017-09-13 (HH:MM) (c)

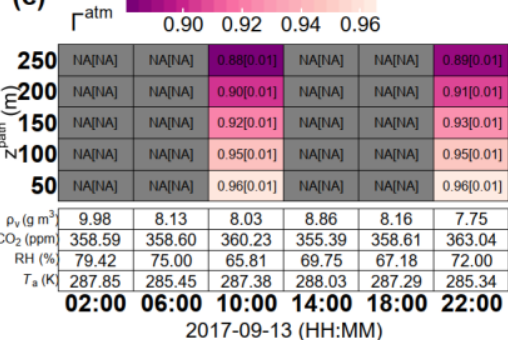

Figure S-12. As Figure S-6 but for 13 September 2017

(a)

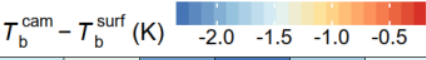

\begin{tabular}{ll|l|l|l|l|l|}
250 & $-1.55[1.02]$ & $-1.43[0.99]$ & $-2.08[0.70]$ & $-2.30[1.16]$ & $-1.77[1.16]$ & $-1.47[0.98]$ \\
\hline
\end{tabular}

\begin{tabular}{|l|l|l|l|l|l|l|l|}
\hline 200 & $-1.02[0.60]$ & $-1.07[0.51]$ & $-1.35[0.52]$ & $-1.76[0.54]$ & $-1.19[0.70]$ & $-0.95[0.63]$ \\
\hline
\end{tabular}

\begin{tabular}{llllllllll}
\hline 150 & $-0.590 .030]$ & $-0.60[0.26]$ & $-0.95[0.43]$ & $-1.21[0.48]$ & $-0.69[0.33]$ & $-0.55[0.32]$ \\
\hline
\end{tabular}

\begin{tabular}{lllllllll}
$\mathbf{N} 100$ & $-0.48[0.24]$ & $-0.51[0.24]$ & $-0.75[0.29]$ & $-0.90[0.32]$ & $-0.56[0.24]$ & $-0.45[0.23]$ \\
\hline
\end{tabular}

50

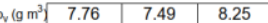

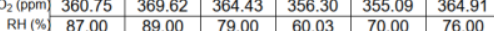

\begin{tabular}{|l|l|l|l|l|l|l|}
\hline 87.00 & 89.00 & 79.00 & 60.03 & 70.00 & 76.00 \\
\hline
\end{tabular}

$\begin{array}{lllllll}\mathbf{0 2}: 00 & 06: 00 & \mathbf{1 0}: 00 & \mathbf{1 4 : 0 0} & \mathbf{1 8 : 0 0} & \mathbf{2 2}: 00\end{array}$ 2017-09-14 (HH:MM) (b)

$L^{\mathrm{atm}}\left(\mathrm{W} \mathrm{m}^{-2} \mathrm{sr}^{-1}\right)$

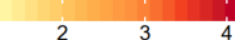

\begin{tabular}{ll|l|l|l|l|l|}
250 & $3.610 .18]$ & $3.46[0.14]$ & $3.96[0.20]$ & $4.05[0.19]$ & $3.92[0.19]$ & $3.73[0.19]$ \\
\hline
\end{tabular}

\begin{tabular}{|l|l|l|l|l|l|l|}
\hline 200 & $3.09[0.27]$ & $293[0.22]$ & $3.390 .30]$ & $3.48[0.30]$ & $3.38[0.30]$ & $3.19[0.28]$ \\
\hline
\end{tabular}

\begin{tabular}{|l|l|l|l|l|l|l|}
150 & $2.49[0.21]$ & $2.39[0.20]$ & $2.74[0.23]$ & $2.82[0.23]$ & $2.73[0.23]$ & $2.57[0.21]$ \\
\hline
\end{tabular}

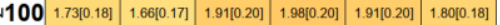
\begin{tabular}{|l|l|l|l|l|l|l|}
$\mathbf{5 0}$ & $1.22[0.32]$ & $1.16[0.30]$ & $1.35[0.35]$ & $1.41[0.36]$ & $1.35[0.35]$ & $1.27[0.33]$ \\
\hline
\end{tabular}

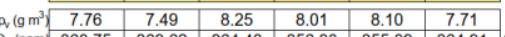

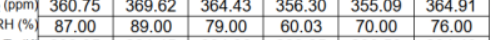
\begin{tabular}{lllllll}
282.35 & 281.45 & 284.86 & 288.85 & 286.50 & 284.40 \\
\hline $02: 00$ & $06: 00$ & $10: 00$ & $14: 00$ & $18: 00$ & $22: 00$
\end{tabular} 2017-09-14 (HH:MM) (c)

$\begin{array}{lllll}\Gamma^{\mathrm{atm}} & 0.90 & 0.92 & 0.94 & 0.96\end{array}$

Figure S-13. As Figure S-6 but for 14 September 2017

(a)

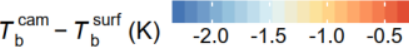

\begin{tabular}{ll|l|l|l|l|l|}
250 & $-1.48[1.03]$ & $-1.47[0.99]$ & $-2.15[0.57]$ & $-222[0.58]$ & $-229[1.08]$ & $-1.96[1.13]$ \\
\hline
\end{tabular}

\begin{tabular}{ll|l|l|l|l|l|l|l|}
\hline 200 & $-0.95[0.60]$ & $-0.96[0.58]$ & $-1.31[0.60]$ & $-1.47[0.56]$ & $-1.36[0.70]$ & $-1.25[0.65]$ \\
\hline
\end{tabular}

\begin{tabular}{llllllllll}
\hline & 150 & $-0.54[0.30]$ & $-0.55[0.28]$ & $-0.92[0.51]$ & $-1.01[0.55]$ & $-0.84[0.32]$ & $-0.75[0.30]$ \\
\hline
\end{tabular}

\begin{tabular}{lllllllll} 
& N100 & $-0.45[0.23]$ & $-0.45[0.23]$ & $-0.69[0.31]$ & $-0.83[0.32]$ & $-0.67[0.26]$ & $-0.59[0.25]$ \\
\hline
\end{tabular}

\begin{tabular}{|l|l|l|l|l|l|l|l|}
\hline $\mathbf{5 0}$ & $-0.22[0.25]$ & $-0.23[0.25]$ & $-0.43[0.29]$ & $-0.44[0.35]$ & $-0.39(0.31]$ & $-0.33[0.28]$ \\
\hline
\end{tabular}

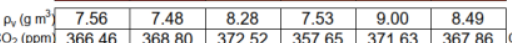

\begin{tabular}{c|c|c|c|c|c|c|}
\hline RH (\%) & 366.46 & 368.80 & 372.52 & 357.65 & 371.63 & 367.86 \\
\hline 84.25 & 88.00 & 79.00 & 61.68 & 83.00 & 86.00 \\
\hline
\end{tabular}

\begin{tabular}{ccccccc}
282.45 & 281.60 & 284.91 & 287.38 & 285.45 & 283.95 \\
\hline $02: 00$ & $06: 00$ & $10: 00$ & $14: 00$ & $18: 00$ & $22: 00$
\end{tabular} 2017-09-15 (HH:MM) (b) $L^{\mathrm{atm}}\left(\mathrm{W} \mathrm{m}{ }^{-2} \mathrm{sr}^{-1}\right)$

34

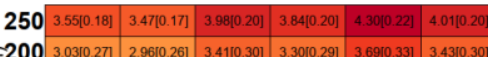

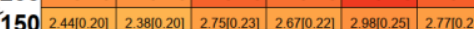

\begin{tabular}{|l|l|l|l|l|l|l|}
\hline $\mathbf{N} 100$ & $1.70[0.18]$ & $1.66[0.17]$ & $1.93[0.20]$ & $1.87[0.19]$ & $2.09[0.21]$ & $1.93[0.20]$ \\
\hline
\end{tabular} \begin{tabular}{|l|l|l|l|l|l|l|}
$\mathbf{5 0}$ & $1.20[0.31]$ & $1.17[0.30]$ & $1.36[0.35]$ & $1.32[0.34]$ & $1.48[0.38]$ & $1.36[0.35]$ \\
\hline
\end{tabular} \begin{tabular}{c|c|c|c|c|c|c|}
\hline$\rho_{1}\left(\mathrm{~g} \mathrm{~m}^{3}\right.$ & 7.56 & 7.48 & 8.28 & 7.53 & 9.00 & 8.49 \\
\cline { 2 - 7 } & &
\end{tabular}

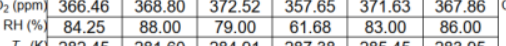
02:00 06:00 10:00 14:00 18:00 22:00 2017-09-15 (HH:MM)

250

\begin{tabular}{|l|l|l|l|l|l|l|}
\hline 200 & $0.91[0.01]$ & $0.91[0.01]$ & $0.90[0.01]$ & $0.90[0.01]$ & $0.90[0.01]$ & $0.91[0.01]$ \\
\hline
\end{tabular}

\begin{tabular}{|l|l|l|l|l|l|l|l|}
\hline 150 & $0.93[0.01]$ & $0.93[0.01]$ & $0.92[0.01]$ & $0.92[0.01]$ & $0.92[0.01]$ & $0.93[0.01$ \\
\hline
\end{tabular}

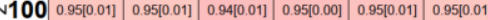
\begin{tabular}{|l|l|l|l|l|l|l|}
50 & $0.96[0.01]$ & $0.97[0.01]$ & $0.96[0.01]$ & $0.96[0.01]$ & $0.96[0.01]$ & $0.96[0.01]$ \\
\hline & 7.7 & 7.49 & 8.25 & 8.01 & 8.10 & 7.71 \\
\hline
\end{tabular}

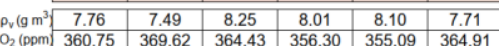
\begin{tabular}{ll|l|l|l|l|l|}
\hline $\mathbf{R}$ & $\mathbf{8}(\% .00$ & 89.00 & 79.00 & 60.03 & 70.00 & 76.00 \\
\hline
\end{tabular} \begin{tabular}{|llllll}
\hline $\mathbf{2} 2: 00$ & $06: 00$ & $10: 00$ & $14: 00$ & $18: 00$ & $22: 00$
\end{tabular} 2017-09-14 (HH:MM)

(c) $\Gamma^{\mathrm{atm}} 0.8750 .900 \quad 0.9250 .950$

\begin{tabular}{|l|l|l|l|l|l|l|}
$\mathbf{2 5 0}$ & $0.890 .01]$ & $0.89[0.011$ & $0.88[0.011$ & $0.89[0.011$ & 0.8710011 & 0.8890 .01 \\
\hline
\end{tabular} \begin{tabular}{lll|l|l|l|l|l|}
150 & $0.93[0.01]$ & $0.93[0.01]$ & $0.92[0.01]$ & $0.93[0.01]$ & $0.92[0.01]$ & $0.92[0.01]$
\end{tabular}

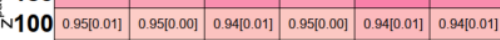
\begin{tabular}{ll|l|l|l|l|l|l|}
50 & $0.96[0.01]$ & $0.96[0.01]$ & $0.96[0.01]$ & $0.96[0.01]$ & $0.96[0.01]$ & $0.96[0.01]$
\end{tabular}

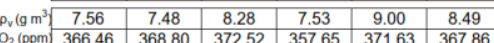
\begin{tabular}{c|c|c|c|c|c|c|c|}
$\mathrm{CO}_{2}$ (ppm & 766.46 & 368.80 & 372.52 & 357.65 & 371.63 & 367.86 \\
\hline $\mathrm{RH}(\%)$ & 84.25 & 88.00 & 79.00 & 61.68 & 83.00 & 86.00 \\
\hline
\end{tabular} 02:00 06:00 10:00 14:00 18:00 22:00 2017-09-15 (HH:MM)

Figure S-14. As Figure S-6 but for 15 September 2017

(a)

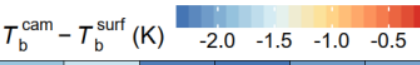

$250-1.84[1.23]-1.60[1.17]$

\begin{tabular}{llllllll}
200 & $-1.10[0.71]$ & $-1.02[0.64]$ & $-1.45[0.63]$ & $-1.68[0.64]$ & $-1.30[0.68]$ & $-1.28[0.68]$ \\
\hline
\end{tabular}

\begin{tabular}{lllllllll}
\hline & 150 & $-0.61[0.33]$ & $-0.56[0.32]$ & $-0.97[0.40]$ & $-1.09[0.51]$ & $-0.80[0.30]$ & $-0.76[0.31]$ \\
\hline
\end{tabular}

$\begin{array}{lllllllll} & \text { N100 } & -0.50[0.26] & -0.47[0.25] & -0.76[0.25] & -0.83[0.28] & -0.63[0.23] & -0.62[0.25]\end{array}$

50

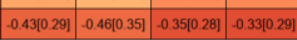

(b)

(b) $L^{\text {atm }}\left(\mathrm{W} \mathrm{m}^{-2} \mathrm{sr}^{-1}\right)$

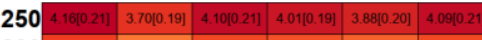

\begin{tabular}{|l|l|l|l|l|l|l|}
\hline 200 & $3.57[0.31]$ & $3.17[0.28]$ & $3.51[0.31]$ & $3.44[0.30]$ & $3.34[0.29]$ & $3.50[0.31]$ \\
\hline
\end{tabular}

\begin{tabular}{|l|l|l|l|l|l|l|}
150 & $288[0.24]$ & $2.55[0.21]$ & $2.83[0.23]$ & $2.78[0.23]$ & $2.69[0.22]$ & $2.82[0.23$ \\
\hline
\end{tabular}

\begin{tabular}{llllllll}
\hline $2.01[0.21]$ & $1.770 .18]$ & $1.98[0.20]$ & $1.95[0.20]$ & $1.890 .19]$ & 1.9710 .20 \\
\hline
\end{tabular} \begin{tabular}{ll|l|l|l|l|l|l|}
$\mathbf{5 0}$ & $1.42[0.37]$ & $1.25[0.32]$ & $1.40[0.36]$ & $1.38[0.36]$ & $1.33[0.34]$ & $1.39[0.36]$ \\
\hline
\end{tabular}

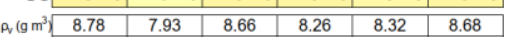

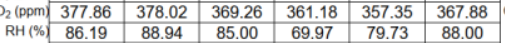

\begin{tabular}{|c|c|c|c|c|c|}
\hline 284.45 & 282.35 & 284.45 & 286.83 & 284.83 & 283.95 \\
\hline $\mathbf{0 2}: 00$ & $\mathbf{0 6 : 0 0}$ & $\mathbf{1 0} 00$ & $\mathbf{1 4 : 0 0}$ & $\mathbf{1 8 : 0 0}$ & $\mathbf{2 2 : 0 0}$
\end{tabular} 2017-09-16 (HH:MM) $\begin{array}{lllllll}\text { (c) } & \Gamma^{\mathrm{atm}} & 0.88 & 0.90 & 0.92 & 0.94 & 0.96\end{array}$

\begin{tabular}{|r|r|r|r|r|r|r|}
$\mathbf{2 5 0}$ & $0.88[0.011$ & $0.89[0.01]$ & $0.88[0.01]$ & $0.88[0.01]$ & $0.88[0.01]$ & $0.88[0.01$ \\
\hline $\mathbf{2 0 0}$ & $0.90[0.011$ & $0.90[0.01]$ & $0.90[0.01]$ & $0.90[0.01]$ & $0.90[0.01]$ & $0.90[0.01]$
\end{tabular}

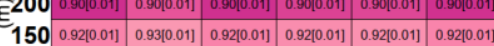

$\begin{array}{lllllllll} & \text { N100 } & 0.94[0.01] & 0.95[0.01] & 0.94[0.01] & 0.94[0.00] & 0.95[0.01] & 0.94[0.011\end{array}$ \begin{tabular}{|l|l|l|l|l|l|l|}
50 & $0.96[0.01]$ & $0.96[0.01]$ & $0.96[0.01]$ & $0.96[0.01]$ & $0.96[0.01]$ & $0.96[0.01]$ \\
\hline
\end{tabular}

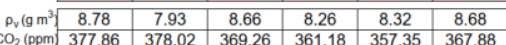

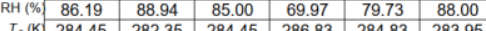
\begin{tabular}{llllll}
\hline $\mathbf{2 8 2} .45$ & 282.35 & 284.45 & 286.83 & 284.83 & 283.95 \\
\hline $26: 00$ & $10: 00$ & $14: 00$ & $18: 00$ & $22: 00$
\end{tabular} 2017-09-16 (HH:MM)

Figure S-15. As Figure S-6 but for 16 September 2017 
(a) $\begin{array}{lllll}T_{\mathrm{b}}^{\text {cam }}-T_{\mathrm{b}}^{\text {surf }}(\mathrm{K})-2.5 & -2.0 & -1.5 & -1.0 & -0.5\end{array}$ $250-2.091 .25]-2.141 .200]-2461.02]-254[1.49]-215[1.32]-1.82[1.37]$ \begin{tabular}{|l|l|l|l|l|l|l|}
\hline $\mathbf{2} 200$ & $-1.29[0.66]$ & $-1.34[0.65]$ & $-1.59[0.69]$ & $-1.83[0.77]$ & $-1.38[0.75]$ & $-1.16[0.74]$ \\
\hline
\end{tabular}

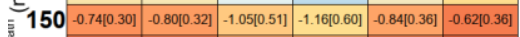

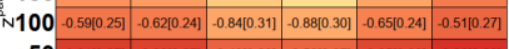
50

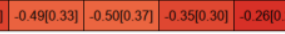

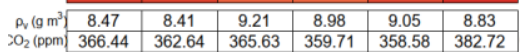

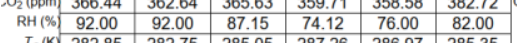

02:00 06:00 10:00 14:00 18:00 22:00 2017-09-17 (HH:MM) (b) $L^{\text {atm }}\left(\mathrm{W} \mathrm{m}^{-2} \mathrm{sr}^{-1}\right) \quad 2 \quad 3 \quad 4$

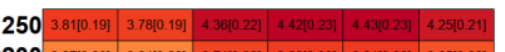

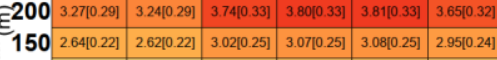

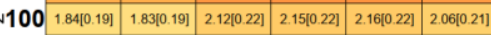

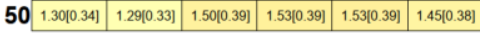

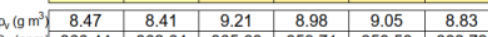

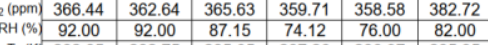
$02: 00$ 06:00 10:00 $14: 00$ 18:00 22:00 2017-09-17 (HH:MM) (c)

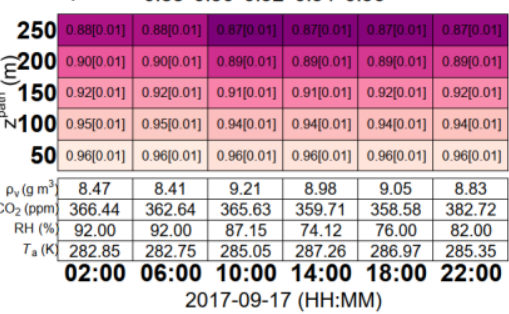

Figure S-16. As Figure S-6 but for 17 September 2017

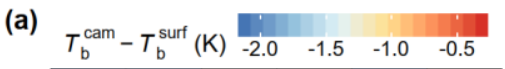

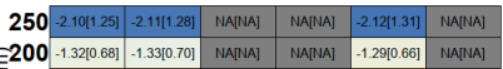

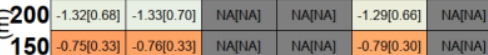

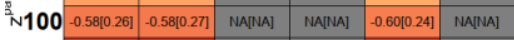
50

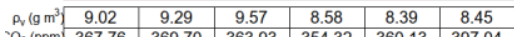

\begin{tabular}{llllllll}
$\mathrm{O}_{2}(\mathrm{pom})$ & 367.76 & 369.70 & 363.93 & 354.32 & 360.13 & 397.04 \\
\hline
\end{tabular}

\begin{tabular}{c|ccccccc}
$R H(k)$ & 92.00 & 94.00 & 85.41 & 72.28 & 71.00 & 89.33 \\
$T_{a}(K)$ & 283.85 & 283.97 & 285.99 & 286.93 & 286.85 & 283.27 \\
\hline
\end{tabular}

02:00 06:00 10:00 14:00 18:00 22:00 2017-09-18 (HH:MM) (b) $L^{\mathrm{atm}}\left(\mathrm{W} \mathrm{m}^{-2} \mathrm{sr}^{-1}\right) 1.52 .02 .53 .03 .54 .0$

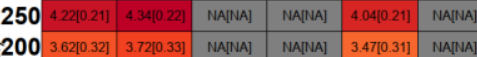

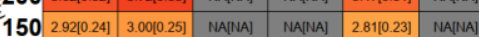

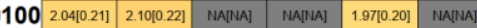

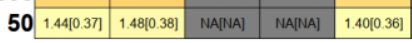

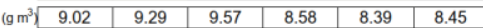
\begin{tabular}{|lllllll}
367.76 & 369.70 & 363.93 & 354.32 & 360.13 & 397.04 \\
\hline
\end{tabular}

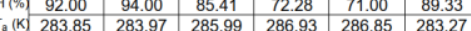
$\begin{array}{lllllll}283.85 & 283.97 & 285.99 & 286.93 & 286.85 & 283.27 \\ 02: 00 & \mathbf{0 6}: 00 & 14: 00 & 18: 00 & 22: 00\end{array}$ 2017-09-18 (HH:MM) (c)

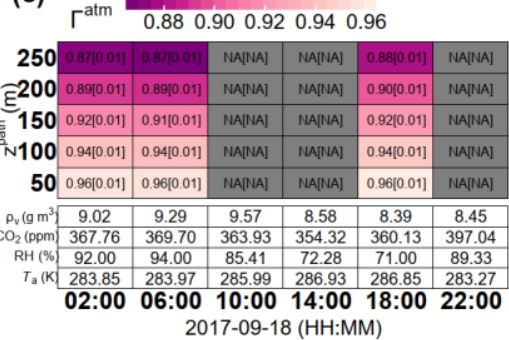

Figure S-17. As Figure S-6 but for 18 September 2017

(a)

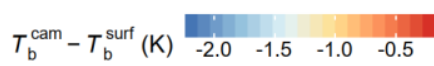

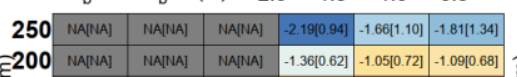

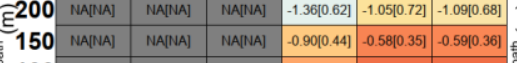

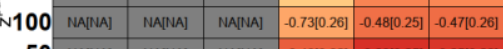

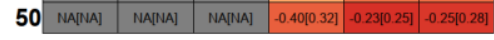

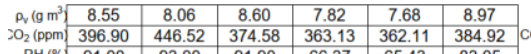

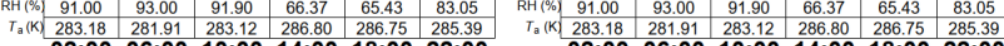

02:00 06:00 10:00 14:00 18:00 22:00 2017-09-19 (HH:MM) (b)

$L^{\mathrm{atm}}\left(\mathrm{W} \mathrm{m}^{-2} \mathrm{sr}^{-1}\right)$

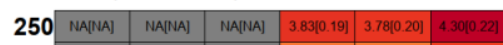

200 MANAN

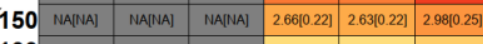

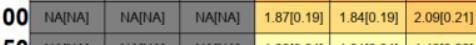

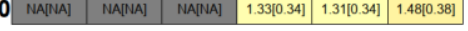
02:00 06:00 10:00 14:00 18:00 22:00 2017-09-19 (HH:MM)

Figure S-18. As Figure S-6 but for 19 September 2017
(a)
$T_{\mathrm{b}}^{\text {cam }}-T_{\mathrm{b}}^{\text {surf }}(\mathrm{K}) \quad \begin{array}{llll}\mathrm{a} & -3 & -2 & -1\end{array}$

250 -208[1.56] $-2.16[1.43]-3.05[1.44]-3.44[214]-245[1.57]-2541.54]$

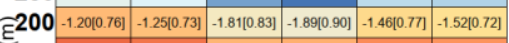

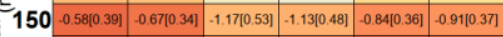

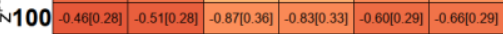

(b) $L^{\mathrm{atm}}\left(\mathrm{W} \mathrm{m}^{-2} \mathrm{sr}^{-1}\right) \quad 2 \quad 3 \quad 4 \quad 5$

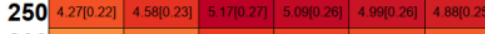

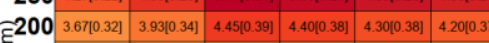

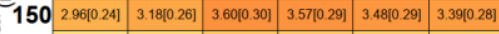

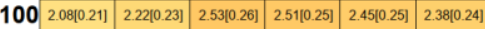
\begin{tabular}{|l|l|l|l|l|l|l|}
50 & $1.470 .38]$ & $1.57[0.40]$ & $1.80[0.46]$ & $1.79[0.46]$ & $1.74[0.45]$ & $1.69[0.43]$ \\
\hline
\end{tabular}

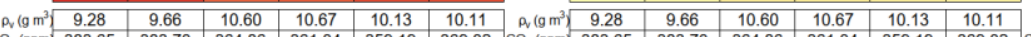

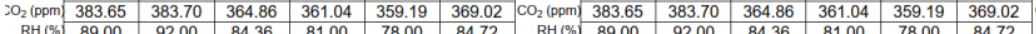

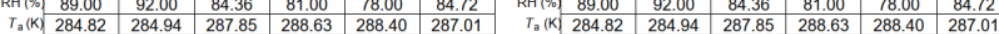
$\begin{array}{llllllllllll}02: 00 & 06: 00 & 10: 00 & 14: 00 & 18: 00 & 22: 00 & 02: 00 & 06: 00 & 10: 00 & 14: 00 & 18: 00 & 22: 00\end{array}$ 2017-09-20 (HH:MM)

(c) $\Gamma^{\mathrm{atm}} 0.880 .90 \quad 0.920 .940 .96$

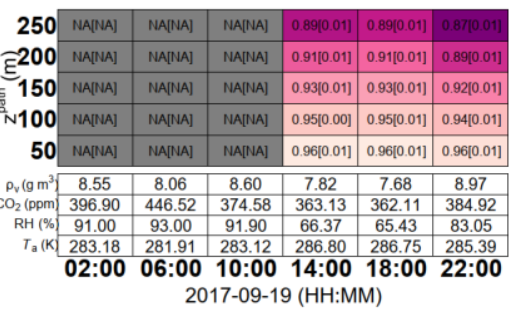

(c)

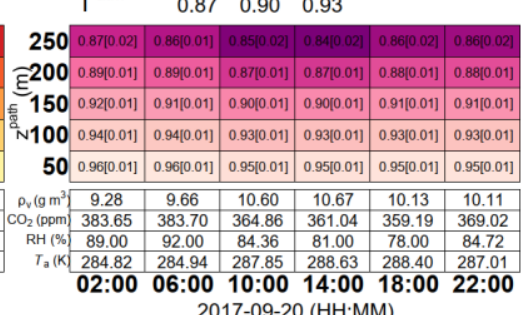

Figure S-19. As Figure S-6 but for 20 September 2017

(a) $T_{\mathrm{b}}^{\text {cam }}-T_{\mathrm{b}}^{\text {surf }}(\mathrm{K})-3 \quad-2 \quad-1$

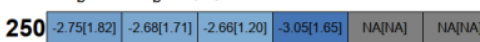

\begin{tabular}{|c|c|c|c|c|c|c|}
\hline 200 & $-1.590 .79]$ & $-1.56[0.80]$ & $-1.6110 .68]$ & $-1.81[0.85]$ & NAINA] & NA[NA] \\
\hline
\end{tabular}

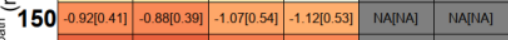

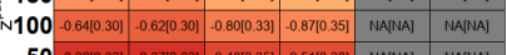

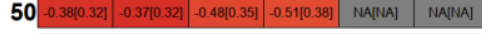

\begin{tabular}{|c|c|c|c|c|c|c|c|c|c|c|c|c|}
$\mathrm{p}_{2}(\mathrm{gm})$ & 10.69 & 10.65 & 10.05 & 10.51 & 11.71 & 9.93 \\
\hline
\end{tabular}

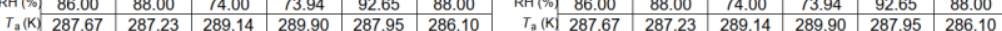
02:00 06:00 10:00 14:00 18:00 22:00 2017-09-21 (HH:MM) (b) $L^{\text {atm }}\left(\mathrm{W} \mathrm{m}^{-2} \mathrm{sr}^{-1}\right) \quad 2 \quad 3 \quad 4 \quad 5$

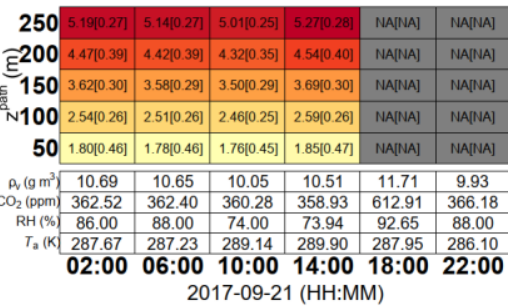
2017-09-21 (HH:MM) (c)

$\Gamma^{\mathrm{atm}} 0.8500 .8750 .9000 .9250 .950$

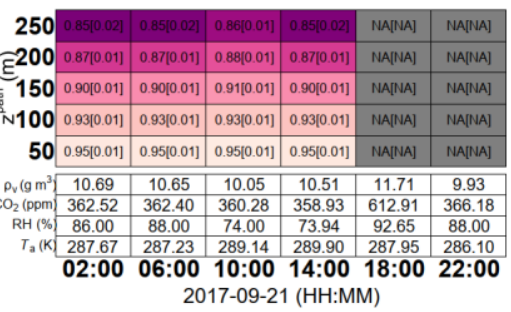

Figure S-20. As Figure S-6 but for 21 September 2017 
(a)

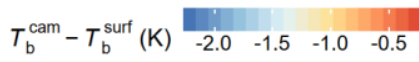

\begin{tabular}{ll|l|l|l|l|l|l|}
250 & $-1.81[1.38]$ & $-1.56[1.40]$ & $-204[1.31]$ & $-221[1.18]$ & $-2.17[1.45]$ & $-2.10[1.4$ \\
\hline
\end{tabular} \begin{tabular}{|l|l|l|l|l|l|l|l|}
\hline & 200 & $-1.28[0.63]$ & $-1.06[0.66]$ & $-1.12[0.48]$ & $-1.54[0.75]$ & $-1.32[0.73]$ & $-1.28[0.68]$ \\
\hline
\end{tabular}

\begin{tabular}{lllllllll}
\hline & 150 & $-0.66[0.34]$ & $-0.49[0.35]$ & $-0.78[0.68]$ & $-0.98[0.62]$ & $-0.76[0.34]$ & $-0.72[0.35]$ \\
\hline
\end{tabular}

\begin{tabular}{llllllll|l} 
& $\mathrm{N} 100$ & $-0.55[0.28]$ & $-0.44[0.28]$ & $-0.63[0.47]$ & $-0.78[0.37]$ & $-0.57[0.27]$ & $-0.54[0.27]$ \\
\hline
\end{tabular}

50

(b) $L^{\mathrm{atm}}\left(\mathrm{W} \mathrm{m}^{-2} \mathrm{sr}^{-1}\right) 1.52 .02 .53 .03 .54 .04 .5$ \begin{tabular}{|r|r|l|l|l|l|l|}
$\mathbf{2 5 0}$ & $\mathbf{4 2 5 [ 0 . 1 9 ]}$ & $4.10[0.17]$ & $4.00[0.17]$ & $428[0.21]$ & $4.48[0.23]$ & $451[0.23]$ \\
\hline $\mathbf{2 0 0}$ & $3.62[0.27]$ & $3.490 .26]$ & $3.410 .23]$ & $3.67[0.32]$ & $3.86[0.34]$ & $3.88[0.34]$ \\
\hline
\end{tabular} \begin{tabular}{|c|c|c|c|c|c|c|}
\hline $\mathbf{2 0 0}$ & $3.62[0.27]$ & $3.490 .26]$ & $3.41[0.23]$ & $3.67[0.32]$ & $3.86[0.34]$ & $3.88[0.34]$ \\
\hline $\mathbf{1 5 0}$ & $2.96[0.25]$ & $285[0.24]$ & $279[0.23]$ & $297[0.24]$ & $3.13[0.26]$ & $314[0.26]$ \\
\hline
\end{tabular} \begin{tabular}{|l|l|l|l|l|l|l|l} 
& $N 100$ & $2.07[0.21]$ & $1.99[0.21]$ & $1.95[0.20]$ & $2.09[0.21]$ & $2.2010 .22]$ & $220[0.22]$ \\
\hline
\end{tabular} \begin{tabular}{|l|l|l|l|l|l|l|}
$\mathbf{5 0}$ & $1.46[0.38]$ & $1.40[0.36]$ & $1.39[0.35]$ & $1.49[0.38]$ & $1.56[0.40]$ & $1.56[0.40]$ \\
\hline
\end{tabular}

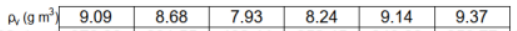

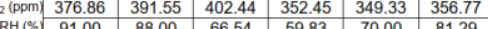
\begin{tabular}{c|c|c|c|c|c|c|c|}
\hline $\mathrm{RH}(\%)$ & 91.00 & 88.00 & 66.54 & 59.83 & 70.00 & 81.29 \\
\hline$T_{\mathrm{a}}(\mathrm{K})$ & 284.15 & 283.95 & 286.99 & 289.37 & 288.47 & 286.45 \\
\cline { 2 - 9 }
\end{tabular} 02:00 06:00 10:00 14:00 18:00 22:00 2017-09-22 (HH:MM)

\begin{tabular}{|l|l|l|l|l|l}
\hline & 9.09 & 8.62 & 7.93 & 8.24 & \\
\hline
\end{tabular}

\begin{tabular}{c|c|c|c|c|c|c|}
$\mathrm{RH}(\%)$ & 91.00 & 88.00 & 66.54 & 59.83 & 70.00 & 81.29 \\
$T_{a}(\mathrm{~K})$ & 284.15 & 283.05 & 286.99 & 289.37 & 288.47 & 286.45 \\
\hline
\end{tabular} \begin{tabular}{lllllll}
284.150 & 283.95 & 286.99 & 289.37 & 288.4 & 286.45 \\
\hline
\end{tabular} 2017-09-22 (HH:MM) (c)

$\Gamma^{\mathrm{atm}} \quad 0.88 \quad 0.900 .920 .940 .96$

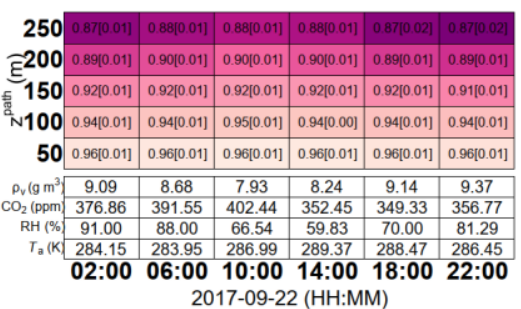

Figure S-21. As Figure S-6 but for 22 September 2017

(a)

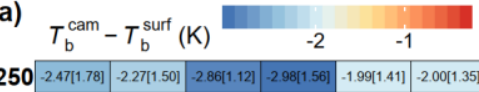

\begin{tabular}{|l|l|l|l|l|l|l|}
\hline 200 & $-1.46[0.71]$ & $-1.38[0.71]$ & $-1.71[0.69]$ & $-1.80[0.79]$ & $-1.22[0.72]$ & $-1.23[0.70]$ \\
\hline
\end{tabular}

\begin{tabular}{|l|l|l|l|l|l|l|l|}
\hline & 150 & $-0.80[0.33]$ & $-0.81[0.36]$ & $-1.13[0.57]$ & $-1.11[0.55]$ & $-0.69[0.35]$ & $-0.68[0.36]$ \\
\hline
\end{tabular}

\begin{tabular}{ll|l|l|l|l|l|l|}
\hline N100 & $-0.57[0.27]$ & $-0.58[0.28]$ & $-0.85[0.39]$ & $-0.88[0.35]$ & $-0.54[0.26]$ & $-0.53[0.27]$ \\
\hline
\end{tabular}

\begin{tabular}{|l|l|l|l|l|l|l|}
\hline $\mathbf{5 0}$ & $-0.33[0.29]$ & $-0.34[0.30]$ & $-0.55[0.36]$ & $-0.50[0.39]$ & $-0.31[0.28]$ & $-0.31[0.29$ \\
\hline
\end{tabular}

\begin{tabular}{c|c|c|c|c|c|c|}
$\rho_{v}\left(\mathrm{gm}^{3}\right.$ & 9.72 & 9.73 & 10.29 & 9.39 & 8.87 & 9.07 \\
$\mathrm{CO}_{2}(\mathrm{ppm})$ & 358.08 & 359.66 & 366.45 & 349.96 & 350.08 & 373.33 \\
\cline { 2 - 7 }
\end{tabular}

\begin{tabular}{c|c|c|c|c|c|c|} 
RH (\%) & 82.95 & 79.00 & 75.95 & 63.37 & 65.00 & 74.90 \\
\cline { 2 - 8 } & 28671 & 287.53 & 28909 & 290.59 & 2922 & 287.25 \\
\hline
\end{tabular}

02:00 06:00 $10: 00 \quad 14: 00 \quad 18: 00 \quad 22: 00$ 2017-09-23 (HH:MM)

(b)

$L^{\mathrm{atm}}\left(\mathrm{W} \mathrm{m}^{-2} \mathrm{sr}^{-1}\right)$

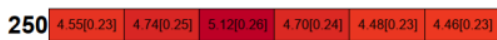

\begin{tabular}{|l|l|l|l|l|l|l|l|}
\hline $\mathbf{2} 200$ & $3.92[0.35]$ & $4.08[0.36]$ & $4.42[0.35]$ & $4.06[0.36]$ & $3.86[0.34]$ & $3.83[0.34]$ \\
\hline
\end{tabular}

\begin{tabular}{|c|c|c|c|c|c|c|}
\hline 150 & $3.17[0.26]$ & $3.30[0.27]$ & $3.58[0.29]$ & $3.30[0.27]$ & $3.13[0.26]$ & $3.10[0.26]$ \\
\hline
\end{tabular}

\begin{tabular}{|l|l|l|l|l|l|l|}
$\mathbf{N} 100$ & $223[0.23]$ & $2.32[0.24]$ & $252[0.25]$ & $233[0.23]$ & $220[0.22]$ & $217[0.22]$ \\
\hline
\end{tabular}

\begin{tabular}{|l|l|l|l|l|l|l|}
\hline $\mathbf{5 0}$ & $1.58[0.41]$ & $1.65[0.42]$ & $1.80[0.46]$ & $1.66[0.42]$ & $1.57[0.40]$ & $1.54[0.40]$ \\
\hline
\end{tabular}

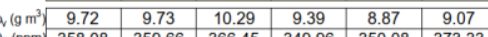
\begin{tabular}{c|c|c|c|c|c|c|}
$\mathrm{RH}(\%)$ & 82.08 & 359.66 & 366.45 & 349.96 & 350.08 & 373.33 \\
\hline & 82.95 & 79.00 & 75.95 & 63.37 & 65.00 & 74.90 \\
\hline
\end{tabular} 02:00 06:00 10:00 14:00 18:00 22:00 2017-09-23 (HH:MM)

Figure S-22. As Figure S-6 but for 23 September 2017

(a)

$$
T_{\mathrm{b}}^{\mathrm{cam}}-T_{\mathrm{b}}^{\text {surf }}(\mathrm{K}) \quad-3 \quad-2 \quad-1
$$

\begin{tabular}{ll|l|l|l|l|l|}
250 & $-2.41[1.66]$ & $-2.34[1.93]$ & $-364[0.91]$ & $-2.58[1.91]$ & $-2.45[1.54]$ \\
\hline
\end{tabular}

\begin{tabular}{|l|l|l|l|l|l|l|}
\hline है $^{200}$ & $-1.39[0.79]$ & $-1.30[0.85]$ & $-1.68[0.83]$ & $-1.97[0.84]$ & $-1.43[0.77]$ & $-2.01[0.97]$ \\
\hline
\end{tabular}

\begin{tabular}{ll|l|l|l|l|l|l|}
\hline & 150 & $-0.74[0.38]$ & $-0.61[0.44]$ & $-1.10[0.87]$ & $-1.22[0.74]$ & $-0.86[0.34]$ & $-1.07[0.45]$ \\
\hline
\end{tabular}

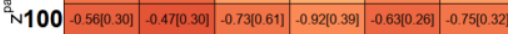

50

\begin{tabular}{l|l|l|l|l|l|l|}
$0.9 \mathrm{~m}^{3}$ & 10.22 & 10.10 & 10.44 & 9.22 & 9.44 & 11.72 \\
\hline
\end{tabular}

\begin{tabular}{l|l|l|l|l|l|l}
$\mathrm{CO}_{2}(\mathrm{ppm})$ & 393.72 & 376.41 & 369.43 & 354.90 & 356.94 & 384.68 \\
\hline
\end{tabular}

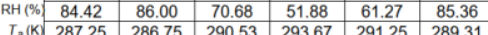

.(k) \begin{tabular}{|c|c|c|c|c|c|}
28.77 .25 & 286.75 & 290.53 & 293.67 & 291.25 & 289.31 \\
\hline 02.00 & 06.00 & $10: 00$ & 14.00 & 18.00 & 22.00
\end{tabular} 2017-09-24 (HH:MM) (b)

$$
L^{\mathrm{atm}}\left(\mathrm{W} \mathrm{m}^{-2} \mathrm{sr}^{-1}\right) \quad 2 \quad 3 \quad 4 \quad 5
$$

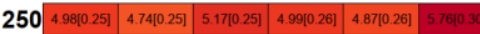

\begin{tabular}{|c|c|c|c|c|c|c|c|}
\hline 200 & $4290.38]$ & $4.09[0.36]$ & $4.48[0.35]$ & $4.30[0.37]$ & $4.20[0.37]$ & $4.98[0.44]$ \\
\hline
\end{tabular}

\begin{tabular}{|l|l|l|l|l|l|l|}
\hline 150 & $3.47[0.28]$ & $3.31[0.27]$ & $3.64[0.30]$ & $3.50[0.29]$ & $3.41[0.28]$ & $4.04[0.33]$ \\
\hline
\end{tabular}

\begin{tabular}{llllll|l|l|l|} 
& $N 100$ & $2.43[0.25]$ & $2.33[0.24]$ & $256[0.26]$ & $247[0.25]$ & $2.41[0.24]$ & $2.84[0.29]$ \\
\hline
\end{tabular} \begin{tabular}{rl|l|l|l|l|l|l|}
\hline $\mathbf{5 0}$ & $1.72[0.44]$ & $1.65[0.42]$ & $1.84[0.46]$ & $1.77[0.45]$ & $1.72[0.44]$ & $2.02[0.52]$ \\
\hline
\end{tabular}

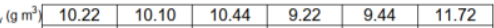
\begin{tabular}{c|c|c|c|c|c|c|}
\hline $\mathrm{RH}(\%)$ & 393.72 & 376.41 & 369.43 & 354.90 & 356.94 & 384.68 \\
\hline & 84.42 & 00.00 & 70.00 & 51.08 & 01.27 & 85.36 \\
\hline
\end{tabular}

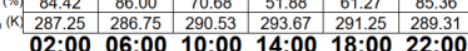
2017-09-24 (HH:MM) (c) $\Gamma^{\mathrm{atm}} \quad 0.8750 .9000 .9250 .950$

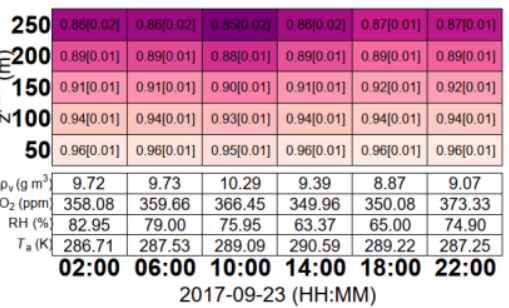

(c) $\Gamma^{\mathrm{atm}} \quad 0.850 .8750 .90 \oplus .925 .950$

\begin{tabular}{|l|l|l|l|l|l|l|l|}
$\mathbf{2 5 0}$ & $0.85[0.02]$ & $0.86[0.02]$ & $0.85[0.02]$ & $0.87[0.02]$ & $0.86[0.02]$ & $0.83[0.03$ \\
\hline $\mathbf{2 0 0}$ & $0.88[0.01]$ & $0.88[0.01]$ & $0.88[0.01]$ & $0.890 .01]$ & $0.89[0.01]$ & $0.86[0.01$ \\
\hline
\end{tabular}

\begin{tabular}{|l|l|l|l|l|l|l|l|l}
150 & $0.91[0.01]$ & $0.91[0.01]$ & $0.90[0.01]$ & $0.91[0.01]$ & $0.91[0.01]$ & 0.890 .011 \\
\hline
\end{tabular}

\begin{tabular}{lllllllll}
100 & $0.93[0.01]$ & $0.94[0.01]$ & $0.93[0.01]$ & $0.94[0.01]$ & $0.94[0.01]$ & $0.92[0.01]$ \\
\hline
\end{tabular} \begin{tabular}{ll|l|l|l|l|l|l|}
$\mathbf{5 0}$ & $0.95[0.01]$ & $0.95[0.01]$ & $0.95[0.01]$ & $0.96[0.01]$ & $0.96[0.01]$ & $0.95[0.01]$ \\
\hline
\end{tabular} $\rho_{v}\left(\mathrm{~g} \mathrm{~m}^{3} \begin{array}{|c|c|c|c|c|c|}\hline 10.22 & 10.10 & 10.44 & 9.22 & 9.44 & 11.72 \\ \hline\end{array}\right.$ \begin{tabular}{|c|c|c|c|c|c|}
\hline (ppm) 393.72 & 376.41 & 369.43 & 354.90 & 356.94 & 384.68 \\
\hline 04.42 & 86.00 & 70.68 & 51.88 & 61.27 & 85.36 \\
\hline
\end{tabular} \begin{tabular}{c|c|c|c|c|c|c|}
\hline $\mathrm{RH}(\%)$ & 84.42 & 86.00 & 70.68 & 51.88 & 61.27 & 85.36 \\
\hline$T_{a}(\mathrm{~K})$ & 287.25 & 286.75 & 290.53 & 293.67 & 291.25 & 289.31 \\
& 02.00 & $06: 00$ & 10.00 & 14.00 & $18: 00$ & 22.00
\end{tabular} 02:00 $06: 00 \quad 10: 00$ 14:00 18:00 22:00 2017-09-24 (HH:MM)

Figure S-23. As Figure S-6 but for 24 September 2017 (same as Figure 7 in main text)

(a)

$T_{\mathrm{b}}^{\mathrm{cam}}-T_{\mathrm{b}}^{\text {surf }}(\mathrm{K}) \quad-4 \quad \begin{array}{llll}1 & -3 & -2 & -1\end{array}$

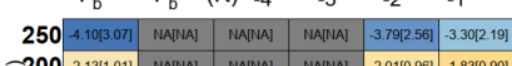

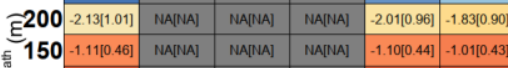

\begin{tabular}{l|l|l|l|l|l|l|l|l}
\hline N100 & $-0.75[0.32]$ & NAINA] & NAINA] & NAINA] & $-0.77[0.32]$ & $-0.72[0.31]$ \\
\hline
\end{tabular}

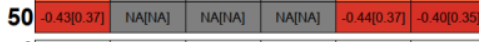

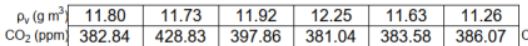

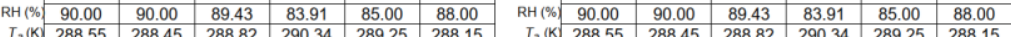

02:00 06:00 10:00 14:00 18:00 22:00 2017-09-25 (HH:MM)

(b) $L^{\text {atm }}\left(\mathrm{W} \mathrm{m}^{-2} \mathrm{sr}^{-1}\right) 2 \quad 3 \quad 4 \quad 5$

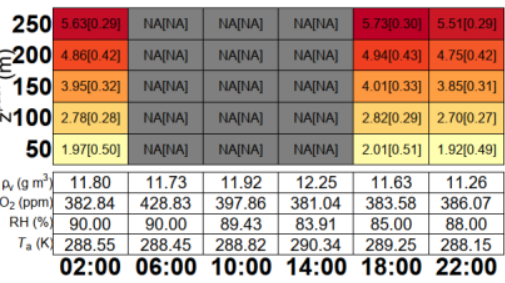
2017-09-25 (HH:MM)

Figure S-24. As Figure S-6 but for 25 September 2017

(a)

$$
T_{\mathrm{b}}^{\text {cam }}-T_{\mathrm{b}}^{\text {surf }}(\mathrm{K}) \quad-4 \quad-3 \quad-2 \quad-1
$$

\begin{tabular}{ll|l|l|l|l|l|}
250 & $-3.08[2.31]$ & $-2.93[1.99]$ & $-4.36[1.52]$ & $-3.94[3.03]$ & $-2.96[2.03]$ & $-3.35[240]$ \\
\hline
\end{tabular} \begin{tabular}{ll|l|l|l|l|l|l|}
\hline 200 & $-1.64[0.94]$ & $-1.69[0.89]$ & $-2.11[1.00]$ & $-2.07[1.10]$ & $-1.56[0.90]$ & $-1.77[0.97]$ \\
\hline
\end{tabular}

\begin{tabular}{lllllllll} 
& 150 & $-0.82[0.45]$ & $-0.90[0.43]$ & $-1.28[0.64]$ & $-1.13[0.64]$ & $-0.80[0.44]$ & $-0.86[0.46]$ \\
\hline
\end{tabular}

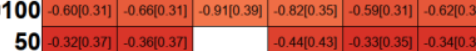

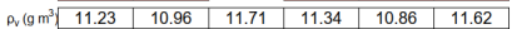

\begin{tabular}{|l|l|l|l|l|l|}
\hline & 11.30 \\
\hline
\end{tabular}

\begin{tabular}{lllll|l|l|l|l|l|l|l|l|l|}
\hline $\mathrm{CO}_{2}$ (ppm) & 382.98 & 398.61 & 401.89 & 380.73 & 386.30 & 404.93 & $\mathrm{CO}_{2}$ (ppm) 382.98 & 398.61 & 401.89 & 380.73 & 386.30 & 404.93 \\
\hline
\end{tabular}

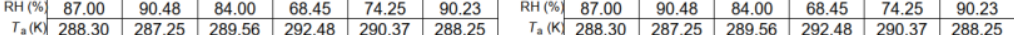
02:00 $06: 00$ 10:00 $14: 00 \quad 18: 00 \quad 22: 00$ 2017-09-26 (HH:MM) (b)

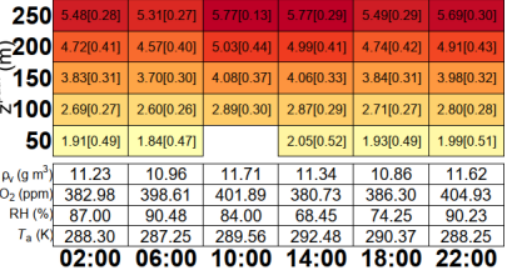
2017-09-26 (HH:MM) (c) $\Gamma^{\mathrm{atm}} 0.8250 .850 .8750 .900 .925$

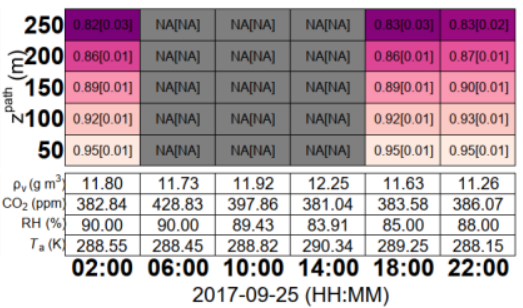

(c) $\Gamma^{\mathrm{atm}} 0.825 .850 .87 \$ .90 \oplus .925 .950$

\begin{tabular}{|l|l|l|l|l|l|l|l|}
$\mathbf{2 5 0}$ & $0.84[0.02]$ & $0.84[0.02]$ & $0.82[0.031$ & $0.83[0.03]$ & $0.84[0.02]$ & 0.8350 .03 \\
\hline $\mathbf{2 0 0}$ & $0.87[0.01]$ & $0.87[0.01]$ & $0.86[0.01]$ & $0.86[0.01]$ & $0.87[0.01]$ & $0.86[0.01]$ \\
\hline
\end{tabular}

\begin{tabular}{c|c|c|c|c|c|c|c|c|}
150 & $0.90[0.01]$ & $0.90[0.01]$ & $0.89[0.01]$ & $0.90[0.01]$ & $0.90[0.01]$ & $0.89[0.01]$ \\
\hline
\end{tabular}

$\begin{array}{llllllll}100 & 0.93[0.01] & 0.93[0.01] & 0.92[0.01] & 0.93[0.01] & 0.93[0.01] & 0.92[0.01]\end{array}$

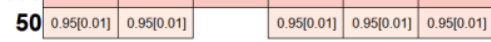

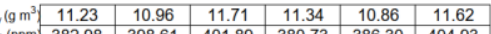

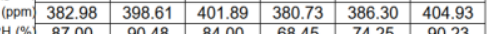

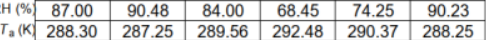
02:00 $06: 00$ 10:00 14:00 18:00 22:00 2017-09-26 (HH:MM)

Figure S-25. As Figure S-6 but for 26 September 2017 


\section{S5. Time series images of atmosphere and emissivity correction}

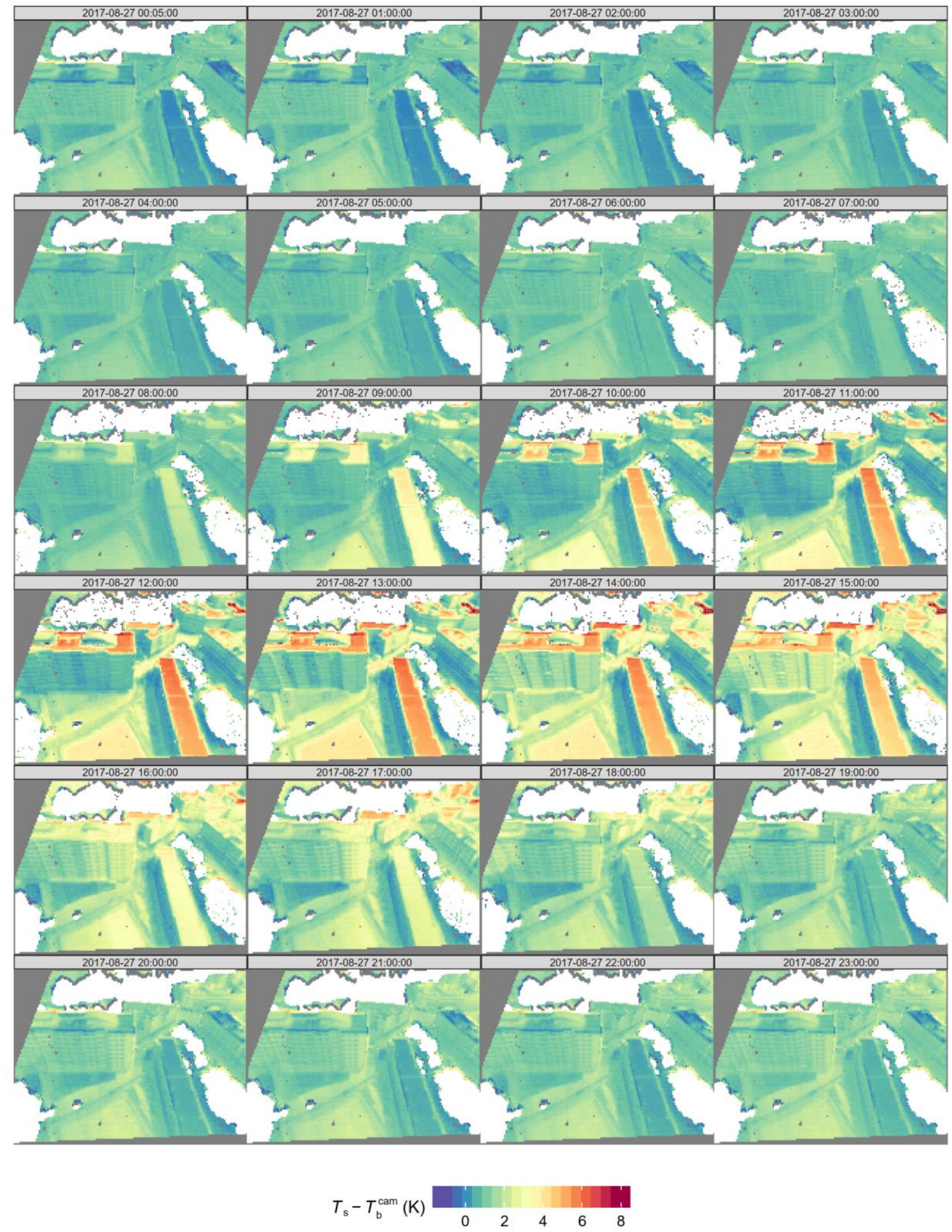

Figure S-26. Difference between at sensor-radiance as brightness temperature $\left(T_{b}{ }^{c a m}\right)(K)$ and corrected surface temperature $\left(T_{s}\right)$ accounting for atmosphere and emissivity effects for camera C1. Each image is the instantaneous value of $T_{s}-T_{b}^{\text {cam }}(K)$ using emissivity of 0.93 for all non-vegetative surfaces. See Section 3 for details on the correction. 

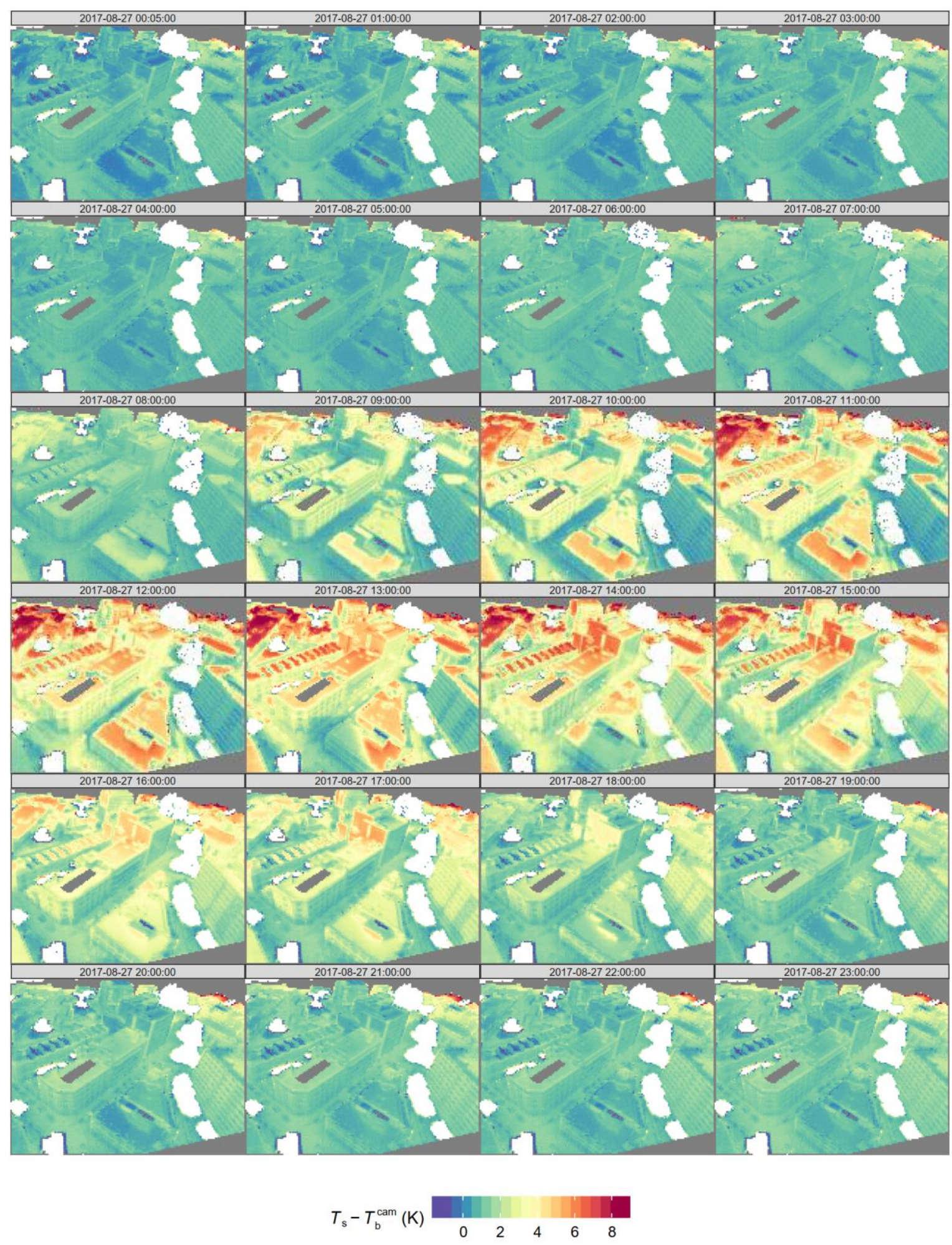

Figure S-27. As Figure S-26 but for C2. 
Morrison et al. https://doi.org/10.1016/j.rse.2019.111524

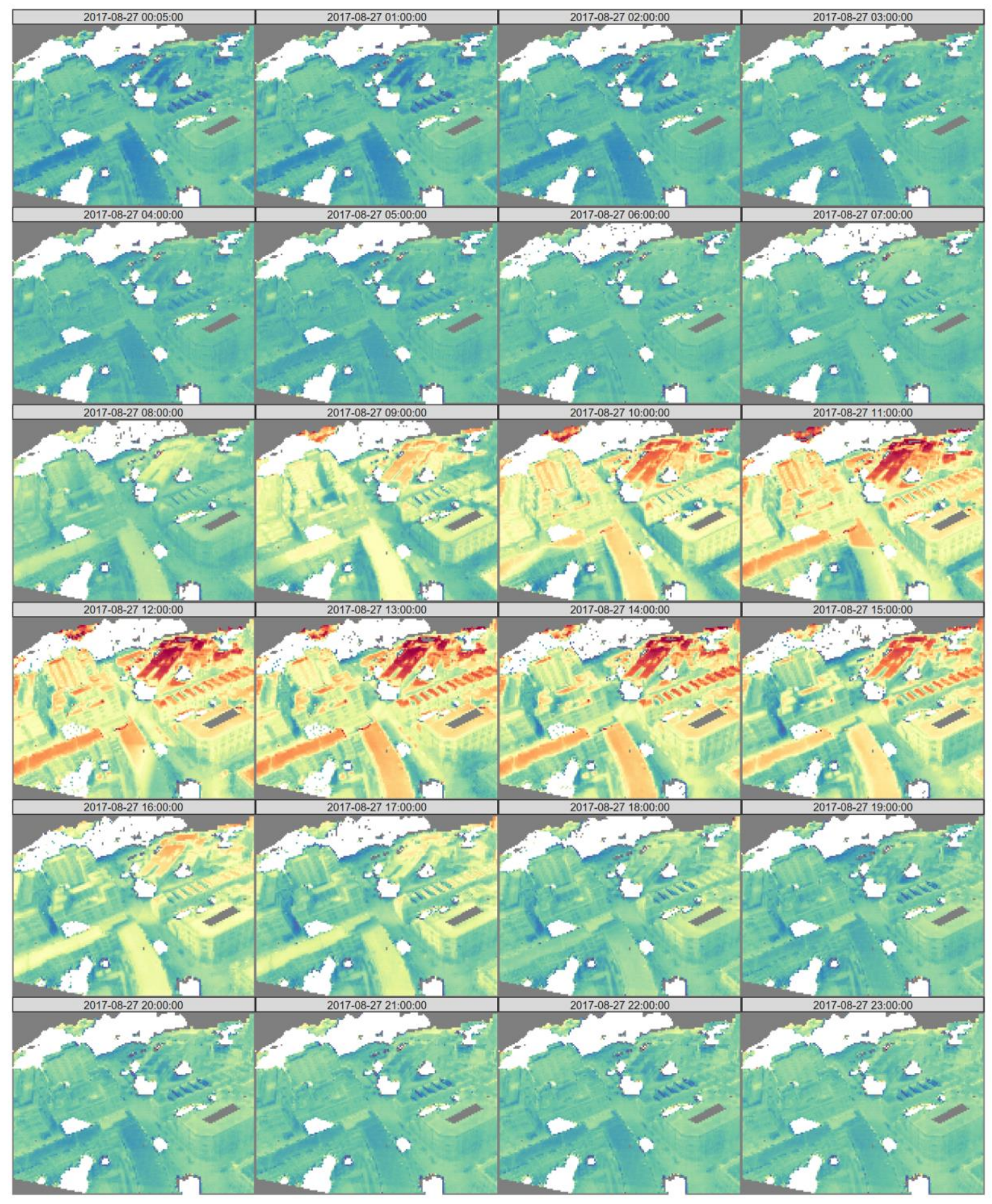

$$
\begin{array}{llllll}
T_{\mathrm{s}}-T_{\mathrm{b}}^{\mathrm{cam}}(\mathrm{K}) & 0 & 2 & 4 & 6 & 8
\end{array}
$$

Figure S-28. As Figure S-26 but for C3. 
Morrison et al. https://doi.org/10.1016/j.rse.2019.111524

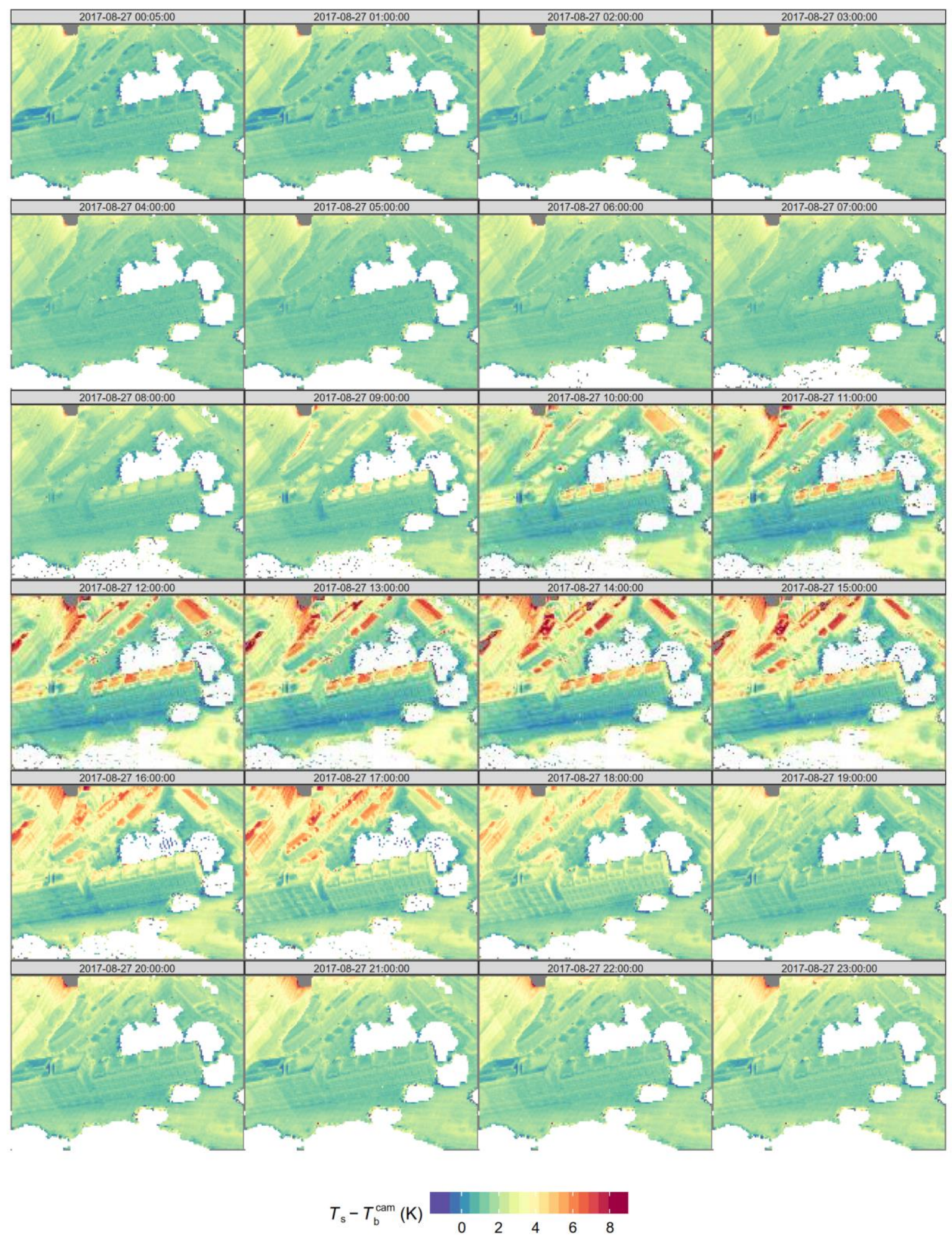

Figure S-29. As Figure S-26 but for C4. 
Morrison et al. https://doi.org/10.1016/j.rse.2019.111524

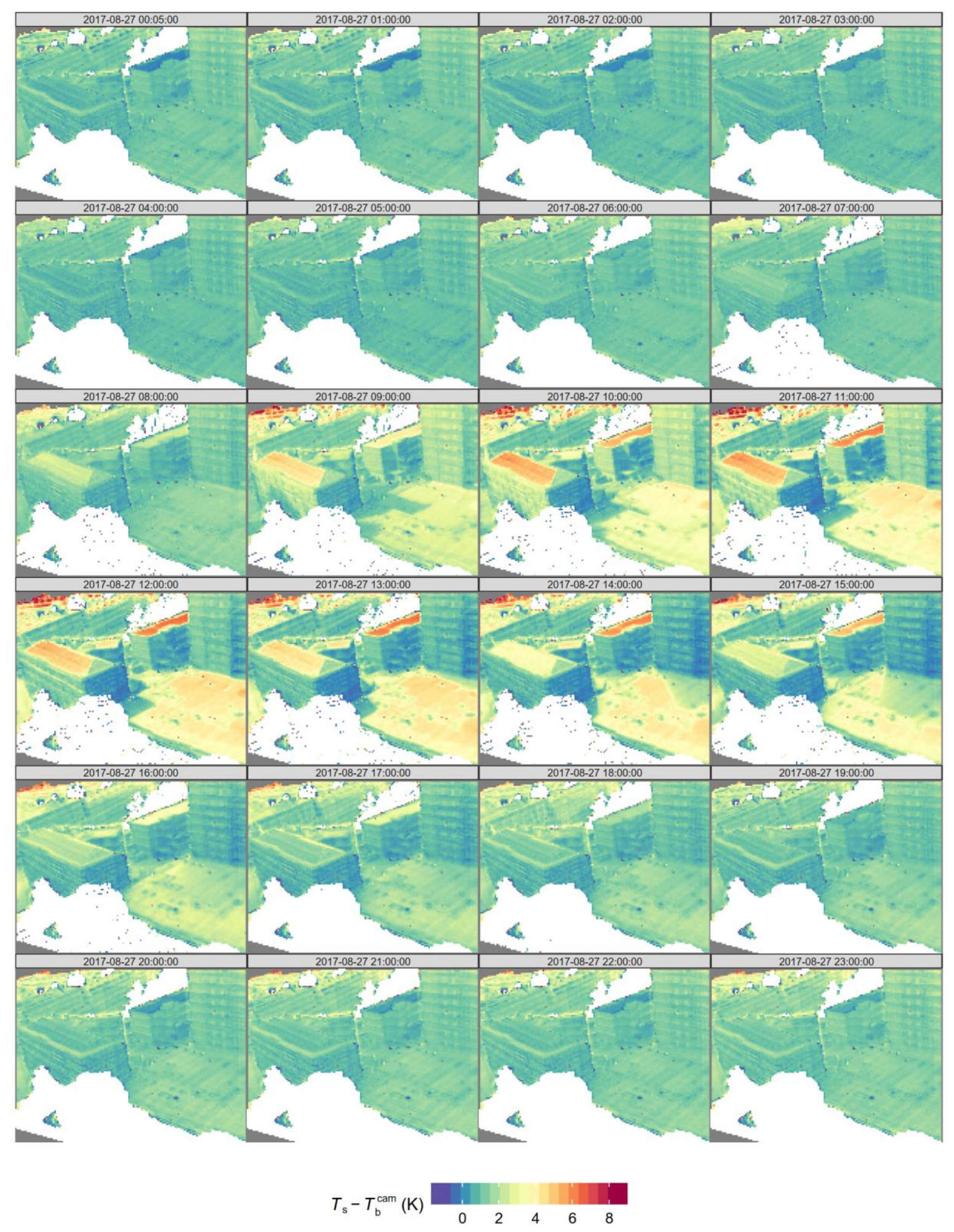

Figure S-30. As Figure S-26 but for C5. 
Morrison et al. https://doi.org/10.1016/..rse.2019.111524

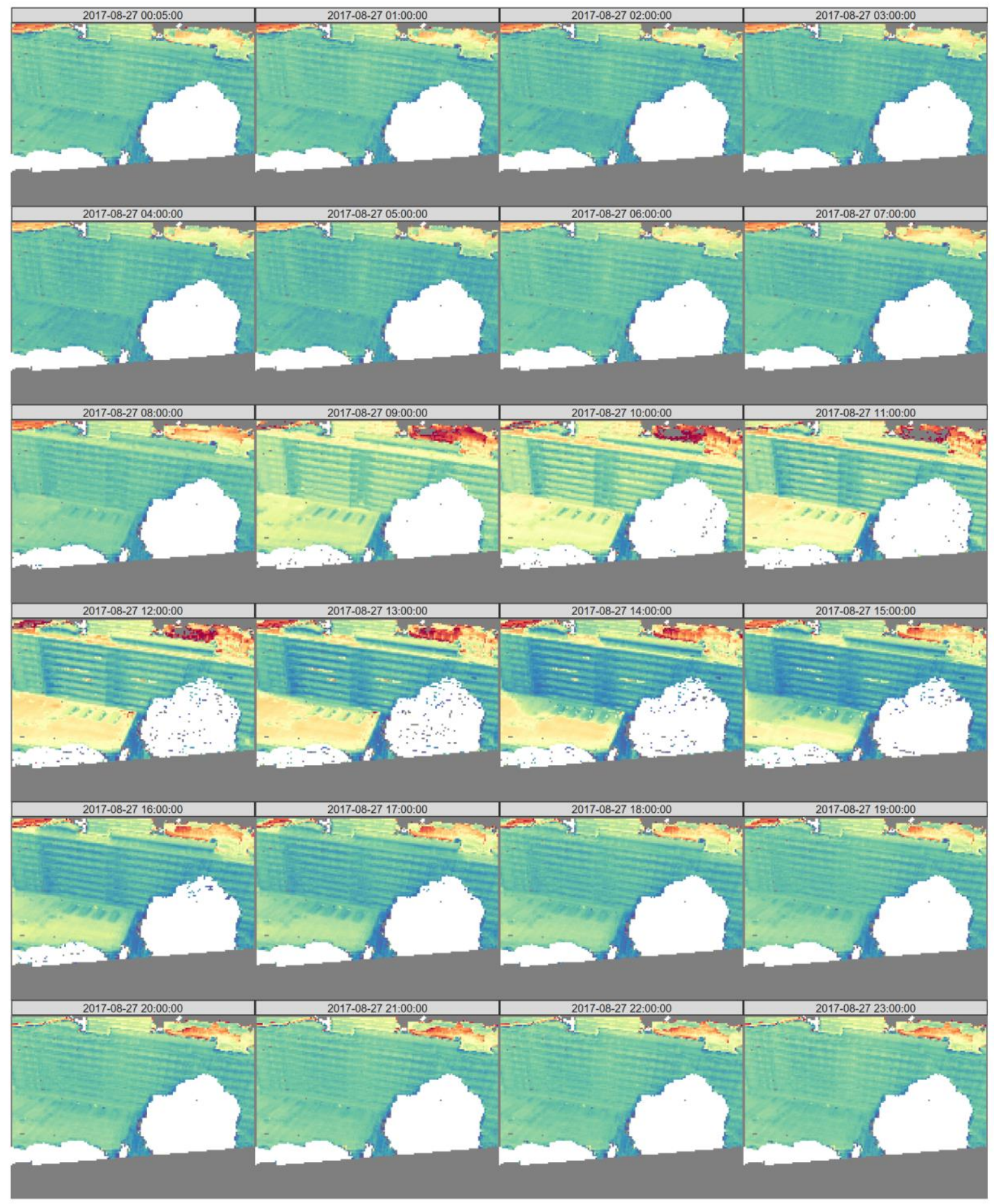

$$
\begin{array}{lllllll}
T_{\mathrm{s}}-T_{\mathrm{b}}^{\mathrm{cam}}(\mathrm{K}) & 0 & 2 & 4 & 6 & 8
\end{array}
$$

Figure S-31. As Figure S-26 but for C6. 